\title{
Off-Equilibrium Speed Control of a Multistage Molecular Rotor: Twofold Chemical Fueling by Acid or Silver(I)
}

\author{
Abir Goswami, Suchismita Saha, Emad Elramadi, Amit Ghosh, and Michael Schmittel,* \\ Center of Micro- and Nanochemistry and Engineering, University of Siegen \\ Adolf-Reichwein-Str. 2, D-57068 Siegen, Germany. \\ Organische Chemie I, \\ E-mail: $\underline{\text { schmittel@chemie.uni-siegen.de }}$ \\ Fax: (+49) 271-740-3270
}

\section{Table of Contents}

1. Synthesis. S2-S5

2. Synthesis and characterization of complexes........................S5-S11

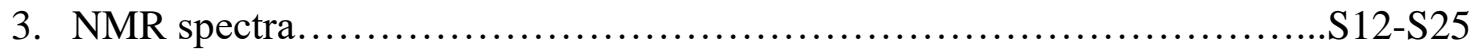

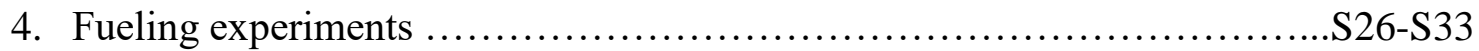

5. Variable temperature ${ }^{1} \mathrm{H} \mathrm{NMR},{ }^{1} \mathrm{H}-{ }^{1} \mathrm{H}$ ROESY and determination of kinetic parameters

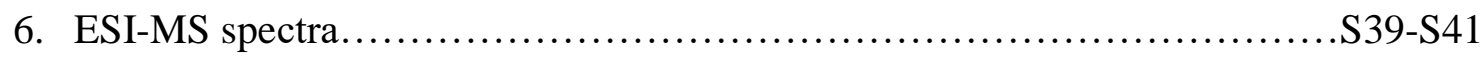

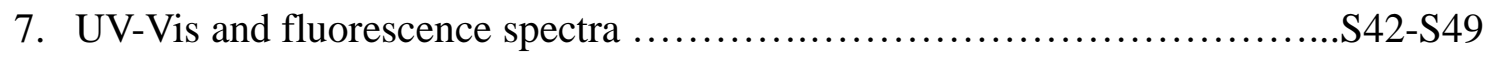

8. Binding constant measurements.................................. S49-S50

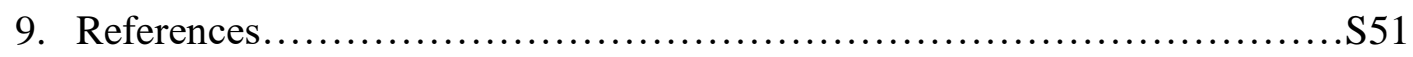




\section{Synthesis}

\section{General Remarks}

Commercial reagents $(4,5,7,8,14)$ were used without any further purification, while compounds $10,{ }^{1} \mathbf{1 1},{ }^{2} 12,{ }^{3} \mathbf{1 3},{ }^{3} \mathbf{1 5}^{3}$ and $16^{4}$ were synthesized according to literature known procedures. All solvents were distilled prior to use. All rotor preparations were performed directly in the NMR tube using $\mathrm{CD}_{2} \mathrm{Cl}_{2}$ as solvent.

${ }^{1} \mathrm{H}$ and ${ }^{13} \mathrm{C}$ NMR spectra were measured on Bruker Avance (400 MHz), Jeol ECZ (500 MHz) and Varian VNMR-S $600(600 \mathrm{MHz})$ spectrometers using a deuterated solvent as the lock and residual protiated solvent as internal reference $\left(\mathrm{CDCl}_{3}: \delta_{\mathrm{H}} 7.26 \mathrm{ppm}, \delta_{\mathrm{C}} 77.0 \mathrm{ppm}\right.$; $\left.\mathrm{CD}_{2} \mathrm{Cl}_{2}: \delta_{\mathrm{H}} 5.32 \mathrm{ppm}, \delta_{\mathrm{C}} 53.8 \mathrm{ppm}\right)$.

The following abbreviations are used to define NMR peak pattern: $\mathrm{s}=$ singlet, $\mathrm{d}=$ doublet, $\mathrm{t}$ $=$ triplet, $\mathrm{dd}=$ doublet of doublets, $\mathrm{ddd}=$ doublet of doublets of doublets, $\mathrm{td}=$ triplet of doublets, $b r=$ broad, $m=$ multiplet. Coupling constant values are given in Hertz $(\mathrm{Hz})$ and, wherever possible, assignment of protons is provided. The numbering of different carbons in different molecular skeletons does not necessarily follow IUPAC nomenclature rules; it is exclusively implemented for assigning NMR signals.

All electrospray ionization (ESI-MS) spectra were recorded on a Thermo-Quest LCQ deca and theoretical isotopic distributions of the mass signals were calculated using IsoPro 3.0 software. Melting points of compounds were measured on a BÜCHI 510 instrument and are not corrected. Infrared spectra were recorded on a Perkin Elmer Spectrum Two FT-IR instrument. Elemental analysis was performed using the EA-3000 CHNS analyzer. UV-vis spectra were recorded on a Cary $100 \mathrm{UV}$-vis $(298 \mathrm{~K})$ spectrometer in quartz cuvettes. Binding constants were determined through UV-vis titrations in combination with a 1:1 binding formula of two ligands or with SPECFIT/32TM global analysis system by Spectrum Software Associates (Marlborough, MA). Column chromatography was performed either on silica gel (60-400 mesh) or neutral alumina (Fluka, 0.05-0.15 mm, Brockmann Activity 1). Merck silica gel (60 F254) or neutral alumina (150 F254) sheets were used for thin layer chromatography (TLC). 


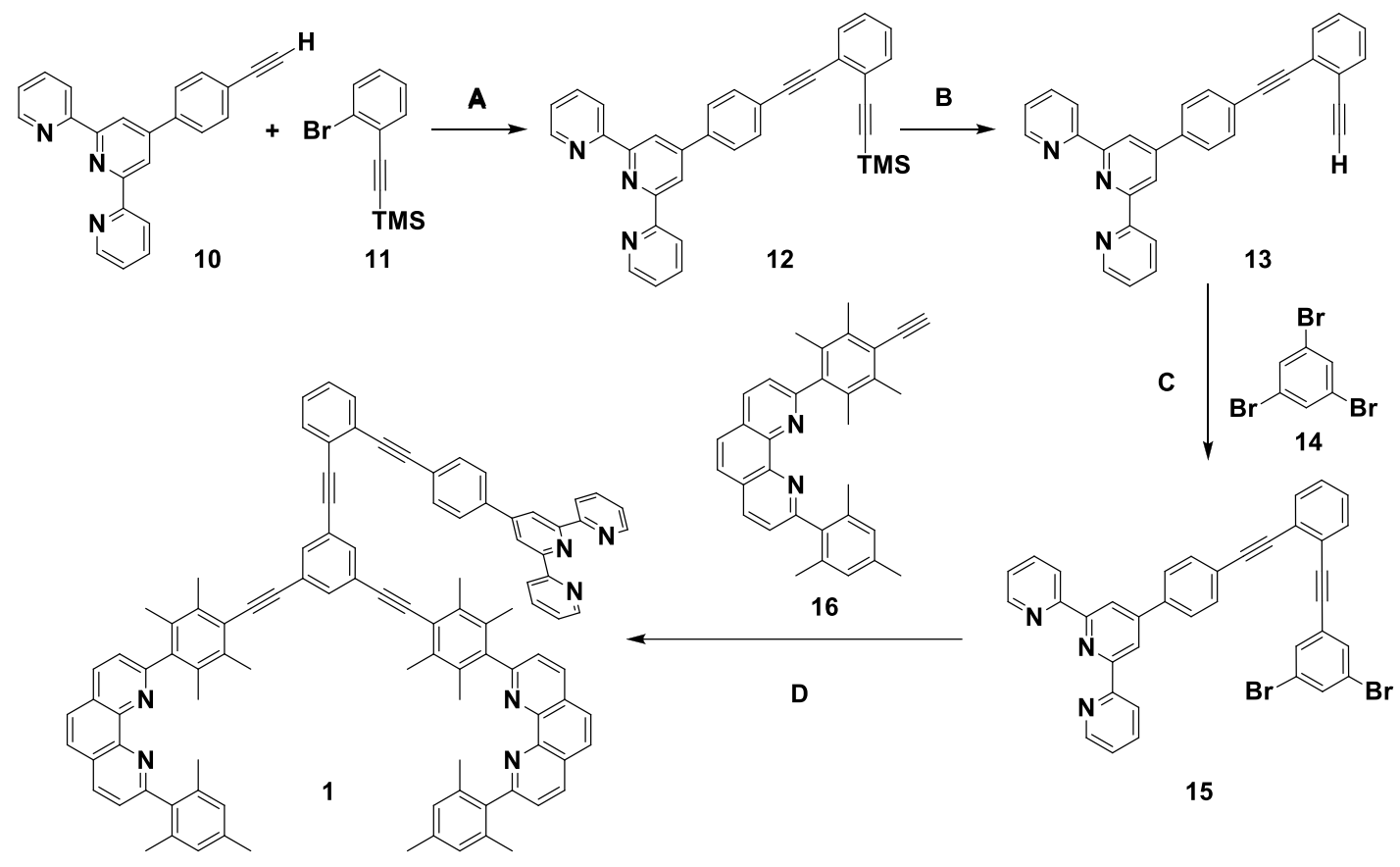

Scheme 1. The preparation of ligand 1 was executed as described earlier. ${ }^{3}$ Reaction conditions: (A) $\mathrm{Pd}\left(\mathrm{PPh}_{3}\right)_{4}, \mathrm{DMF} / \mathrm{Et}_{3} \mathrm{~N}, 80{ }^{\circ} \mathrm{C}, 15 \mathrm{~h}, 53 \%$. (B) $\mathrm{K}_{2} \mathrm{CO}_{3}, \mathrm{THF} / \mathrm{MeOH}, \mathrm{rt}, 3 \mathrm{~h}, 95 \%$. (C) Compound 14, $\mathrm{Pd}\left(\mathrm{PPh}_{3}\right)_{4}, \mathrm{DMF} / \mathrm{Et}_{3} \mathrm{~N}, 70{ }^{\circ} \mathrm{C}, 12 \mathrm{~h}, 58 \%$. (D) Compound 16, $\mathrm{Pd}\left(\mathrm{PPh}_{3}\right)_{4}$, $\mathrm{DMF} / \mathrm{Et}_{3} \mathrm{~N}, 70{ }^{\circ} \mathrm{C}, 20 \mathrm{~h}, 78 \%$.

\section{Spectroscopic data for ligand $1 .^{3}$}

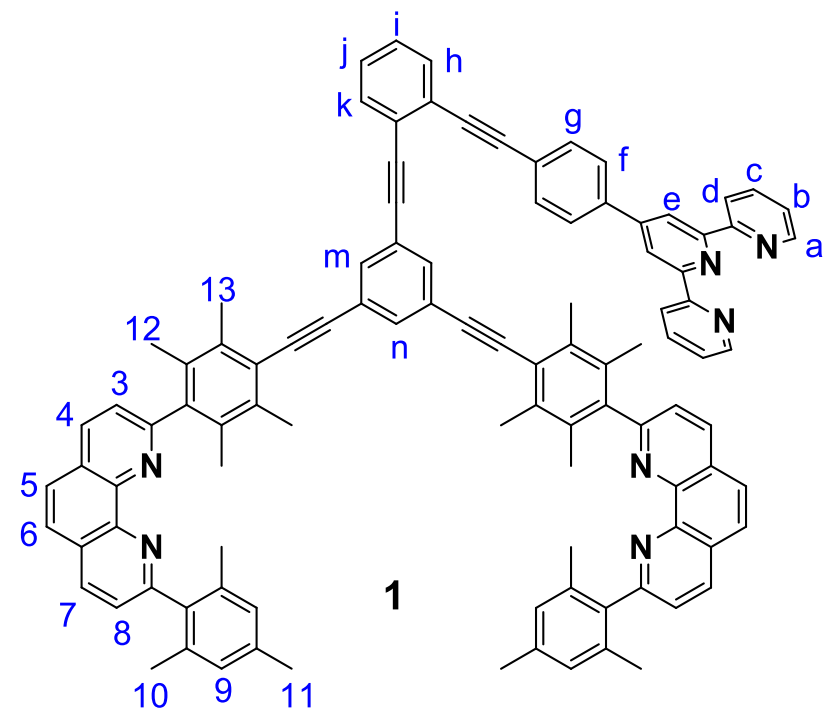

Mp. > $250{ }^{\circ} \mathrm{C}$. IR (KBr): $\tilde{v}=663,827,845,758,792,853,1035,1249,1384,1468,1493$, 1514, 1567, 1585, 2027, 2157, 2219, 2310, 2853, 2924, $2957 \mathrm{~cm}^{-1}$. ${ }^{\mathbf{1}} \mathbf{H}-\mathbf{N M R}$ (400 MHz, CD2 $\left.\mathbf{C l}_{2}\right): \delta=1.82(\mathrm{~s}, 12 \mathrm{H}, 13-\mathrm{H}), 2.04(\mathrm{~s}, 12 \mathrm{H}, 10-\mathrm{H}), 2.32$ (s, 6H, 11-H), 2.51 (s, 12H, 12- 
H), $6.94(\mathrm{~s}, 4 \mathrm{H}, 9-\mathrm{H}), 7.28\left(\mathrm{ddd},{ }^{3} \mathrm{~J}=7.6,{ }^{3} \mathrm{~J}=4.8 \mathrm{~Hz},{ }^{4} \mathrm{~J}=1.2 \mathrm{~Hz}, 2 \mathrm{H}, \mathrm{b}-\mathrm{H}\right), 7.40-7.43$ (m, $2 \mathrm{H},[\mathrm{i}+\mathrm{j}]-\mathrm{H}), 7.44\left(\mathrm{~d},{ }^{3} \mathrm{~J}=8.2 \mathrm{~Hz}, 2 \mathrm{H}, 8 / 3-\mathrm{H}\right), 7.55\left(\mathrm{~d},{ }^{3} J=8.2 \mathrm{~Hz}, 2 \mathrm{H}, 3 / 8-\mathrm{H}\right), 7.65-7.69$ (m, $2 \mathrm{H},[\mathrm{h}+\mathrm{k}]-\mathrm{H}), 7.83-7.79(\mathrm{~m}, 7 \mathrm{H},[\mathrm{c}+\mathrm{m}+\mathrm{n}+\mathrm{f} / \mathrm{g}]-\mathrm{H}), 7.88(\mathrm{~s}, 4 \mathrm{H},[5+6]-\mathrm{H}), 7.97\left(\mathrm{~d},{ }^{3} \mathrm{~J}=8.6 \mathrm{~Hz}\right.$, $2 \mathrm{H}, \mathrm{g} / \mathrm{f}-\mathrm{H}), 8.27$ (d, $\left.{ }^{3} J=8.2 \mathrm{~Hz}, 2 \mathrm{H}, 7 / 4-\mathrm{H}\right), 8.32\left(\mathrm{~d},{ }^{3} J=8.2 \mathrm{~Hz}, 2 \mathrm{H}, 4 / 7-\mathrm{H}\right), 8.66-8.63(\mathrm{~m}$, 4H, [a+d]-H), 8.79 (s, 2H, e-H) ppm. ${ }^{13} \mathbf{C}$ NMR (CD2Cl2, 100 MHz): $\delta=17.6,18.6,20.4$, 21.2, 89.6, 90.0, 90.7, 92.7, 93.6, 95.6, 118.6, 121.3, 122.6, 124.2, 124.3 (2C), 124.7, 124.9, $125.4,125.8,126.2,126.5$ (2C), 126.6, 127.5, 127.6, 127.7, 128.6, 128.7, 128.9, 132.1, 132.2, 132.3, 132.8, 133.8, 134.0, 136.0, 136.3, 136.7, 137.0, 137.7, 138.5, 138.7, 142.1, 146.6 (2C), $149.1,149.5,156.2,156.4,160.5,161.3$ ppm. Elemental analysis: Anal. Calcd for $\mathrm{C}_{103} \mathrm{H}_{79} \mathrm{~N}_{7}: \mathrm{C}, 87.44 ; \mathrm{H}, 5.63 ; \mathrm{N}, 6.93$. Found: C, 87.52; H, 5.27; N, 6.75. ESI-MS: $m / z(\%)=$ $1415.0(10)[(\mathbf{1}+\mathrm{H})]^{+}, 708.8(100)[(\mathbf{1}+2 \mathrm{H})]^{2+}$.

\section{Spectroscopic data for ligand 1'.}

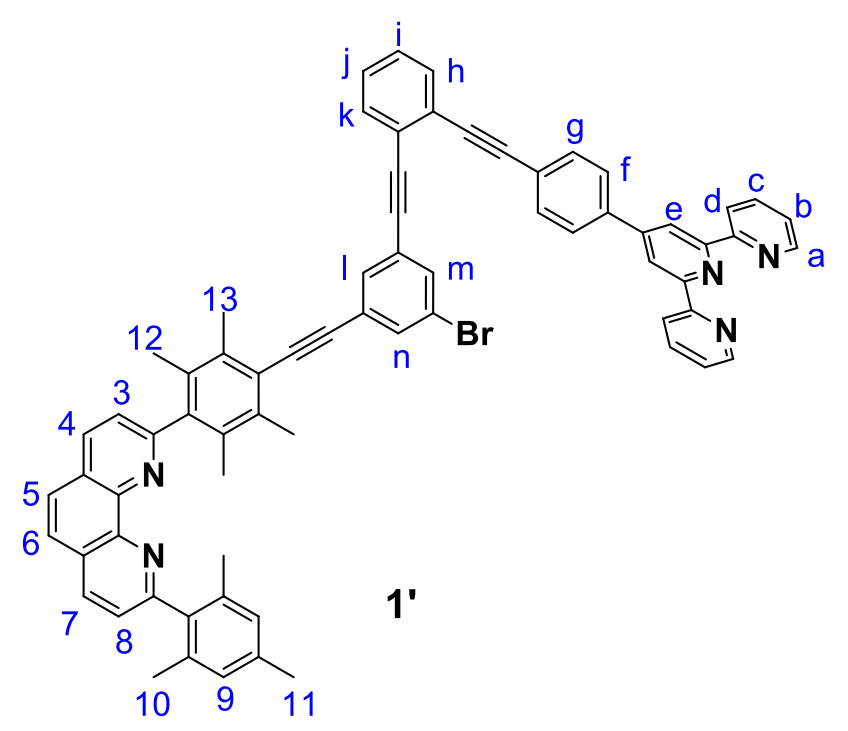

Mp. $>250{ }^{\circ} \mathrm{C} . \mathbf{I R}(\mathbf{K B r}): \tilde{v}=623,787,819,957,1231,1381,1454,1491,1507,1525,2016$, 2154, 2217, 2851, 2916, 2996 cm $^{-1} .{ }^{1} \mathbf{H}-\mathbf{N M R}\left(400 \mathrm{MHz}, \mathbf{C D}_{2} \mathbf{C l}_{2}\right): \delta=1.79(\mathrm{~s}, 6 \mathrm{H}, 13-\mathrm{H})$, $2.01(\mathrm{~s}, 6 \mathrm{H}, 10-\mathrm{H}), 2.30(\mathrm{~s}, 3 \mathrm{H}, 11-\mathrm{H}), 2.44$ (s, 6H, 12-H), $6.92(\mathrm{~s}, 2 \mathrm{H}, 9-\mathrm{H}), 7.33$ (ddd, ${ }^{3} J=$ $\left.7.6 \mathrm{~Hz},{ }^{3} J=4.8 \mathrm{~Hz},{ }^{4} J=1.2 \mathrm{~Hz}, 2 \mathrm{H}, \mathrm{b}-\mathrm{H}\right), 7.39-7.42(\mathrm{~m}, 2 \mathrm{H},[\mathrm{i}+\mathrm{j}]-\mathrm{H}), 7.43\left(\mathrm{~d},{ }^{3} J=8.2 \mathrm{~Hz}\right.$, $1 \mathrm{H}, 8 / 3-\mathrm{H}), 7.55\left(\mathrm{~d},{ }^{3} \mathrm{~J}=8.2 \mathrm{~Hz}, 1 \mathrm{H}, 3 / 8-\mathrm{H}\right), 7.61-7.65(\mathrm{~m}, 2 \mathrm{H},[\mathrm{h}+\mathrm{k}]-\mathrm{H}), 7.71-7.74(\mathrm{~m}, 3 \mathrm{H}$, $[1+\mathrm{m}+\mathrm{n}]-\mathrm{H}), 7.78\left(\mathrm{~d},{ }^{3} \mathrm{~J}=8.6 \mathrm{~Hz}, 2 \mathrm{H}, \mathrm{f} / \mathrm{g}-\mathrm{H}\right), 7.86\left(\mathrm{td},{ }^{3} \mathrm{~J}=7.6 \mathrm{~Hz},{ }^{4} \mathrm{~J}=1.2 \mathrm{~Hz}, 2 \mathrm{H}, \mathrm{c}-\mathrm{H}\right)$, 7.90 (s, 2H, [5+6]-H), $7.96\left(\mathrm{~d},{ }^{3} J=8.6 \mathrm{~Hz}, 2 \mathrm{H}, \mathrm{g} / \mathrm{f}-\mathrm{H}\right), 8.29\left(\mathrm{~d},{ }^{3} J=8.2 \mathrm{~Hz}, 1 \mathrm{H}, 7 / 4-\mathrm{H}\right), 8.33$ $\left(\mathrm{d},{ }^{3} \mathrm{~J}=8.2 \mathrm{~Hz}, 1 \mathrm{H}, 4 / 7-\mathrm{H}\right), 8.65-8.69(\mathrm{~m}, 4 \mathrm{H},[\mathrm{a}+\mathrm{d}]-\mathrm{H}), 8.77$ (s, 2H, e-H) ppm. ${ }^{13} \mathbf{C} \mathbf{~ N M R}$ $\left(\mathbf{C D}_{2} \mathrm{Cl}_{2}, 100 \mathrm{MHz}\right): \delta=17.3,18.2,20.1,20.9,89.6,90.0,91.2,91.5,93.6,94.5,118.5$, $121.1,122.1,123.9,124.0,124.4,124.7,125.2$, 125.3, 126.0, 126.2, 126.3, 126.4, 127.2, 
127.3, 127.4, 127.5 (2C), 128.3, 128.5, 128.8, 131.9, 131.9, 132.0, 132.3, 132.8, 133.6, 133.9, $135.8,136.1,136.5,136.9,137.5,138.1,138.7$, 141.9, 146.2, 146.3, 149.1, 149.2, 156.0, 156.1, 160.3, 160.1 ppm. Elemental analysis: Anal. Calcd for $\mathrm{C}_{70} \mathrm{H}_{50} \mathrm{BrN}_{5} \cdot \mathrm{H}_{2} \mathrm{O}: \mathrm{C}, 79.38 ; \mathrm{H}$, 4.95; N, 6.61. Found: C, 79.49; H, 4.89; N, 6.77. ESI-MS: $m / z(\%)=1042.7(100)\left[\left(\mathbf{1}^{\prime}+\mathrm{H}\right)\right]^{+}$.

\section{Synthesis and characterization of complexes}

Lithium rotor complex: $\left[\mathrm{Li}_{2}(1)\right]^{2+}$

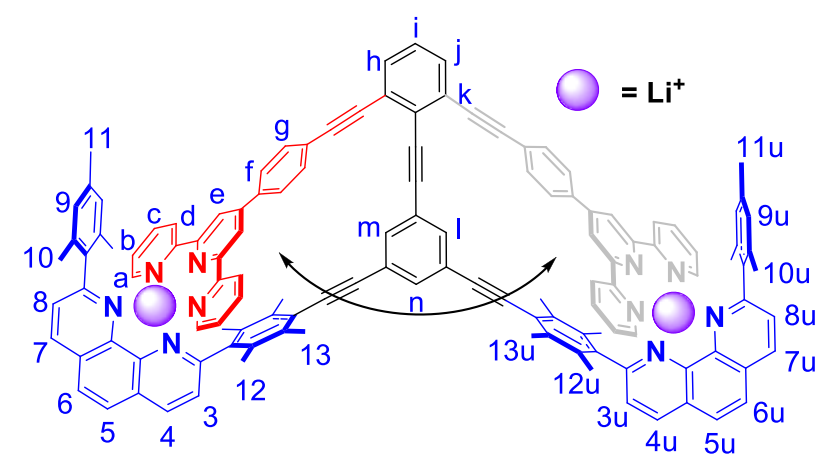

HETTAP rotor $1(0.57 \mathrm{mg}, 0.40 \mu \mathrm{mol})$ and $\mathrm{LiPF}_{6}(0.12 \mathrm{mg}, 0.80 \mu \mathrm{mol})$ were placed in an NMR tube and dissolved in $\mathrm{CD}_{2} \mathrm{Cl}_{2}: \mathrm{CD}_{3} \mathrm{CN}(5: 1$, v/v, $400 \mu \mathrm{L})$ and the NMR spectrum was recorded without purification. ${ }^{1} \mathbf{H}-N M R\left(500 ~ M H z, \mathbf{C D}_{2} \mathbf{C l}_{2}: \mathbf{C D}_{3} \mathbf{C N}(\mathbf{5 : 1})\right): \delta=1.03$ (s, 6H, 13-H), 1.61 (s \{merged with $\left.\left.\mathrm{H}_{2} \mathrm{O}\right\}, 6 \mathrm{H}, 10-\mathrm{H}\right), 1.71$ (s, 6H, 12-H), 1.88 (s, 6H, 13u/12u-H), 1.90 (s, 6H, 12u/13u-H), 1.98 (s, 3H, 11-H), 2.34 (s, 3H, 11u-H), 2.55 (s, 6H, 10u-H), 6.48 (s, 2H, 9-H), 6.98 (s, 2H, 9u-H), 7.09 (ddd, $\left.{ }^{3} J=7.2,{ }^{3} \mathrm{~J}=4.8 \mathrm{~Hz},{ }^{4} \mathrm{~J}=1.2 \mathrm{~Hz}, 2 \mathrm{H}, \mathrm{b}-\mathrm{H}\right), 7.32$ (t, $\left.{ }^{4} J=1.2 \mathrm{~Hz}, 1 \mathrm{H}, \mathrm{m}-\mathrm{H}\right), 7.41\left(\mathrm{~d},{ }^{3} J=8.4 \mathrm{~Hz}, 1 \mathrm{H}, 8 / 3-\mathrm{H}\right), 7.44-7.42(\mathrm{~m}, 2 \mathrm{H}, \mathrm{i}+\mathrm{j}-\mathrm{H}), 7.54\left(\mathrm{dd},{ }^{3} J\right.$ $\left.=4.8 \mathrm{~Hz},{ }^{4} J=1.2 \mathrm{~Hz}, 2 \mathrm{H}, \mathrm{a}-\mathrm{H}\right), 7.60-7.58(\mathrm{~m}, 2 \mathrm{H}, \mathrm{h}+\mathrm{k}-\mathrm{H}), 7.65\left(\mathrm{t},{ }^{4} J=1.2 \mathrm{~Hz}, 1 \mathrm{H}, 1 / \mathrm{n}-\mathrm{H}\right)$, 7.67 (br, 2H, 3u+8u -H), $7.75\left(\mathrm{t},{ }^{4} J=1.2 \mathrm{~Hz}, 1 \mathrm{H}, \mathrm{n} / \mathrm{l}-\mathrm{H}\right), 7.82\left(\mathrm{~d},{ }^{3} J=8.4 \mathrm{~Hz}, 1 \mathrm{H}, 3 / 8-\mathrm{H}\right)$, $7.86\left(\mathrm{td},{ }^{3} J=7.2 \mathrm{~Hz},{ }^{4} J=1.2 \mathrm{~Hz}, 2 \mathrm{H}, \mathrm{c}-\mathrm{H}\right), 7.90\left(\mathrm{~d},{ }^{3} J=8.4 \mathrm{~Hz}, 2 \mathrm{H}, \mathrm{g} / \mathrm{f}-\mathrm{H}\right), 8.02\left(\mathrm{~d},{ }^{3} J=8.4\right.$ $\mathrm{Hz}, 2 \mathrm{H}, \mathrm{f} / \mathrm{g}-\mathrm{H}), 8.03$ (s, 2H, [5u+6u], merged with f/g), $8.05\left(\mathrm{dd}, 2 \mathrm{H},{ }^{3} \mathrm{~J}=7.2 \mathrm{~Hz},{ }^{4} \mathrm{~J}=1.2 \mathrm{~Hz}\right.$, d-H), 8.14 (d, $\left.{ }^{3} J=8.2 \mathrm{~Hz}, 1 \mathrm{H}, 6 / 5-\mathrm{H}\right), 8.15$ (s, 2H, e-H), 8.22 (d, $\left.{ }^{3} J=8.2 \mathrm{~Hz}, 1 \mathrm{H}, 5 / 6-\mathrm{H}\right)$, $8.50\left(\mathrm{~d},{ }^{3} J=8.4 \mathrm{~Hz}, 1 \mathrm{H}, 7 / 4-\mathrm{H}\right), 8.53(\mathrm{br}, 2 \mathrm{H}, 4 \mathrm{u}+7 \mathrm{u}-\mathrm{H}), 8.70\left(\mathrm{~d},{ }^{3} J=8.4 \mathrm{~Hz}, 1 \mathrm{H}, 4 / 7-\mathrm{H}\right)$ ppm. Elemental analysis: Anal. Calcd for $\mathrm{C}_{103} \mathrm{H}_{79} \mathrm{~F}_{12} \mathrm{Li}_{2} \mathrm{~N}_{7} \mathrm{P}_{2} \cdot 2 \mathrm{H}_{2} \mathrm{O}: \mathrm{C}, 70.51 ; \mathrm{H}, 4.77 ; \mathrm{N}$, 5.59. Found: C, 70.56; H, 4.68; N, 5.42. ESI-MS: $m / z(\%)=711.8(100)[\operatorname{Li}(\mathrm{H})(\mathbf{1})]^{2+}$. 


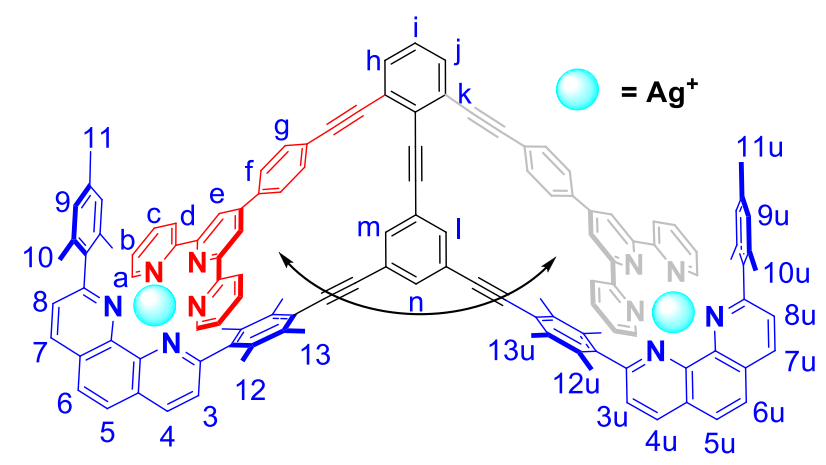

Ligand $1(0.80 \mathrm{mg}, 0.57 \mu \mathrm{mol})$ and $\mathrm{AgBF}_{4}(0.22 \mathrm{mg}, 1.1 \mu \mathrm{mol})$ were mixed in $\mathrm{CD}_{2} \mathrm{Cl}_{2}$ : $\mathrm{CD}_{3} \mathrm{CN}(5: 1, \mathrm{v} / \mathrm{v}, 450 \mu \mathrm{L})$. The NMR spectrum was immediately recorded. ${ }^{1} \mathrm{H}-\mathbf{N M R}(\mathbf{5 0 0}$ MHz, CD $\mathbf{C l}_{2}: \mathrm{CD}_{3} \mathrm{CN}$ (5:1)): $\delta=1.43$ (s, 6H, 13-H), 1.79 (s, 12H, 10+12-H), 1.83 (s, 12H, $12 \mathrm{u}+13 \mathrm{u}-\mathrm{H}), 2.11(\mathrm{~s}, 3 \mathrm{H}, 11-\mathrm{H}), 2.40(\mathrm{~s}, 3 \mathrm{H}, 11 \mathrm{u}-\mathrm{H}), 2.59$ (s, 6H, 10u-H), 6.52 (s, 2H, 9-H), 7.01 (s, 2H, 9u-H), 7.19 (dd, $\left.{ }^{3} J=8.0 \mathrm{~Hz},{ }^{3} J=4.8 \mathrm{~Hz}, 2 \mathrm{H}, \mathrm{b}-\mathrm{H}\right), 7.42-7.46(\mathrm{~m}, 2 \mathrm{H},[\mathrm{i}+\mathrm{j}]-\mathrm{H})$, 7.59 (br, 1H, m-H), 7.60 (br, 1H, 1/n-H) 7.60-7.64 (m, 2H, [h+k]-H), $7.76\left(\mathrm{dd},{ }^{3} J=4.8 \mathrm{~Hz},{ }^{3} J\right.$ $=1.2 \mathrm{~Hz}, 2 \mathrm{H}, \mathrm{a}-\mathrm{H}), 7.84\left(\mathrm{td},{ }^{3} \mathrm{~J}=8.0 \mathrm{~Hz},{ }^{4} \mathrm{~J}=1.2 \mathrm{~Hz}, 2 \mathrm{H}, \mathrm{c}-\mathrm{H}\right), 7.87$ (d, ${ }^{3} \mathrm{~J}=8.4 \mathrm{~Hz}, 2 \mathrm{H}, \mathrm{g} / \mathrm{f}-$ H), 7.89 (br, 5H, [n/l+3+8+3u+8u]-H, merged with g/f), 8.03 (d, $\left.{ }^{3} J=8.4 \mathrm{~Hz}, 2 \mathrm{H}, \mathrm{f} / \mathrm{g}-\mathrm{H}\right), 8.09$ $\left(\mathrm{d},{ }^{3} \mathrm{~J}=8.0 \mathrm{~Hz}, 2 \mathrm{H}, \mathrm{d}-\mathrm{H}\right), 8.14$ (br, 4H, [5+6+5u+6u]-H), 8.26 (s, 2H, e-H), 8.59 (br, 2H, $[7 / 4+7 / 4 \mathrm{u}]-\mathrm{H}), 8.69$ (br, 2H, [4/7+4/7u]-H) ppm. Elemental analysis: Anal. Calcd for $\mathrm{C}_{103} \mathrm{H}_{79} \mathrm{Ag}_{2} \mathrm{~B}_{2} \mathrm{~F}_{8} \mathrm{~N}_{7} \cdot \mathrm{CH}_{2} \mathrm{Cl}_{2}$ : C, 66.12; H, 4.32; N, 5.19. Found: C, 65.85; H, 4.64; N, 5.45. ESI-MS: $m / z(\%)=815.4(100)\left[\operatorname{Ag}_{2}(\mathbf{1})\right]^{+2}$. 


\section{Synthesis of rotor $\left[\mathrm{H}_{2}(1)\right]^{2+}$}

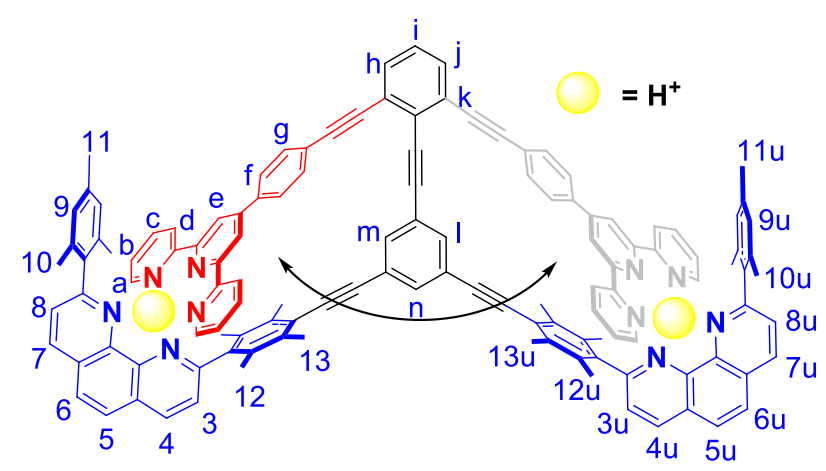

Ligand $1(0.750 \mathrm{mg}, 0.530 \mu \mathrm{mol})$ was placed in an NMR tube and dissolved in deuterated $\mathrm{CD}_{2} \mathrm{Cl}_{2}$. To that solution, 2.0 equivalent of trifluoroacetic acid $(0.121 \mathrm{mg}, 1.06 \mu \mathrm{mol})$ was added. The NMR spectrum was recorded immediately. ${ }^{1} \mathrm{H}-\mathrm{NMR}\left(\mathbf{5 0 0} \mathbf{M H z}, \mathbf{C D}_{2} \mathbf{C l}_{2}\right): \delta=$ $1.61(\mathrm{~s}, 12 \mathrm{H},[13+13 \mathrm{u}]-\mathrm{H}) 1.95(\mathrm{~s}, 12 \mathrm{H},[10+10 \mathrm{u}]-\mathrm{H}), 2.23(\mathrm{~s}, 18 \mathrm{H},[11+11 \mathrm{u}+12+12 \mathrm{u}]-\mathrm{H})$, $6.16\left(\mathrm{br}\right.$, appeared at $\left.-75^{\circ} \mathrm{C}, 1 \mathrm{H}, \mathrm{m} / \mathrm{l}-\mathrm{H}\right), 6.78(\mathrm{~s}, 4 \mathrm{H},[9+9 \mathrm{u}]-\mathrm{H}), 6.97$ (br, appeared at -75 $\left.{ }^{\circ} \mathrm{C}, 1 \mathrm{H}, 1 / \mathrm{m}-\mathrm{H}\right), 7.21\left(\mathrm{dd},{ }^{3} \mathrm{~J}=8.0 \mathrm{~Hz},{ }^{3} J=4.8 \mathrm{~Hz}, 2 \mathrm{H}, \mathrm{b}-\mathrm{H}\right), 7.41-7.45(\mathrm{~m}, 2 \mathrm{H},[\mathrm{i}+\mathrm{j}]-\mathrm{H})$, $7.50\left(\mathrm{~d},{ }^{3} \mathrm{~J}=8.2 \mathrm{~Hz}, 2 \mathrm{H},[8 / 3+8 / 3 \mathrm{u}]-\mathrm{H}\right), 7.60-7.67(\mathrm{~m}, 2 \mathrm{H},[\mathrm{h}+\mathrm{k}]-\mathrm{H}), 7.69\left(\mathrm{~d},{ }^{3} J=8.2 \mathrm{~Hz}, 2 \mathrm{H}\right.$, [3/8+3/8u]-H), 7.70 (br, merged with 3/8-H, 2H, c-H), $7.72\left(\mathrm{t},{ }^{4} \mathrm{~J}=1.6 \mathrm{~Hz}, 1 \mathrm{H}, \mathrm{n}-\mathrm{H}\right), 7.90$ (d, $\left.{ }^{3} J=8.4 \mathrm{~Hz}, 2 \mathrm{H}, \mathrm{g}-\mathrm{H}\right), 7.91$ (br, merged with g-H, $\left.2 \mathrm{H}, \mathrm{d}-\mathrm{H}\right), 8.02\left(\mathrm{~d},{ }^{3} J=8.4 \mathrm{~Hz}, 2 \mathrm{H}\right.$, [5/6+5/6u]-H), $8.08\left(\mathrm{~d},{ }^{3} J=8.4 \mathrm{~Hz}, 2 \mathrm{H}, \mathrm{f}-\mathrm{H}\right), 8.09$ (d, $\left.{ }^{3} J=8.4 \mathrm{~Hz}, 2 \mathrm{H},[6 / 5+6 / 5 \mathrm{u}]-\mathrm{H}\right), 8.42$ (d, $\left.{ }^{3} J=8.2 \mathrm{~Hz}, 2 \mathrm{H},[7 / 4+7 \mathrm{u} / 4 \mathrm{u}]-\mathrm{H}\right), 8.50(\mathrm{br}, 2 \mathrm{H}, \mathrm{e}-\mathrm{H}), 8.56\left(\mathrm{~d},{ }^{3} J=8.2 \mathrm{~Hz}, 2 \mathrm{H},[4 / 7+4 \mathrm{u} / 7 \mathrm{u}]-\mathrm{H}\right)$, $8.59\left(\mathrm{dd},{ }^{3} \mathrm{~J}=4.8 \mathrm{~Hz},{ }^{4} \mathrm{~J}=1.2 \mathrm{~Hz}, 2 \mathrm{H}, \mathrm{a}-\mathrm{H}\right) \mathrm{ppm}$. Elemental analysis: Anal. Calcd for $\mathrm{C}_{107} \mathrm{H}_{81} \mathrm{~F}_{6} \mathrm{~N}_{7} \mathrm{O}_{4} \cdot \mathrm{H}_{2} \mathrm{O}: \mathrm{C}, 77.38 ; \mathrm{H}, 5.04 ; \mathrm{N}, 5.90$. Found: C, 77.14; H, 4.79; N, 5.84. ESI-MS: $m / z(\%)=1415.0(10)[(\mathbf{1}+\mathrm{H})]^{+}, 708.8(100)[(\mathbf{1}+2 \mathrm{H})]^{2+}$. 


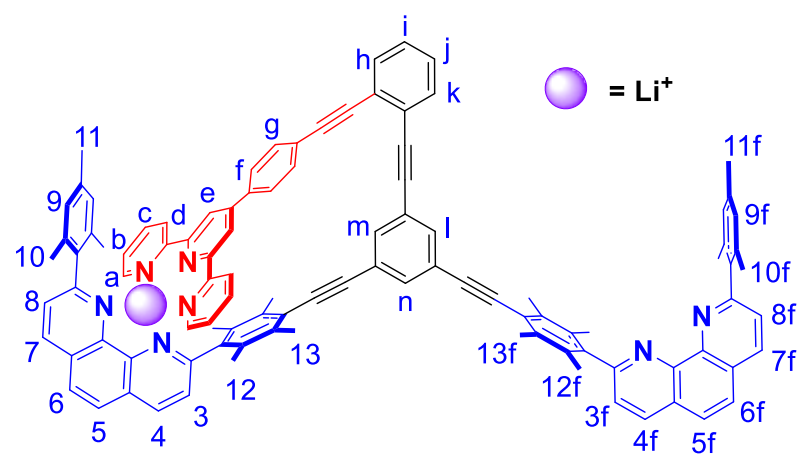

HETTAP rotor $1(0.57 \mathrm{mg}, 0.40 \mu \mathrm{mol})$ and $\mathrm{LiPF}_{6}(0.060 \mathrm{mg}, 0.40 \mu \mathrm{mol})$ were placed in an NMR tube and dissolved in $\mathrm{CD}_{2} \mathrm{Cl}_{2}: \mathrm{CD}_{3} \mathrm{CN}(5: 1, \mathrm{v} / \mathrm{v}, 400 \mu \mathrm{L})$ and the NMR spectrum was recorded without purification. ${ }^{1} \mathrm{H}-\mathrm{NMR}\left(\mathbf{5 0 0} \mathrm{MHz}, \mathbf{C D}_{2} \mathrm{Cl}_{2}: \mathbf{C D}_{3} \mathrm{CN}=\mathbf{5 : 1}\right): \delta=1.04(\mathrm{~s}, 6 \mathrm{H}$, 13-H), 1.72 (s, 6H, 10-H), 1.88 (s, 6H, 12-H), 1.95 (s, merged with $\left.\mathrm{CD}_{3} \mathrm{CN}, 6 \mathrm{H}, 10 \mathrm{f}-\mathrm{H}\right), 2.03$ (s, 6H, 13f-H), 2.11 (s, 3H, 11-H), 2.33 (s, 3H, 11f-H), 2.54 (s, 6H, 12f-H), 6.48 (s, 2H, 9-H), 6.95 (s, 2H, 9f-H), 7.09 (ddd, $\left.{ }^{3} J=7.2 \mathrm{~Hz},{ }^{3} J=4.8 \mathrm{~Hz},{ }^{4} J=1.2 \mathrm{~Hz}, 2 \mathrm{H}, \mathrm{b}-\mathrm{H}\right), 7.32\left(\mathrm{t},{ }^{4} J=1.6\right.$ $\mathrm{Hz}, 1 \mathrm{H}, \mathrm{m}-\mathrm{H}), 7.42\left(\mathrm{~d},{ }^{3} \mathrm{~J}=8.4 \mathrm{~Hz}, 1 \mathrm{H}, 8 / 3-\mathrm{H}\right), 7.43-7.45(\mathrm{~m}, 2 \mathrm{H}, \mathrm{i}+\mathrm{j}-\mathrm{H}), 7.53-7.55(\mathrm{~m}, 3 \mathrm{H}$, $\mathrm{a}-\mathrm{H}+8 \mathrm{f} / 3 \mathrm{f}-\mathrm{H}), 7.56\left(\mathrm{~d},{ }^{3} \mathrm{~J}=8.2 \mathrm{~Hz}, 1 \mathrm{H}, 3 \mathrm{f} / 8 \mathrm{f}-\mathrm{H}\right), 7.58-7.61(\mathrm{~m}, 1 \mathrm{H}, \mathrm{k} / \mathrm{h}-\mathrm{H}), 7.65-7.67$ (m, $2 \mathrm{H}, \mathrm{l} / \mathrm{n}-\mathrm{H}+\mathrm{h} / \mathrm{k}-\mathrm{H}), 7.77\left(\mathrm{t},{ }^{4} \mathrm{~J}=1.6 \mathrm{~Hz}, 1 \mathrm{H}, \mathrm{n} / \mathrm{l}-\mathrm{H}\right), 7.82\left(\mathrm{~d},{ }^{3} J=8.4 \mathrm{~Hz}, 1 \mathrm{H}, 3 / 8-\mathrm{H}\right), 7.86(\mathrm{td}$, $\left.{ }^{3} J=7.2 \mathrm{~Hz},{ }^{4} J=1.2 \mathrm{~Hz}, 2 \mathrm{H}, \mathrm{c}-\mathrm{H}\right), 7.92\left(\mathrm{~d},{ }^{3} J=8.4 \mathrm{~Hz}, 2 \mathrm{H}, \mathrm{g} / \mathrm{f}-\mathrm{H}\right), 7.93$ (s, 2H, 5f+6f-H), $8.02\left(\mathrm{~d},{ }^{3} \mathrm{~J}=8.4 \mathrm{~Hz}, 2 \mathrm{H}, \mathrm{f} / \mathrm{g}-\mathrm{H}\right), 8.04\left(\mathrm{dd},{ }^{3} \mathrm{~J}=7.2 \mathrm{~Hz},{ }^{4} \mathrm{~J}=1.2 \mathrm{~Hz}, 2 \mathrm{H}, \mathrm{d}-\mathrm{H}\right), 8.15$ (s, 2H, e$\mathrm{H}), 8.16\left(\mathrm{~d},{ }^{3} \mathrm{~J}=8.8 \mathrm{~Hz}, 1 \mathrm{H}, 5 / 6-\mathrm{H}\right), 8.23\left(\mathrm{~d},{ }^{3} J=8.8 \mathrm{~Hz}, 1 \mathrm{H}, 6 / 5-\mathrm{H}\right), 8.35\left(\mathrm{~d},{ }^{3} J=8.4 \mathrm{~Hz}\right.$, $1 \mathrm{H}, 7 \mathrm{f} / 4 \mathrm{f}-\mathrm{H}), 8.37$ (d, $\left.{ }^{3} J=8.4 \mathrm{~Hz}, 1 \mathrm{H}, 4 \mathrm{f} / 7 \mathrm{f}-\mathrm{H}\right), 8.50\left(\mathrm{~d},{ }^{3} J=8.4 \mathrm{~Hz}, 1 \mathrm{H}, 7 / 4-\mathrm{H}\right), 8.72\left(\mathrm{~d},{ }^{3} J=\right.$ $8.4 \mathrm{~Hz}, 1 \mathrm{H}, 4 / 7-\mathrm{H})$ ppm. ESI-MS: $m / z(\%)=711.8(100)[\mathrm{Li}(\mathrm{H})(\mathbf{1})]^{2+}$. 


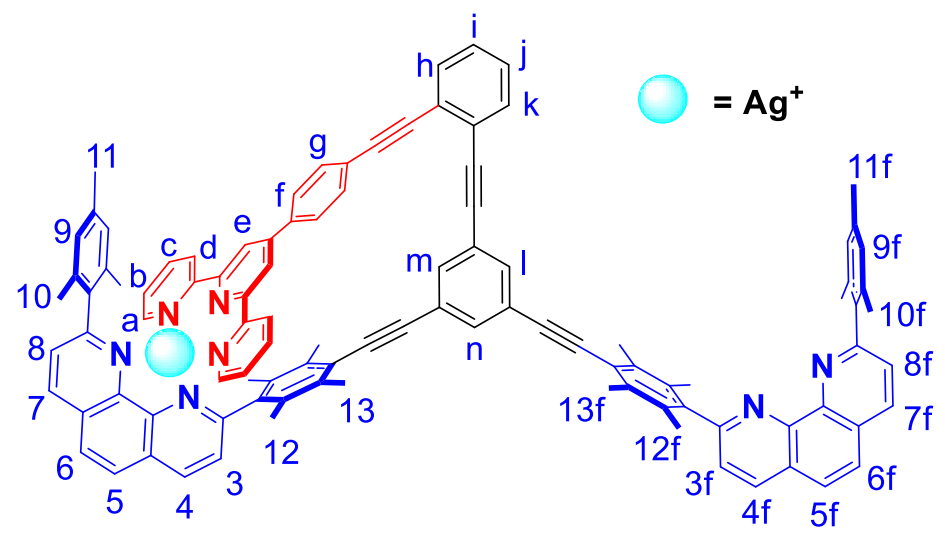

Ligand $1(0.80 \mathrm{mg}, 0.57 \mu \mathrm{mol})$ and $\mathrm{AgBF}_{4}(0.11 \mathrm{mg}, 0.57 \mu \mathrm{mol})$ were mixed in $\mathrm{CD}_{2} \mathrm{Cl}_{2}$ : $\mathrm{CD}_{3} \mathrm{CN}(5: 1, \mathrm{v} / \mathrm{v}, 450 \mu \mathrm{L})$. The NMR spectrum was immediately recorded. ${ }^{1} \mathbf{H}-\mathbf{N M R}$ (500 MHz, CD2 $\left.\mathbf{C l}_{2}: \mathrm{CD}_{3} \mathrm{CN}=\mathbf{5 : 1}\right): \delta=1.38$ (s, 6H, 13-H), 1.80 (s, 6H, 10-H), 1.86 (s, 6H, 12H), 1.92 (s, 3H, 11-H), 1.94 (s, 6H, 13f-H), 2.03 (s, 6H, 10f-H), 2.33 (s, 3H, 11f-H), 2.53 (s, $6 \mathrm{H}, 12 \mathrm{f}-\mathrm{H}), 6.56$ (s, 2H, 9-H), 6.95 (s, 2H, 9f-H), 7.17 (t, $\left.{ }^{4} J=1.6 \mathrm{~Hz} 1 \mathrm{H}, \mathrm{m}-\mathrm{H}\right), 7.22\left(\mathrm{dd},{ }^{3} J\right.$ $\left.=8.0 \mathrm{~Hz},{ }^{3} \mathrm{~J}=4.8 \mathrm{~Hz}, 2 \mathrm{H}, \mathrm{b}-\mathrm{H}\right), 7.42-7.45(\mathrm{~m}, 2 \mathrm{H},[\mathrm{i}+\mathrm{j}]-\mathrm{H}), 7.53-7.56(\mathrm{~m}, 2 \mathrm{H},[3 \mathrm{f}+8 \mathrm{f}]-\mathrm{H})$, 7.57-7.60 (m, 2H, [8/3 + k/h]-H), $7.62\left(\mathrm{t},{ }^{4} J=1.6 \mathrm{~Hz} 1 \mathrm{H}, \mathrm{n} / \mathrm{l}-\mathrm{H}\right), 7.64-7.66(\mathrm{~m}, 1 \mathrm{H},[\mathrm{h} / \mathrm{k}]-\mathrm{H})$, $7.76\left(\mathrm{t},{ }^{4} J=1.6 \mathrm{~Hz} 1 \mathrm{H}, 1 / \mathrm{n}-\mathrm{H}\right), 7.79\left(\mathrm{dd},{ }^{3} J=4.8 \mathrm{~Hz},{ }^{3} J=1.2 \mathrm{~Hz}, 2 \mathrm{H}, \mathrm{a}-\mathrm{H}\right), 7.83\left(\mathrm{td},{ }^{3} J=8.0\right.$ $\left.\mathrm{Hz},{ }^{4} J=1.2 \mathrm{~Hz}, 2 \mathrm{H}, \mathrm{c}-\mathrm{H}\right), 7.88$ (d, $\left.{ }^{3} J=8.4 \mathrm{~Hz}, 2 \mathrm{H}, \mathrm{g} / \mathrm{f}-\mathrm{H}\right), 7.92\left(\mathrm{~d},{ }^{3} J=8.2 \mathrm{~Hz} 1 \mathrm{H},[3 / 8]-\mathrm{H}\right)$, $7.92(\mathrm{~s}, 2 \mathrm{H},[5 \mathrm{f}+6 \mathrm{f}]-\mathrm{H}), 8.01\left(\mathrm{~d},{ }^{3} J=8.4 \mathrm{~Hz}, 2 \mathrm{H}, \mathrm{f} / \mathrm{g}-\mathrm{H}\right), 8.07\left(\mathrm{~d},{ }^{3} J=8.0 \mathrm{~Hz}, 2 \mathrm{H}, \mathrm{d}-\mathrm{H}\right), 8.16$ $\left(\mathrm{d},{ }^{3} J=9.0 \mathrm{~Hz}, 1 \mathrm{H},[5 / 6]-\mathrm{H}\right), 8.21\left(\mathrm{~d},{ }^{3} J=9.0 \mathrm{~Hz}, 1 \mathrm{H},[6 / 5]-\mathrm{H}\right), 8.25(\mathrm{~s}, 2 \mathrm{H}, \mathrm{e}-\mathrm{H}), 8.35$ (d, ${ }^{3} J$ $=8.2 \mathrm{~Hz}, 1 \mathrm{H},[7 \mathrm{f} / 4 \mathrm{f}]-\mathrm{H}), 8.37\left(\mathrm{~d},{ }^{3} J=8.2 \mathrm{~Hz}, 1 \mathrm{H},[4 \mathrm{f} / 7 \mathrm{f}]-\mathrm{H}\right), 8.54\left(\mathrm{~d},{ }^{3} J=8.2 \mathrm{~Hz}, 1 \mathrm{H},[7 / 4]-\right.$ $\mathrm{H}), 8.71\left(\mathrm{~d},{ }^{3} \mathrm{~J}=8.2 \mathrm{~Hz}, 1 \mathrm{H},[4 / 7]-\mathrm{H}\right) \mathrm{ppm}$. 


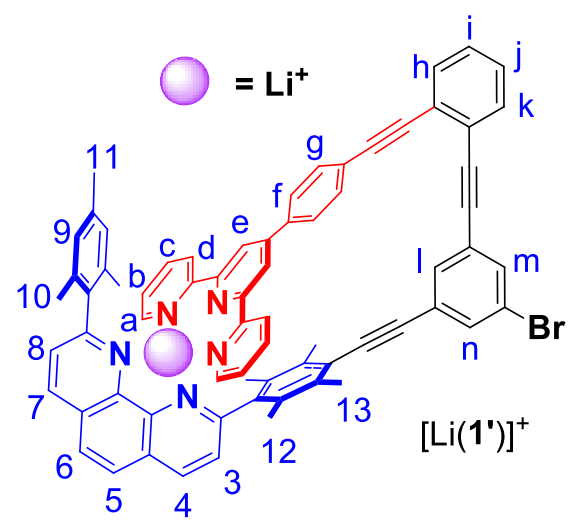

Ligand 1' $(0.450 \mathrm{mg}, 0.43 \mu \mathrm{mol})$ and $\mathrm{LiPF}_{6}(66 \mu \mathrm{g}, 0.43 \mu \mathrm{mol})$ were placed in an NMR tube and dissolved in $\mathrm{CD}_{2} \mathrm{Cl}_{2}: \mathrm{CD}_{3} \mathrm{CN}(5: 1, \mathrm{v} / \mathrm{v}, 400 \mu \mathrm{L})$ and the NMR spectrum was recorded without purification. ${ }^{1} \mathbf{H}-\mathrm{NMR}\left(\mathbf{5 0 0} \mathbf{M H z}, \mathbf{C D}_{\mathbf{2}} \mathbf{C l}_{\mathbf{2}}: \mathbf{C D}_{\mathbf{3}} \mathbf{C N}=\mathbf{5 : 1}\right): \delta=1.03(\mathrm{~s}, 6 \mathrm{H}, 13-\mathrm{H})$, 1.70 (s, 6H, 10-H), 1.84 (s, 6H, 12-H), 1.93 (s, 3H, 11-H), 6.47 (s, 2H, 9-H), 7.09 (ddd, ${ }^{3} J=$ $\left.8.0,{ }^{3} \mathrm{~J}=4.8 \mathrm{~Hz},{ }^{4} \mathrm{~J}=0.8 \mathrm{~Hz}, 2 \mathrm{H}, \mathrm{b}-\mathrm{H}\right), 7.26\left(\mathrm{t},{ }^{4} \mathrm{~J}=1.4 \mathrm{~Hz}, 1 \mathrm{H}, 1-\mathrm{H}\right), 7.41\left(\mathrm{~d},{ }^{3} \mathrm{~J}=8.2 \mathrm{~Hz}\right.$, $1 \mathrm{H}, 8 / 3-\mathrm{H}), 7.42-7.44(\mathrm{~m}, 2 \mathrm{H},[\mathrm{i}+\mathrm{j}]-\mathrm{H}), 7.54\left(\mathrm{ddd},{ }^{3} \mathrm{~J}=4.8 \mathrm{~Hz},{ }^{4} J=1.2 \mathrm{~Hz},{ }^{5} J=0.8 \mathrm{~Hz}, 2 \mathrm{H}\right.$, a-H), 7.57-7.63 (m, 3H, [h+k+m/n]-H), $7.70\left(\mathrm{t},{ }^{4} J=1.4 \mathrm{~Hz}, 1 \mathrm{H}, \mathrm{n} / \mathrm{m}-\mathrm{H}\right), 7.82\left(\mathrm{~d},{ }^{3} J=8.2 \mathrm{~Hz}\right.$, $1 \mathrm{H}, 3 / 8-\mathrm{H}), 7.84\left(\mathrm{td},{ }^{3} J=8.0 \mathrm{~Hz},{ }^{4} J=1.2 \mathrm{~Hz}, 2 \mathrm{H}, \mathrm{c}-\mathrm{H}\right), 7.87\left(\mathrm{~d},{ }^{3} J=8.4 \mathrm{~Hz}, 2 \mathrm{H}, \mathrm{g} / \mathrm{f}-\mathrm{H}\right), 7.99$ $\left(\mathrm{d},{ }^{3} J=8.4 \mathrm{~Hz}, 2 \mathrm{H}, \mathrm{f} / \mathrm{g}-\mathrm{H}\right), 8.03\left(\mathrm{dd},{ }^{3} J=8.0 \mathrm{~Hz},{ }^{4} J=0.8 \mathrm{~Hz}, 2 \mathrm{H}, \mathrm{d}-\mathrm{H}\right), 8.13(\mathrm{~s}, 2 \mathrm{H}, \mathrm{e}-\mathrm{H})$, $8.15\left(\mathrm{~d},{ }^{3} \mathrm{~J}=8.8 \mathrm{~Hz}, 1 \mathrm{H}, 6 / 5-\mathrm{H}\right), 8.23\left(\mathrm{~d},{ }^{3} \mathrm{~J}=8.8 \mathrm{~Hz}, 1 \mathrm{H}, 5 / 6-\mathrm{H}\right), 8.50\left(\mathrm{~d},{ }^{3} \mathrm{~J}=8.2 \mathrm{~Hz}, 1 \mathrm{H}\right.$, 7/4-H), $8.71\left(\mathrm{~d},{ }^{3} J=8.2 \mathrm{~Hz}, 1 \mathrm{H}, 4 / 7-\mathrm{H}\right)$ ppm. ESI-MS: $m / z(\%)=1048.9(100)\left[\operatorname{Li}\left(\mathbf{1}^{\prime}\right)\right]^{+}$.

Silver switch complex: $\left[\operatorname{Ag}\left(\mathbf{1}^{\prime}\right)\right]^{+}$

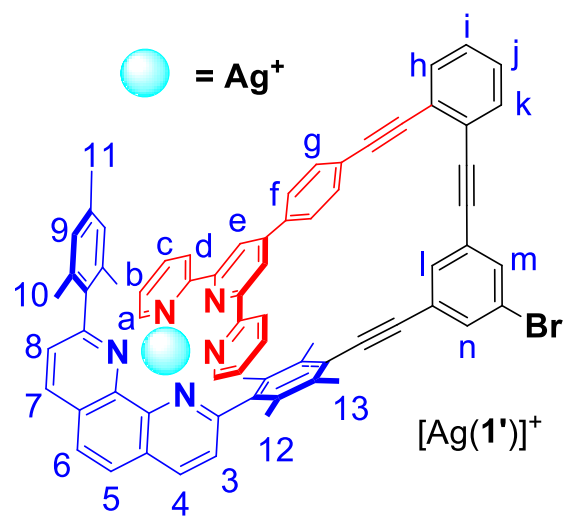

Ligand 1' (0.41 mg, $0.39 \mu \mathrm{mol})$ was dissolved in deuterated DCM. AgBF 4 (70 $\mu \mathrm{g}, 0.39 \mu \mathrm{mol})$ in $\mathrm{CD}_{3} \mathrm{CN}$ was added and the NMR spectrum recorded. ${ }^{1} \mathbf{H}-\mathbf{N M R}(\mathbf{4 0 0} \mathbf{M H z}$, $\left.\mathbf{C D}_{2} \mathrm{Cl}_{2}: \mathbf{C D}_{3} \mathrm{CN}=\mathbf{5 : 1}\right): \delta=1.38(\mathrm{~s}, 6 \mathrm{H}, 13-\mathrm{H}), 1.77(\mathrm{~s}, 6 \mathrm{H}, 12-\mathrm{H}), 1.86(\mathrm{~s}, 6 \mathrm{H}, 10-\mathrm{H}), 1.91$ 
(s, 3H, 11-H), 6.55 (s, 2H, 9-H), $7.11\left(\mathrm{t},{ }^{4} J=1.6 \mathrm{~Hz}, 1 \mathrm{H}, 1-\mathrm{H}\right), 7.22\left(\mathrm{ddd},{ }^{3} J=7.8 \mathrm{~Hz},{ }^{3} J=4.8\right.$ $\left.\mathrm{Hz},{ }^{4} J=1.2 \mathrm{~Hz}, 2 \mathrm{H}, \mathrm{b}-\mathrm{H}\right), 7.42-7.44(\mathrm{~m}, 2 \mathrm{H},[\mathrm{i}+\mathrm{j}]-\mathrm{H}), 7.57-7.63(\mathrm{~m}, 4 \mathrm{H},[\mathrm{h}+\mathrm{k}+\mathrm{n} / \mathrm{m}+8 / 3]-\mathrm{H})$, $7.69\left(\mathrm{t},{ }^{4} J=1.6 \mathrm{~Hz}, 1 \mathrm{H}, \mathrm{m} / \mathrm{n}-\mathrm{H}\right), 7.80\left(\mathrm{dd},{ }^{3} J=4.8 \mathrm{~Hz},{ }^{4} J=1.2 \mathrm{~Hz}, 2 \mathrm{H}, \mathrm{a}-\mathrm{H}\right), 7.84\left(\mathrm{td},{ }^{3} J=\right.$ $\left.7.8 \mathrm{~Hz},{ }^{4} \mathrm{~J}=1.2 \mathrm{~Hz}, 2 \mathrm{H}, \mathrm{c}-\mathrm{H}\right), 7.86\left(\mathrm{~d},{ }^{3} \mathrm{~J}=8.2 \mathrm{~Hz}, 2 \mathrm{H}, \mathrm{g} / \mathrm{f}-\mathrm{H}\right), 7.92\left(\mathrm{~d},{ }^{3} \mathrm{~J}=8.4 \mathrm{~Hz}, 1 \mathrm{H}, 3 / 8-\right.$ H), $7.98\left(\mathrm{~d},{ }^{3} \mathrm{~J}=8.2 \mathrm{~Hz}, 2 \mathrm{H}, \mathrm{f} / \mathrm{g}-\mathrm{H}\right), 8.06\left(\mathrm{dd},{ }^{3} \mathrm{~J}=7.8 \mathrm{~Hz},{ }^{4} \mathrm{~J}=1.2 \mathrm{~Hz}, 2 \mathrm{H}, \mathrm{d}-\mathrm{H}\right), 8.16\left(\mathrm{~d},{ }^{3} J=\right.$ $9.0 \mathrm{~Hz}, 1 \mathrm{H}, 6 / 5-\mathrm{H}), 8.21\left(\mathrm{~d},{ }^{3} \mathrm{~J}=9.0 \mathrm{~Hz}, 1 \mathrm{H}, 5 / 6-\mathrm{H}\right), 8.23$ (s, 2H, e-H), 8.54 (d, ${ }^{3} \mathrm{~J}=8.4 \mathrm{~Hz}$, $1 \mathrm{H}, 7 / 4-\mathrm{H}), 8.71\left(\mathrm{~d},{ }^{3} J=8.4 \mathrm{~Hz}, 1 \mathrm{H}, 4 / 7-\mathrm{H}\right) \mathrm{ppm}$. ESI-MS: $\mathrm{m} / \mathrm{z}(\%)=1148.7(100)$ $\left[\operatorname{Ag}\left(\mathbf{1}^{\prime}\right)\right]^{+}$.

\section{Proton switch complex: $\left[\mathrm{H}\left(1^{\prime}\right)\right]^{+}$}

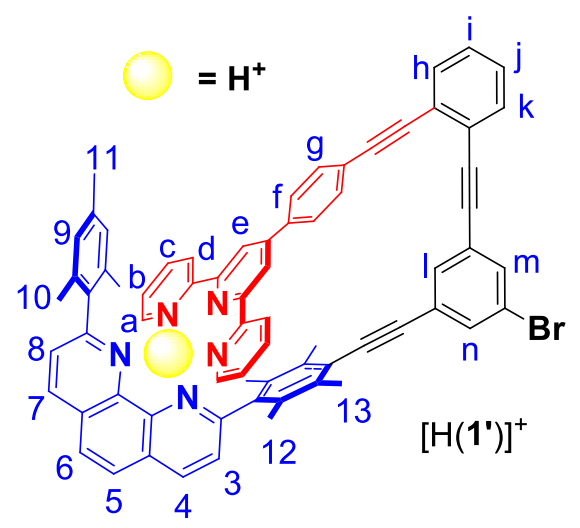

Ligand 1' $(0.52 \mathrm{mg}, 0.50 \mu \mathrm{mol})$ was placed in an NMR tube and dissolved in $\mathrm{CD}_{2} \mathrm{Cl}_{2}$. To that solution, 1.0 equiv of trifluoroacetic acid $(57 \mu \mathrm{g}, 0.50 \mu \mathrm{mol})$ was added. The NMR spectrum was recorded immediately. ${ }^{1} \mathbf{H}-\mathbf{N M R}\left(\mathbf{5 0 0} \mathbf{~ M H z}, \mathbf{C D}_{2} \mathbf{C l}_{2}\right): \delta=1.26(\mathrm{~s}, 6 \mathrm{H}, 13-\mathrm{H}) 1.87$ (s, 6H, 10-H), 1.89 (s, 6H, 12-H), 2.13 (s, 3H, 11-H), 6.60 (s, 2H, 9-H), $7.23\left(\mathrm{dd},{ }^{3} J=8.0 \mathrm{~Hz},{ }^{3} J=\right.$ $4.8 \mathrm{~Hz}, 2 \mathrm{H}, \mathrm{b}-\mathrm{H}), 7.41\left(\mathrm{~d},{ }^{3} \mathrm{~J}=8.2 \mathrm{~Hz}, 1 \mathrm{H}, 8 / 3-\mathrm{H}\right), 7.42-7.44(\mathrm{~m}, 2 \mathrm{H},[\mathrm{i}+\mathrm{j}]-\mathrm{H}), 7.56\left(\mathrm{t},{ }^{4} J=1.4\right.$ $\mathrm{Hz}, 1 \mathrm{H}, 1-\mathrm{H}), 7.59-7.64(\mathrm{~m}, 4 \mathrm{H},[\mathrm{h}+\mathrm{k}+\mathrm{c}]-\mathrm{H}), 7.68\left(\mathrm{t},{ }^{4} J=1.4 \mathrm{~Hz}, 1 \mathrm{H}, \mathrm{n} / \mathrm{m}-\mathrm{H}\right), 7.74\left(\mathrm{t},{ }^{4} J=1.4\right.$ $\mathrm{Hz}, 1 \mathrm{H}, \mathrm{m} / \mathrm{n}-\mathrm{H}), 7.79\left(\mathrm{~d},{ }^{3} J=8.2 \mathrm{~Hz}, 1 \mathrm{H}, 3 / 8-\mathrm{H}\right), 7.87\left(\mathrm{~d},{ }^{3} J=8.4 \mathrm{~Hz}, 2 \mathrm{H}, \mathrm{g}-\mathrm{H}\right), 7.92\left(\mathrm{~d},{ }^{3} J=\right.$ $8.0 \mathrm{~Hz}, 2 \mathrm{H}, \mathrm{d}-\mathrm{H}), 8.06\left(\mathrm{~d},{ }^{3} J=8.4 \mathrm{~Hz}, 2 \mathrm{H}, \mathrm{f}-\mathrm{H}\right), 8.10\left(\mathrm{~d},{ }^{3} J=8.4 \mathrm{~Hz}, 2 \mathrm{H}, 6 / 5-\mathrm{H}\right), 8.22\left(\mathrm{~d},{ }^{3} J\right.$ $=8.4 \mathrm{~Hz}, 1 \mathrm{H}, 5 / 6-\mathrm{H}), 8.43\left(\mathrm{~d},{ }^{3} \mathrm{~J}=8.2 \mathrm{~Hz}, 1 \mathrm{H}, 7 / 4-\mathrm{H}\right), 8.48(\mathrm{br}, 2 \mathrm{H}, \mathrm{e}-\mathrm{H}), 8.60\left(\mathrm{dd},{ }^{3} J=4.8\right.$ $\left.\mathrm{Hz},{ }^{4} J=1.2 \mathrm{~Hz}, 2 \mathrm{H}, \mathrm{a}-\mathrm{H}\right), 8.69\left(\mathrm{~d},{ }^{3} J=8.2 \mathrm{~Hz}, 1 \mathrm{H}, 4 / 7-\mathrm{H}\right) \mathrm{ppm}$. ESI-MS: $m / z(\%)=1042.7$ (100) $\left[\left(\mathbf{1}^{\prime}+\mathrm{H}\right)\right]^{+}$. 


\section{NMR spectra}

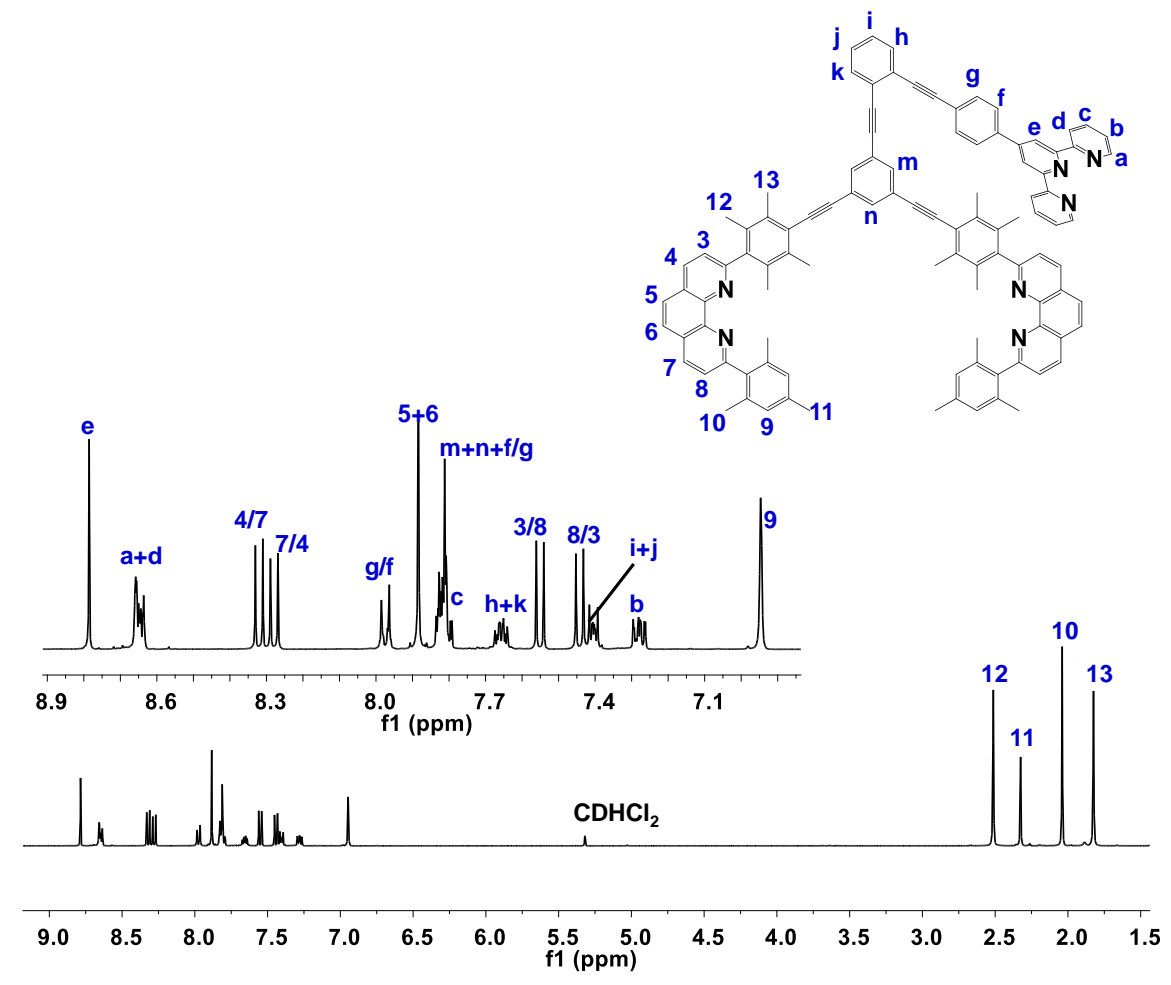

Figure S1. ${ }^{1} \mathrm{H}$ NMR spectrum of 1 in $\mathrm{CD}_{2} \mathrm{Cl}_{2}(400 \mathrm{MHz}, 298 \mathrm{~K})$.

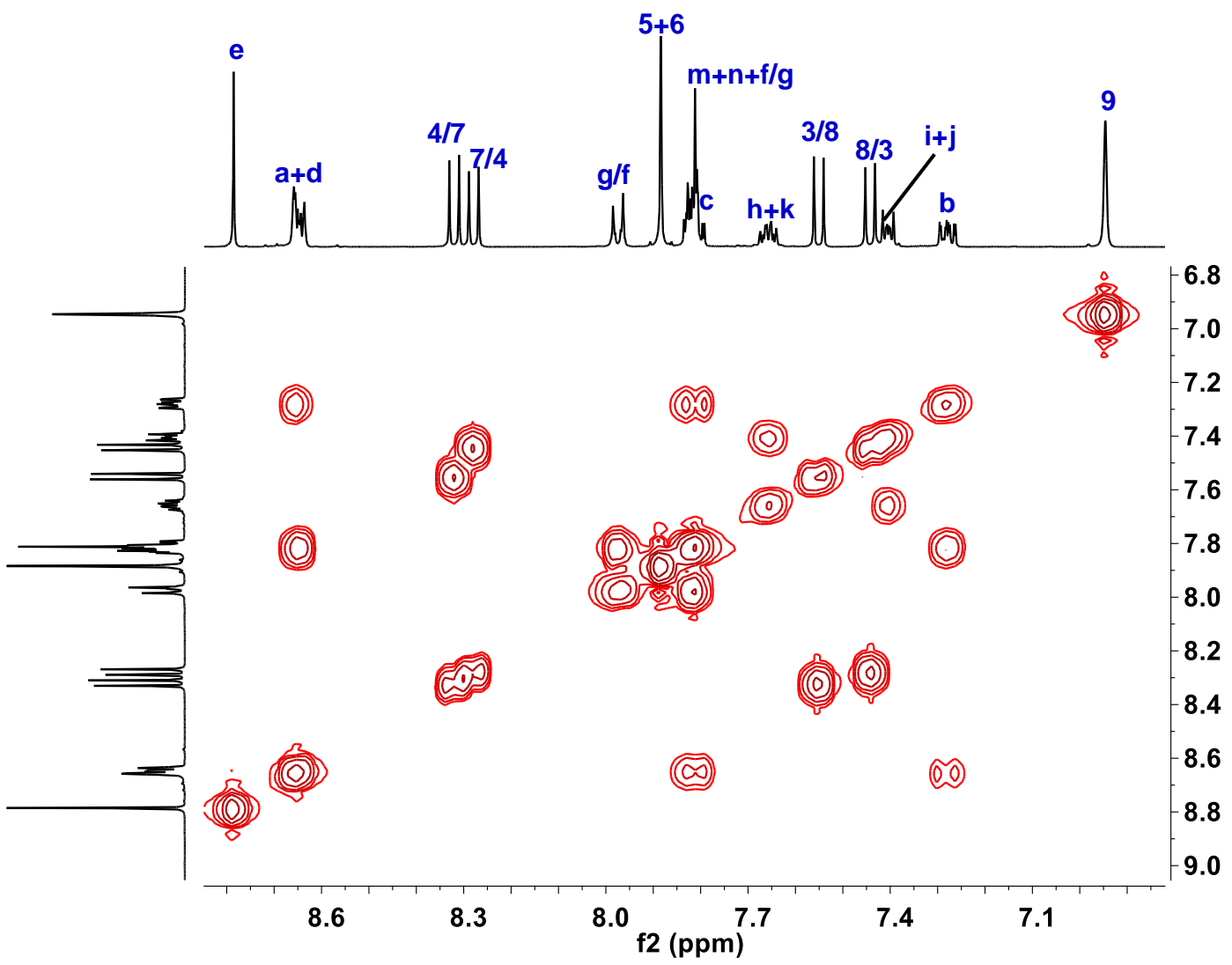

Figure S2. ${ }^{1} \mathrm{H}-{ }^{1} \mathrm{H}$ COSY spectrum of $\mathbf{1}$ in $\mathrm{CD}_{2} \mathrm{Cl}_{2}(400 \mathrm{MHz}, 298 \mathrm{~K})$. 


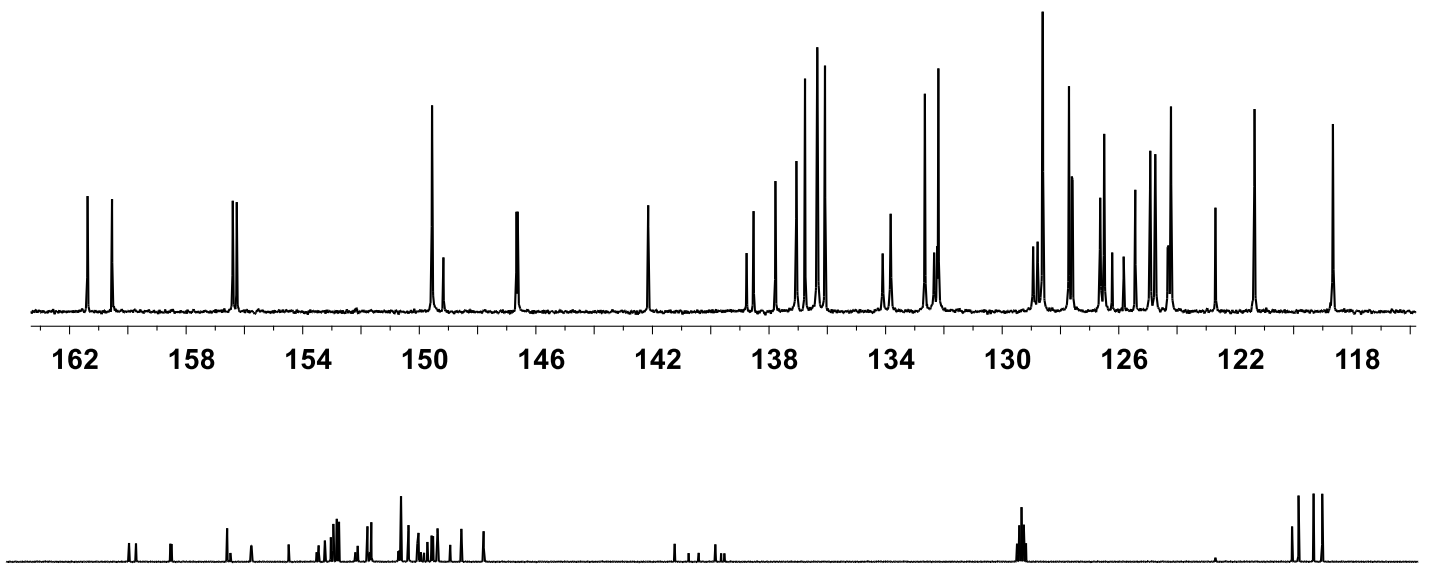

$\begin{array}{lllllllllllllllll}170 & 160 & 150 & 140 & 130 & 120 & 110 & 100 & 90 & 80 & 70 & 60 & 50 & 40 & 30 & 20 & 10\end{array}$

Figure S3. ${ }^{13} \mathrm{C}$ NMR spectrum of 1 in $\mathrm{CD}_{2} \mathrm{Cl}_{2}(100 \mathrm{MHz}, 298 \mathrm{~K})$.

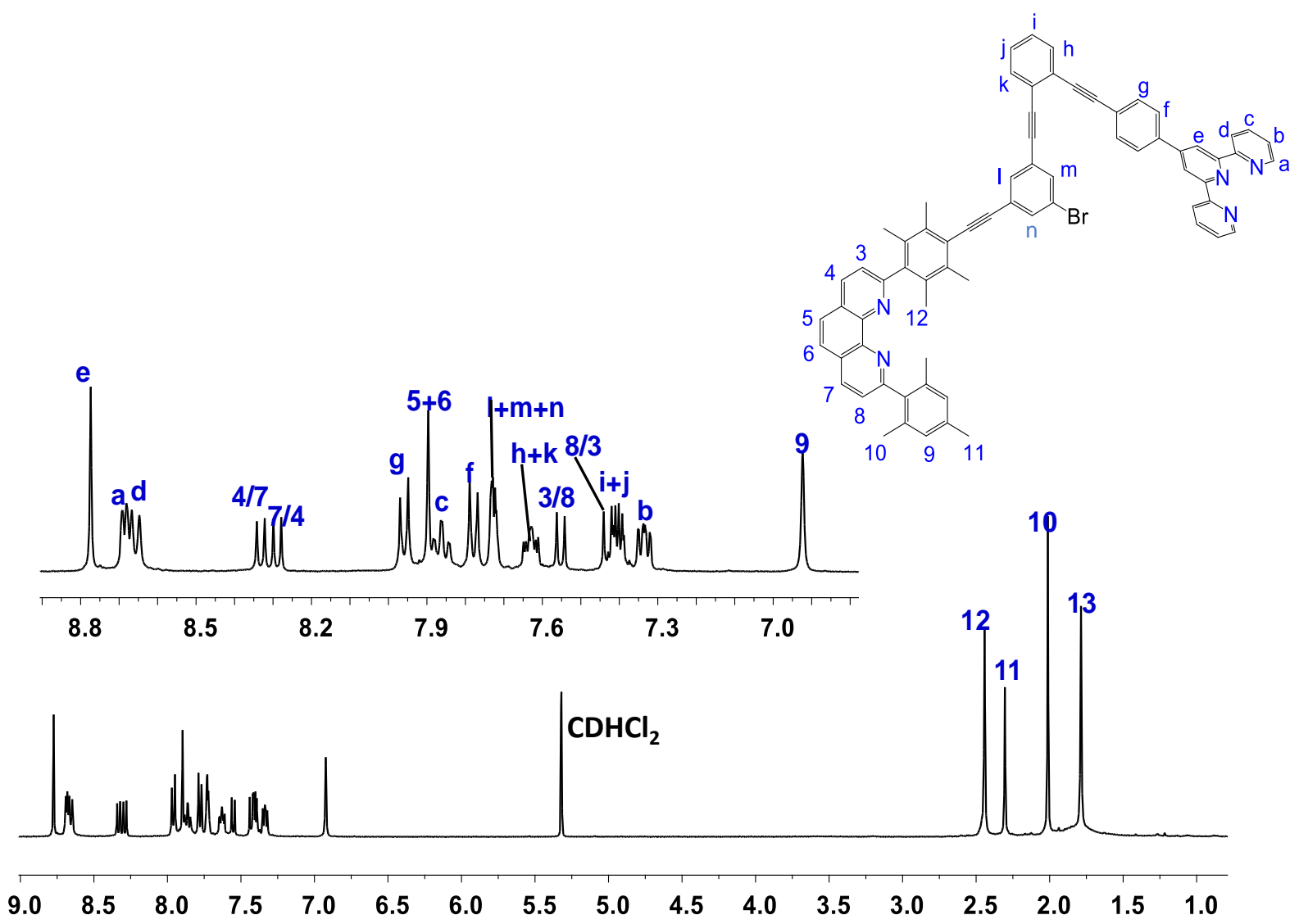

Figure S4. ${ }^{1} \mathrm{H}$ NMR spectrum of $\mathbf{1}$ ' in $\mathrm{CD}_{2} \mathrm{Cl}_{2}(400 \mathrm{MHz}, 298 \mathrm{~K})$. 


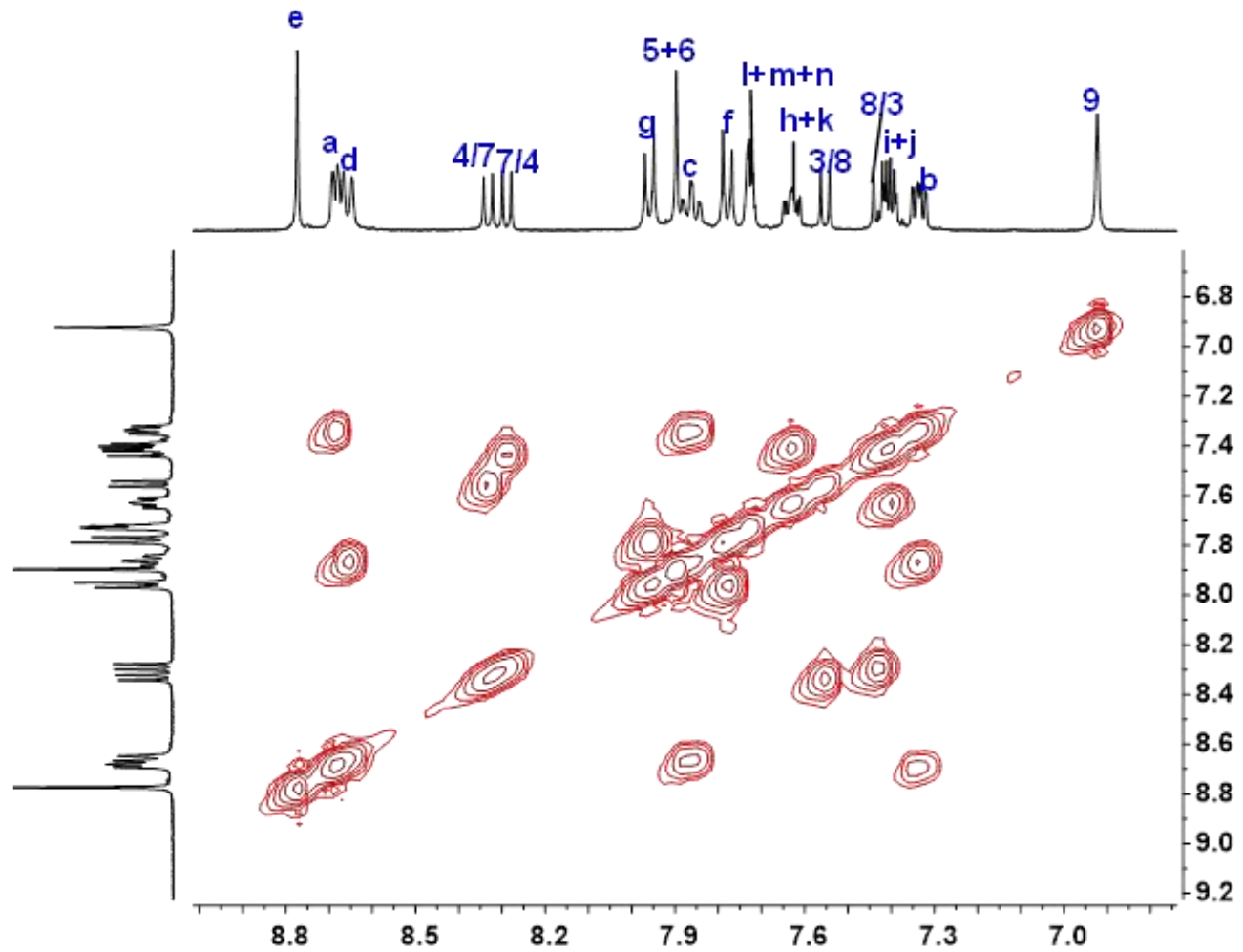

Figure S5. ${ }^{1} \mathrm{H}-{ }^{1} \mathrm{H}$ COSY spectrum of 1 ' in $\mathrm{CD}_{2} \mathrm{Cl}_{2}(400 \mathrm{MHz}, 298 \mathrm{~K})$.

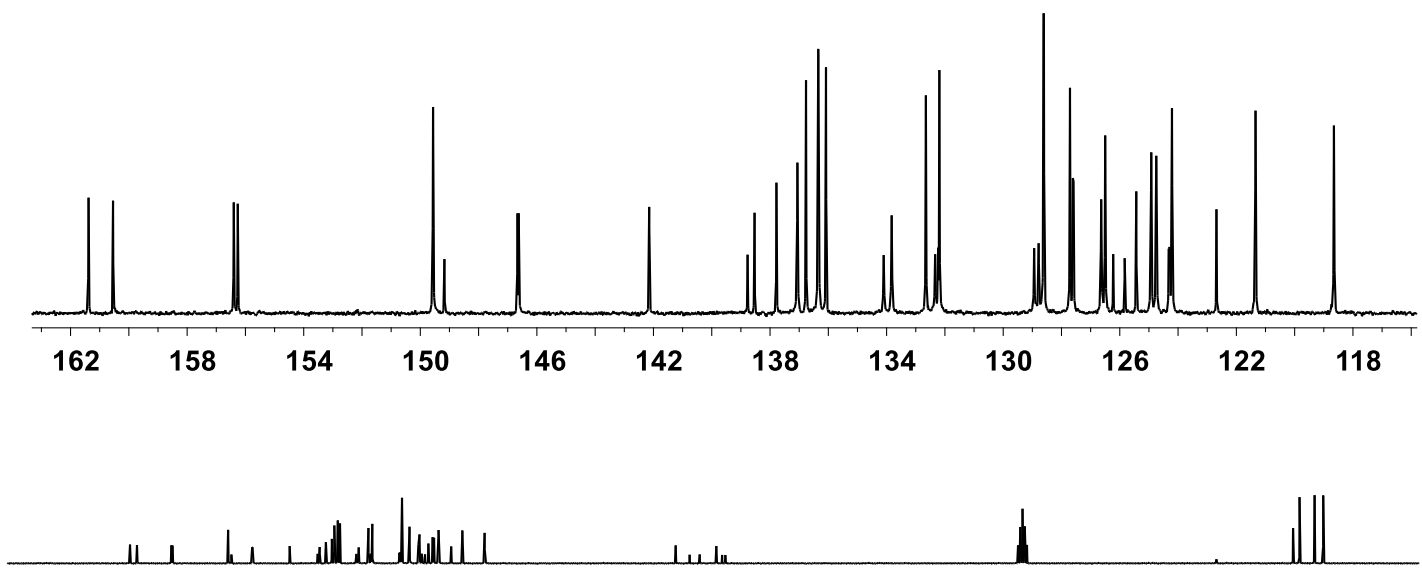

$\begin{array}{lllllllllllllllll}170 & 160 & 150 & 140 & 130 & 120 & 110 & 100 & 90 & 80 & 70 & 60 & 50 & 40 & 30 & 20 & 10\end{array}$

Figure S6. ${ }^{13} \mathrm{C}$ NMR spectrum of $\mathbf{1}$ ' in $\mathrm{CD}_{2} \mathrm{Cl}_{2}(100 \mathrm{MHz}, 298 \mathrm{~K})$. 


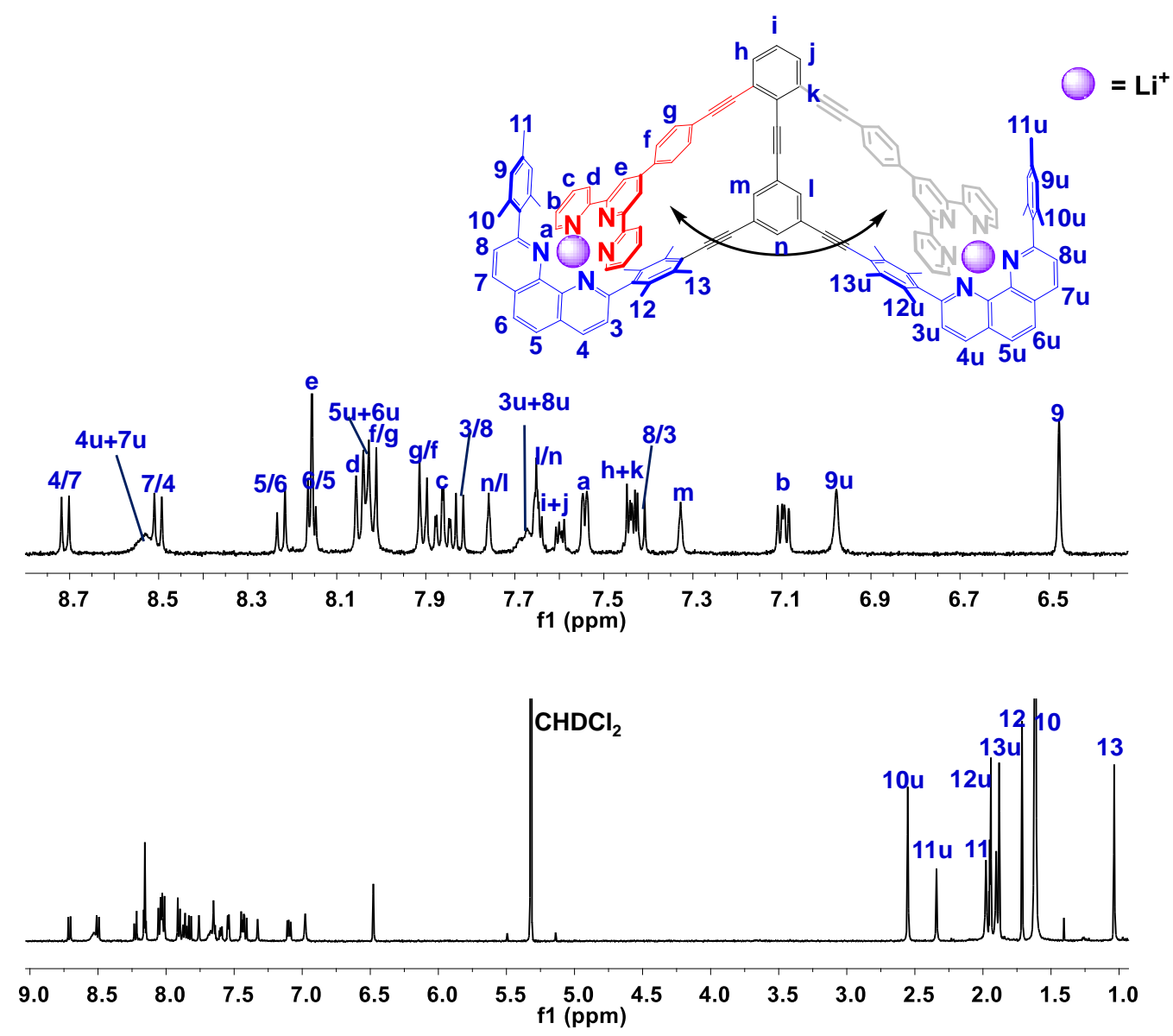

Figure S7. ${ }^{1} \mathrm{H}$ NMR spectrum of $\left[\mathrm{Li}_{2}(\mathbf{1})\right]^{2+}$ in $\mathrm{CD}_{2} \mathrm{Cl}_{2}: \mathrm{CD}_{3} \mathrm{CN}(5: 1)(500 \mathrm{MHz}, 298 \mathrm{~K})$.

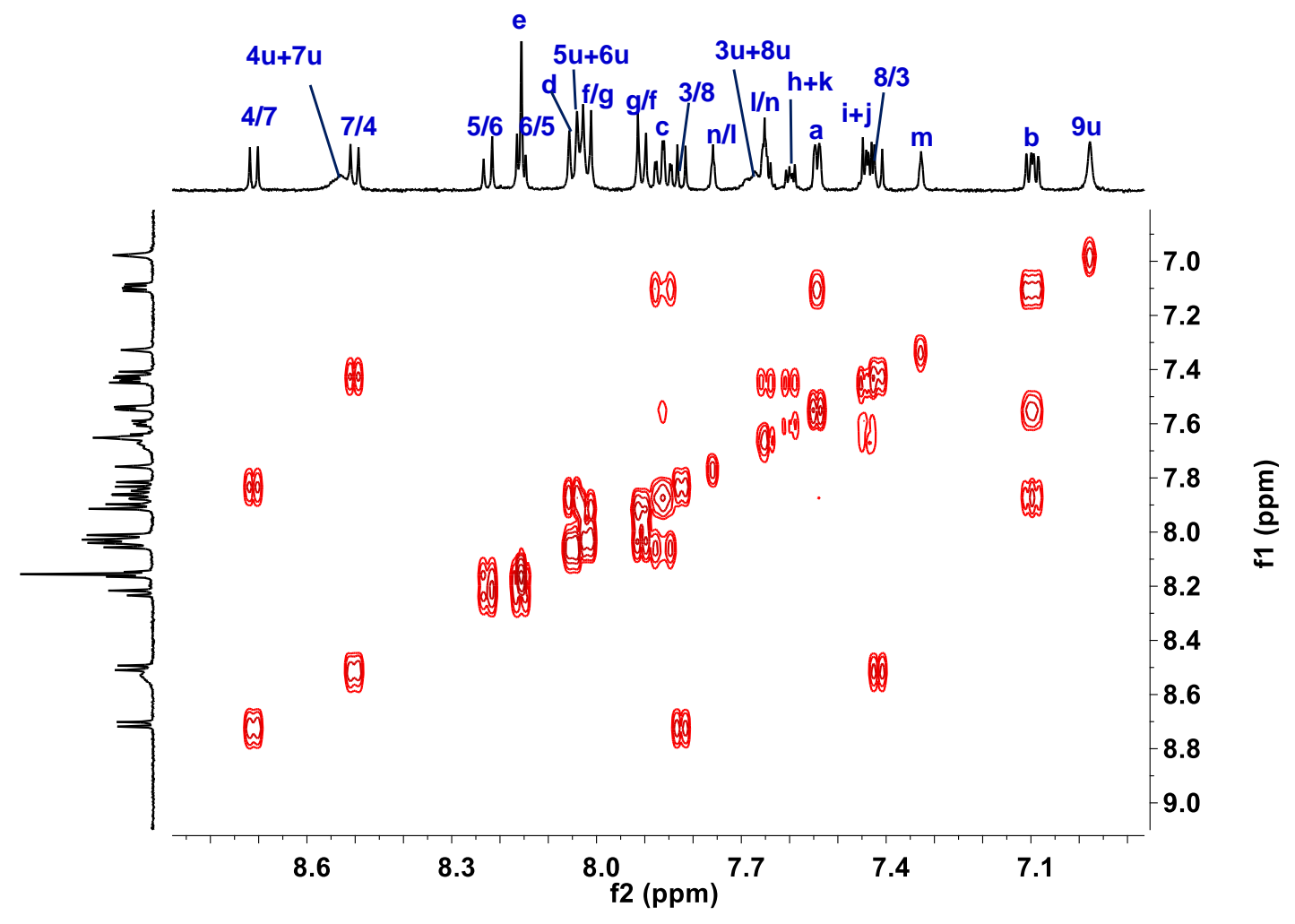

Figure S8. ${ }^{1} \mathrm{H}-{ }^{1} \mathrm{H}$ COSY spectrum of $\left[\mathrm{Li}_{2}(\mathbf{1})\right]^{2+}$ in $\mathrm{CD}_{2} \mathrm{Cl}_{2}: \mathrm{CD}_{3} \mathrm{CN}(5: 1)(500 \mathrm{MHz}, 298 \mathrm{~K})$. 

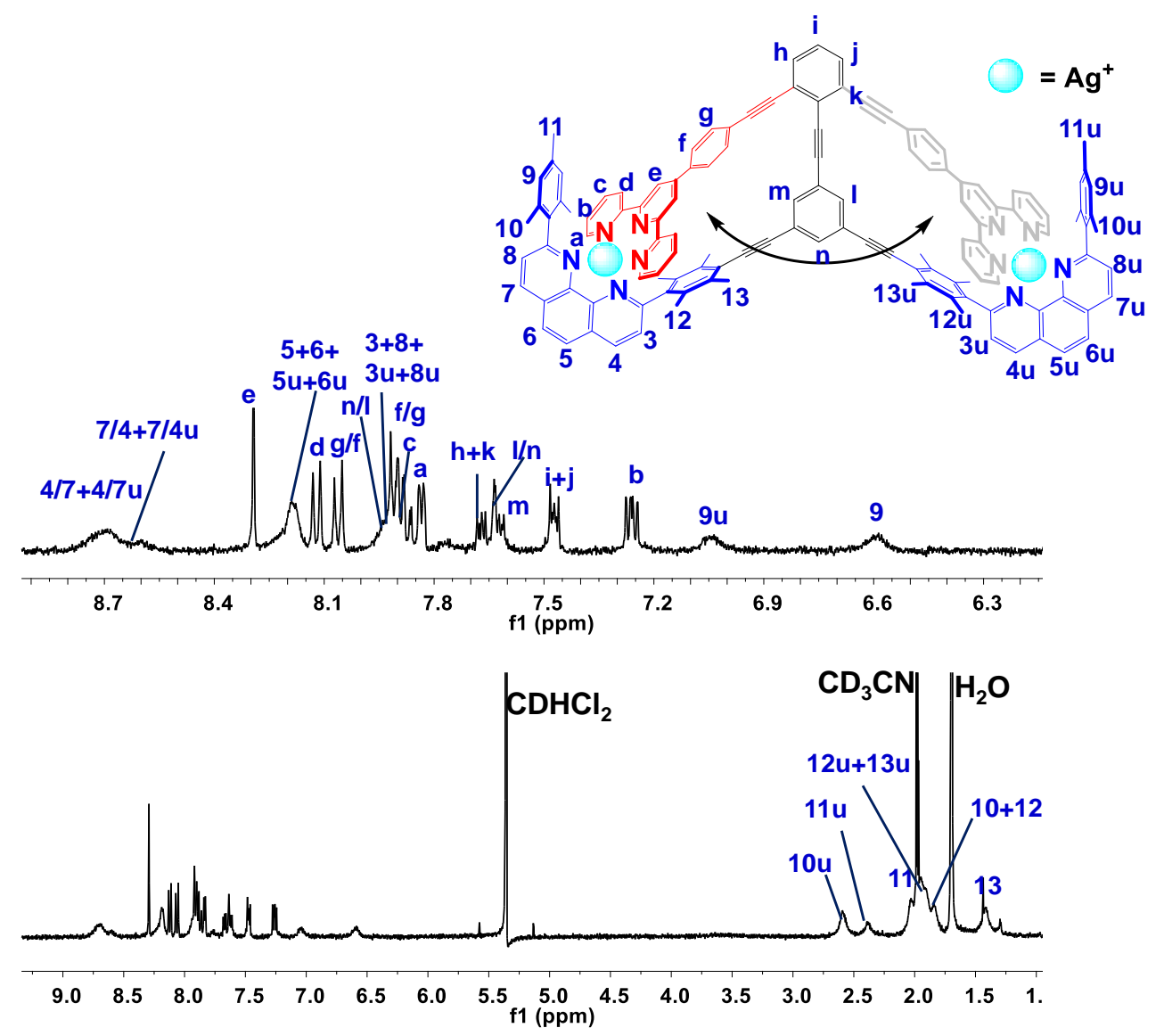

Figure S9. ${ }^{1} \mathrm{H}$ NMR spectrum of $\left[\mathrm{Ag}_{2}(\mathbf{1})\right]^{2+}$ in $\mathrm{CD}_{2} \mathrm{Cl}_{2}: \mathrm{CD}_{3} \mathrm{CN}(5: 1)(500 \mathrm{MHz}, 298 \mathrm{~K})$.

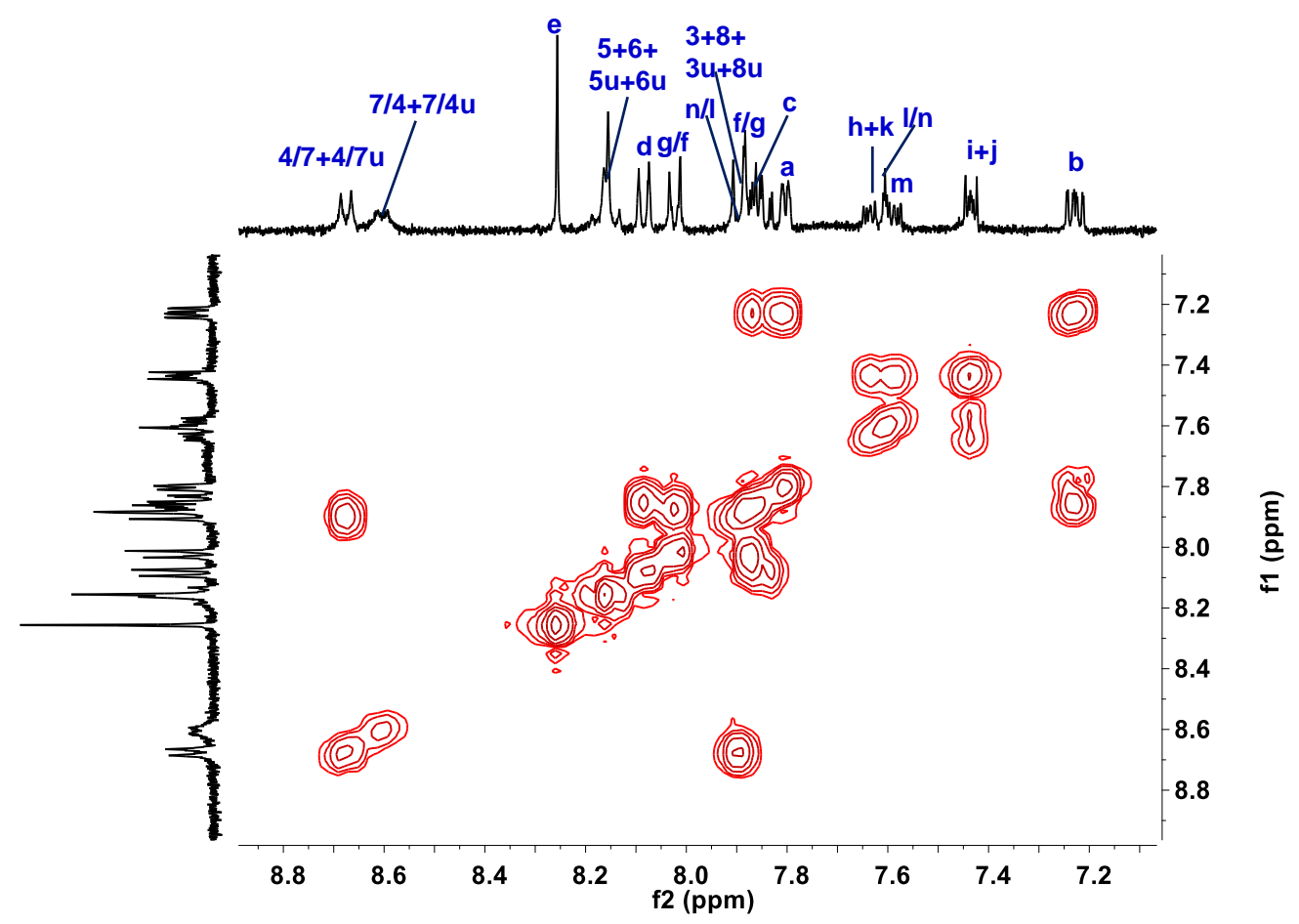

Figure S10. ${ }^{1} \mathrm{H}-{ }^{1} \mathrm{H}$ COSY spectrum of $\left[\mathrm{Ag}_{2}(\mathbf{1})\right]^{2+}$ in $\mathrm{CD}_{2} \mathrm{Cl}_{2}: \mathrm{CD}_{3} \mathrm{CN}(5: 1)(500 \mathrm{MHz}, 298 \mathrm{~K})$. 


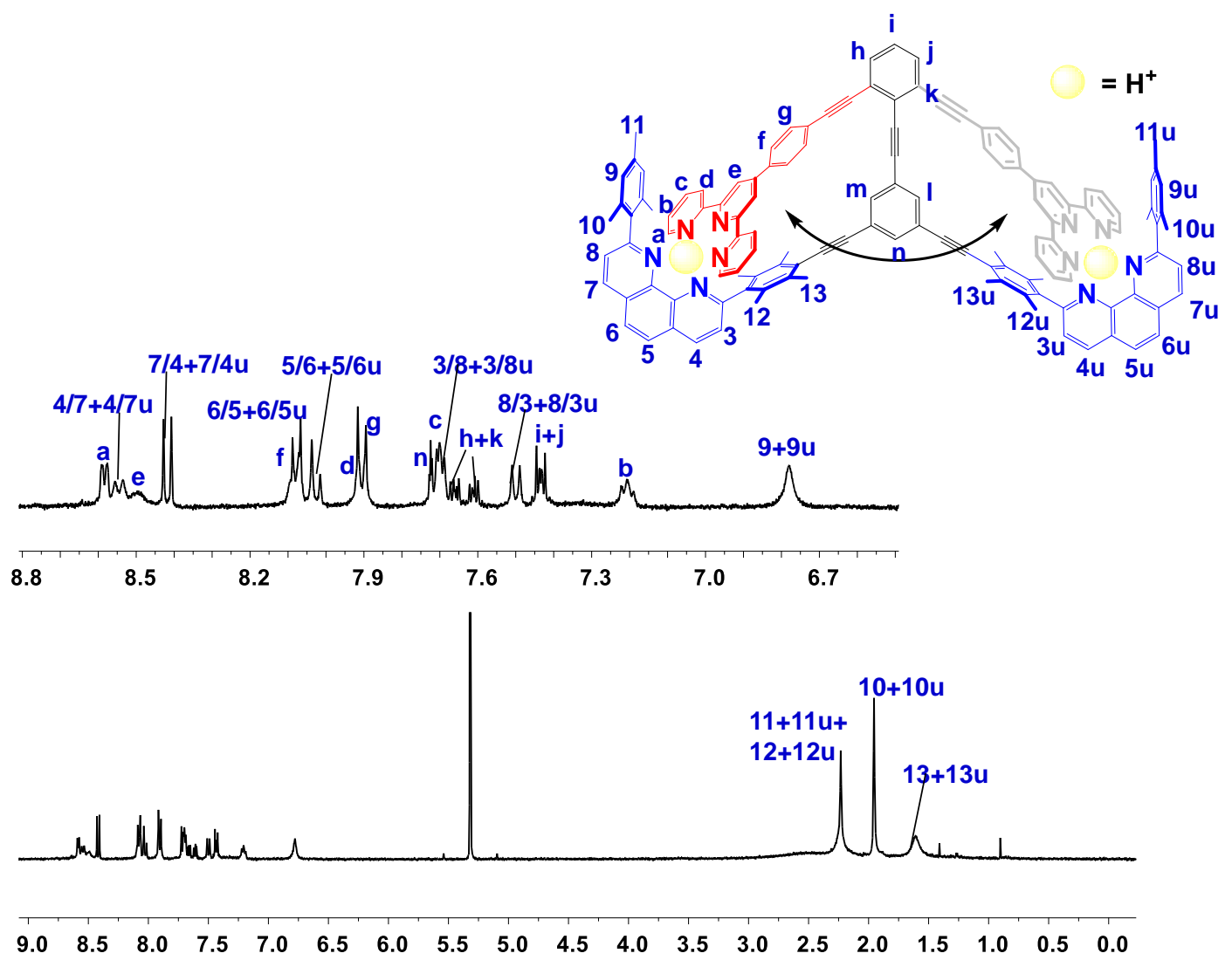

Figure S11. ${ }^{1} \mathrm{H}$ NMR spectrum of $\left[\mathrm{H}_{2}(\mathbf{1})\right]^{2+}$ in $\mathrm{CD}_{2} \mathrm{Cl}_{2}(500 \mathrm{MHz}, 298 \mathrm{~K})$.

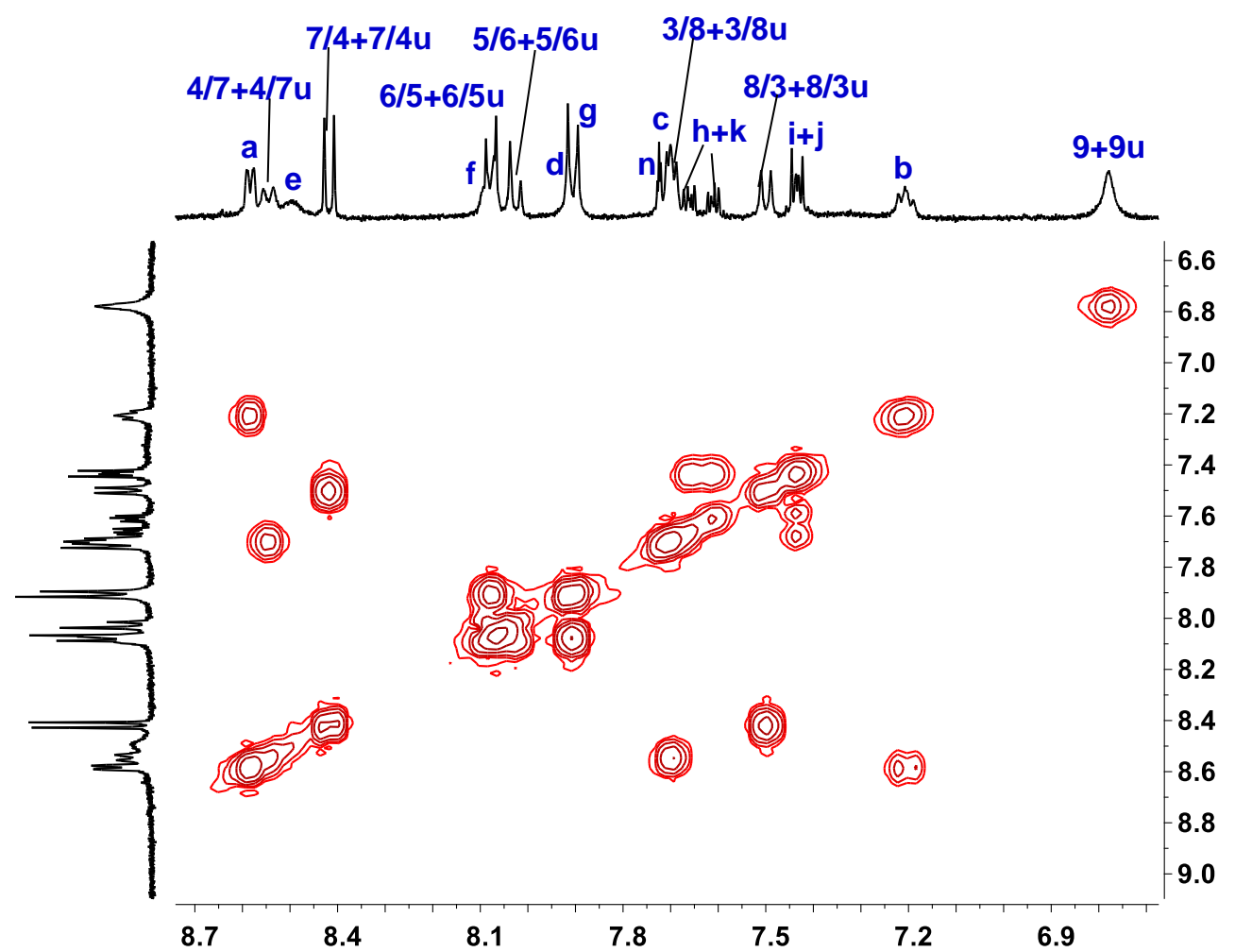

Figure S12. ${ }^{1} \mathrm{H}-{ }^{1} \mathrm{H}$ COSY spectrum of $\left[\mathrm{H}_{2}(\mathbf{1})\right]^{2+}$ in $\mathrm{CD}_{2} \mathrm{Cl}_{2}(500 \mathrm{MHz}, 298 \mathrm{~K})$. 


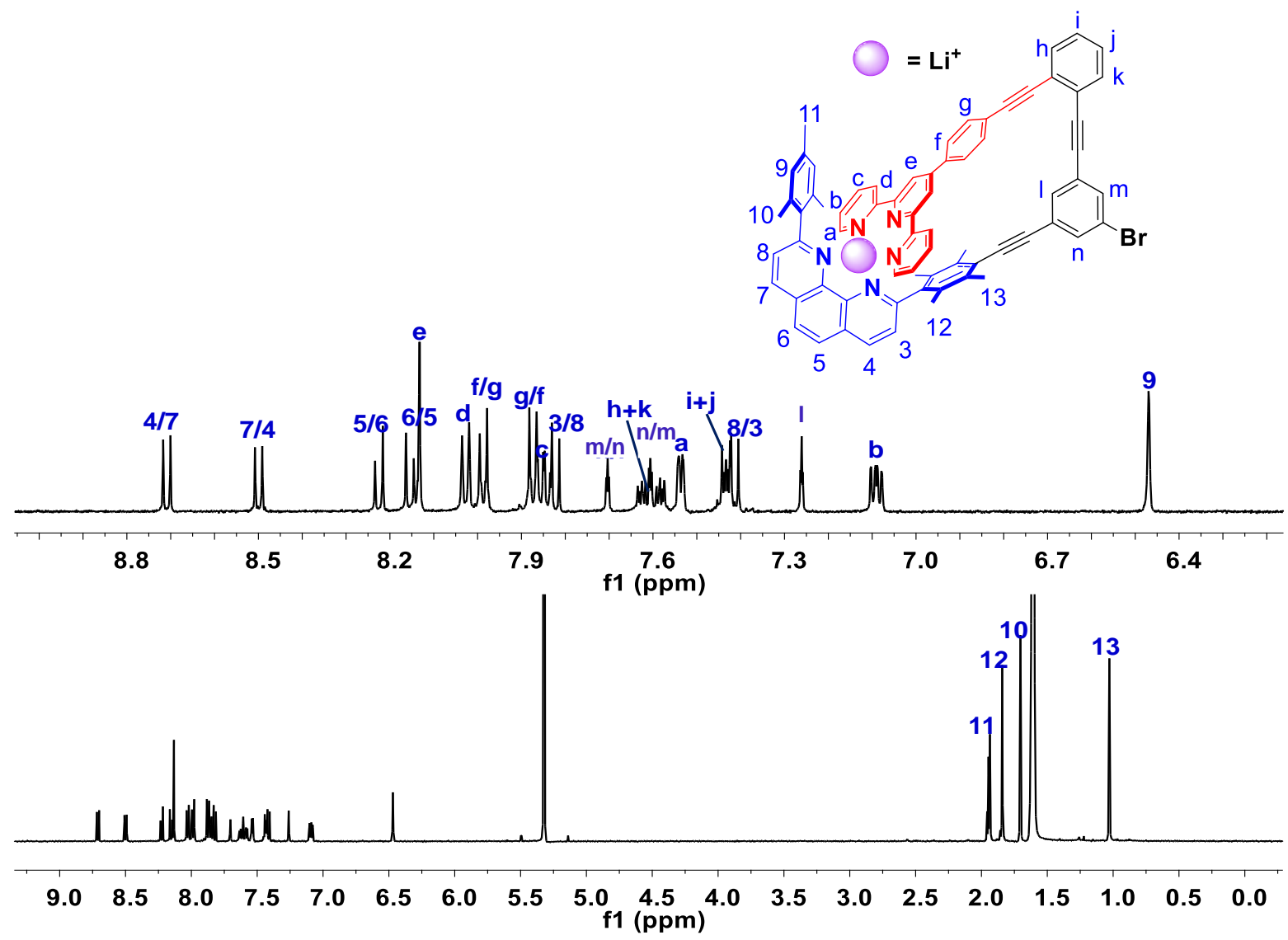

Figure S13. ${ }^{1} \mathrm{H}$ NMR spectrum of $\left[\mathrm{Li}\left(\mathbf{1}^{\prime}\right)\right]^{+}$in $\mathrm{CD}_{2} \mathrm{Cl}_{2}: \mathrm{CD}_{3} \mathrm{CN}(5: 1)(500 \mathrm{MHz}, 298 \mathrm{~K})$. 


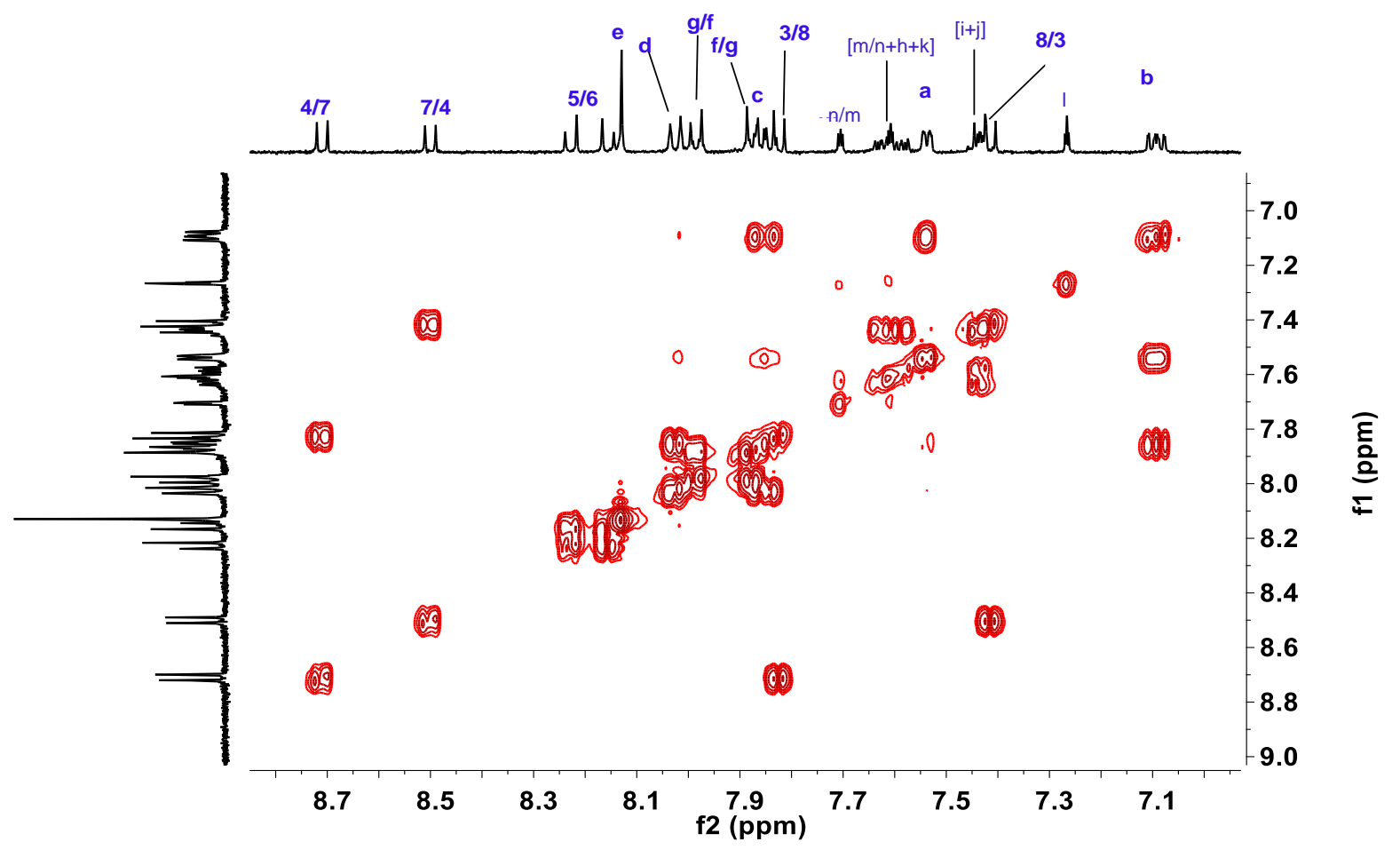

Figure S14. ${ }^{1} \mathrm{H}^{-1} \mathrm{H}$ COSY spectrum of $\left[\mathrm{Li}\left(\mathbf{1}^{\prime}\right)\right]^{+}$in $\mathrm{CD}_{2} \mathrm{Cl}_{2}: \mathrm{CD}_{3} \mathrm{CN}(5: 1)(500 \mathrm{MHz}, 298 \mathrm{~K})$.
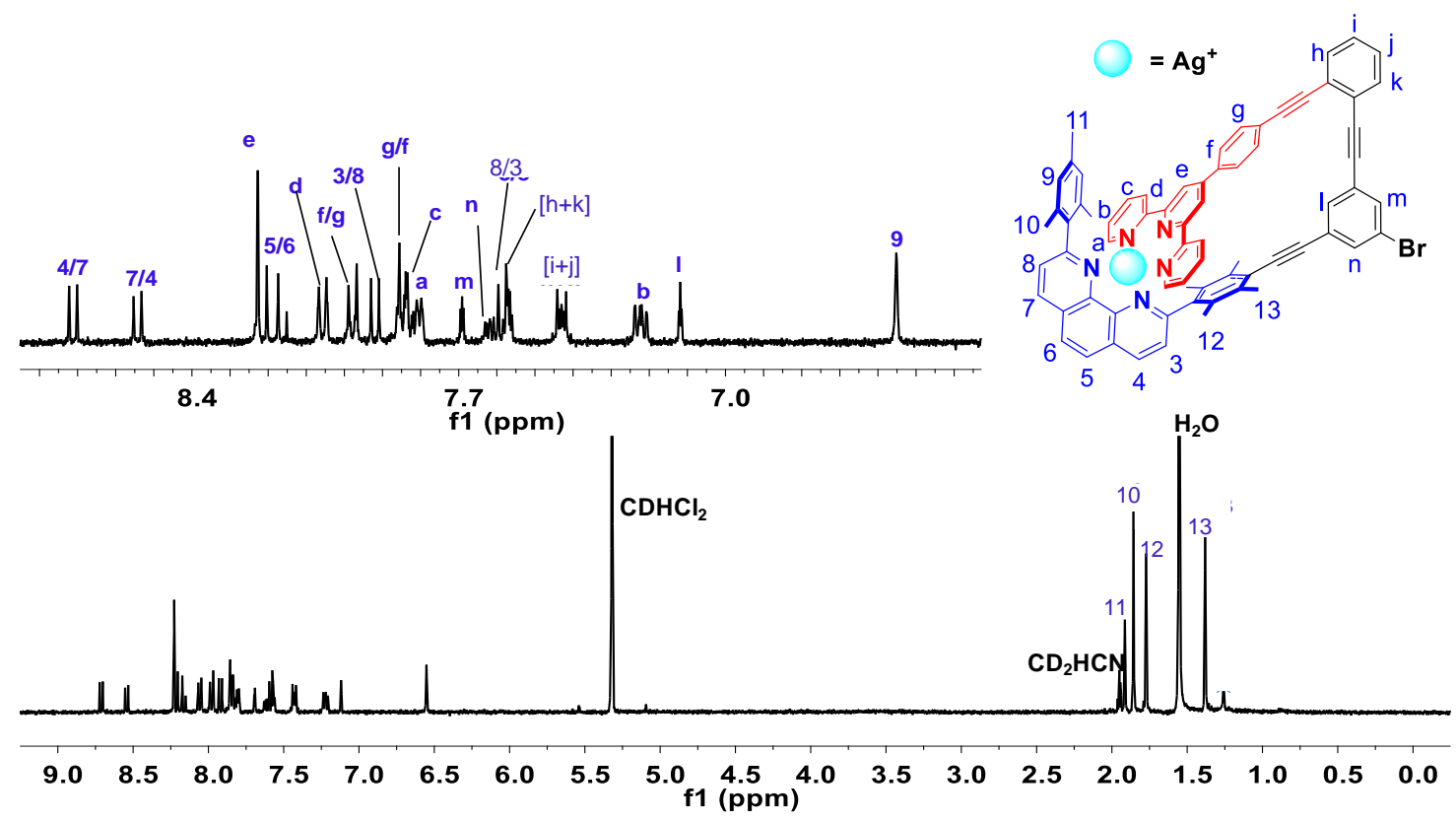

Figure S15. ${ }^{1} \mathrm{H}$ NMR spectrum of $\left[\mathrm{Ag}\left(\mathbf{1}^{\prime}\right)\right]^{+}$in $\mathrm{CD}_{2} \mathrm{Cl}_{2}: \mathrm{CD}_{3} \mathrm{CN}(5: 1)(500 \mathrm{MHz}, 298 \mathrm{~K})$. 


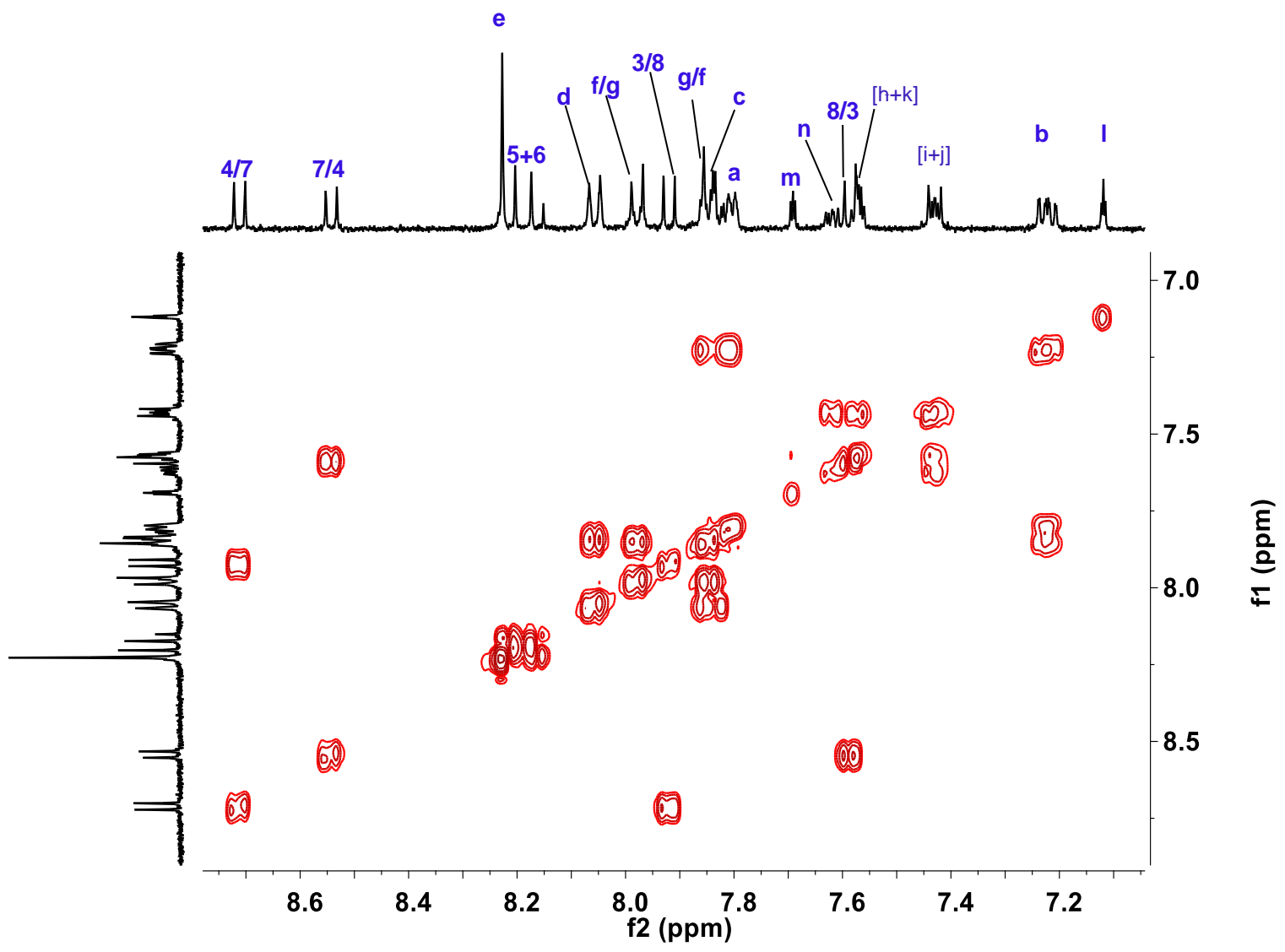

Figure S16. ${ }^{1} \mathrm{H}^{-1} \mathrm{H}$ COSY spectrum of $\left[\mathrm{Ag}\left(\mathbf{1}^{\prime}\right)\right]^{+}$in $\mathrm{CD}_{2} \mathrm{Cl}_{2}: \mathrm{CD}_{3} \mathrm{CN}(5: 1)(500 \mathrm{MHz}, 298 \mathrm{~K})$.
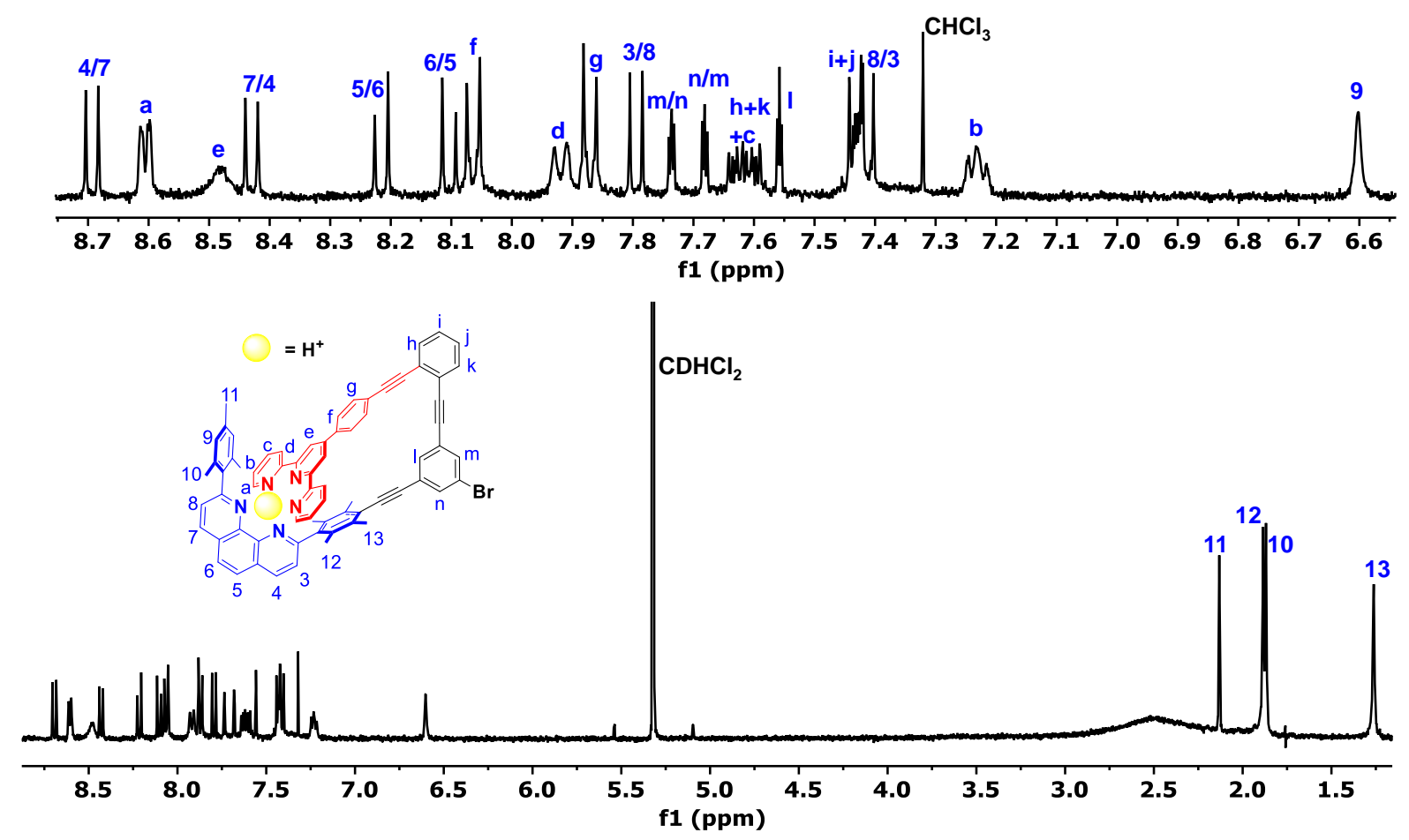

Figure S17. ${ }^{1} \mathrm{H}$ NMR spectrum of $\left[\mathrm{H}\left(\mathbf{1}^{\prime}\right)\right]^{+}$in $\mathrm{CD}_{2} \mathrm{Cl}_{2}(500 \mathrm{MHz}, 298 \mathrm{~K})$. 

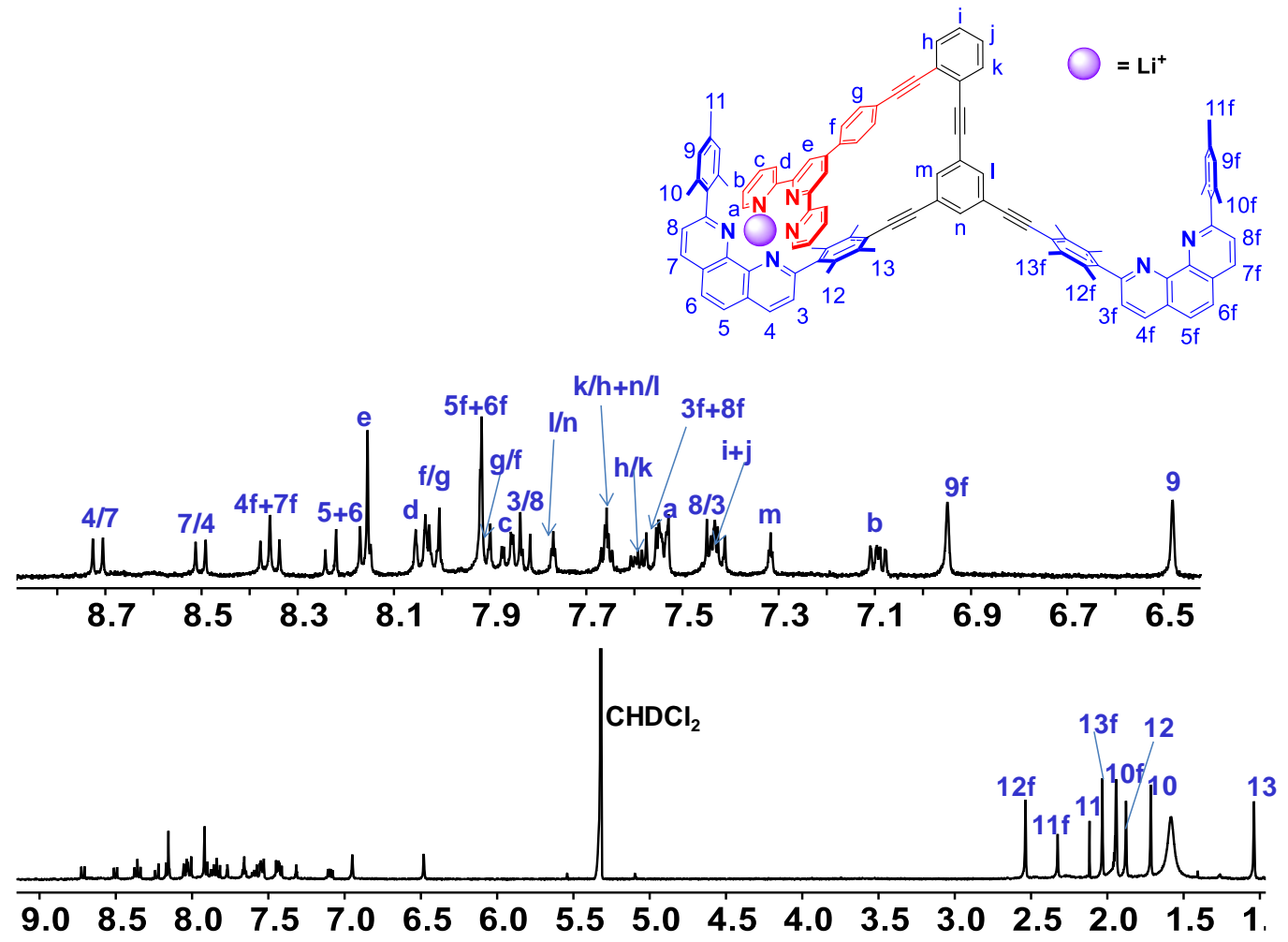

Figure S18. ${ }^{1} \mathrm{H}$ NMR spectrum of $[\mathrm{Li}(\mathbf{1})]^{+}$in $\mathrm{CD}_{2} \mathrm{Cl}_{2}: \mathrm{CD}_{3} \mathrm{CN}(5: 1)(500 \mathrm{MHz}, 298 \mathrm{~K})$.

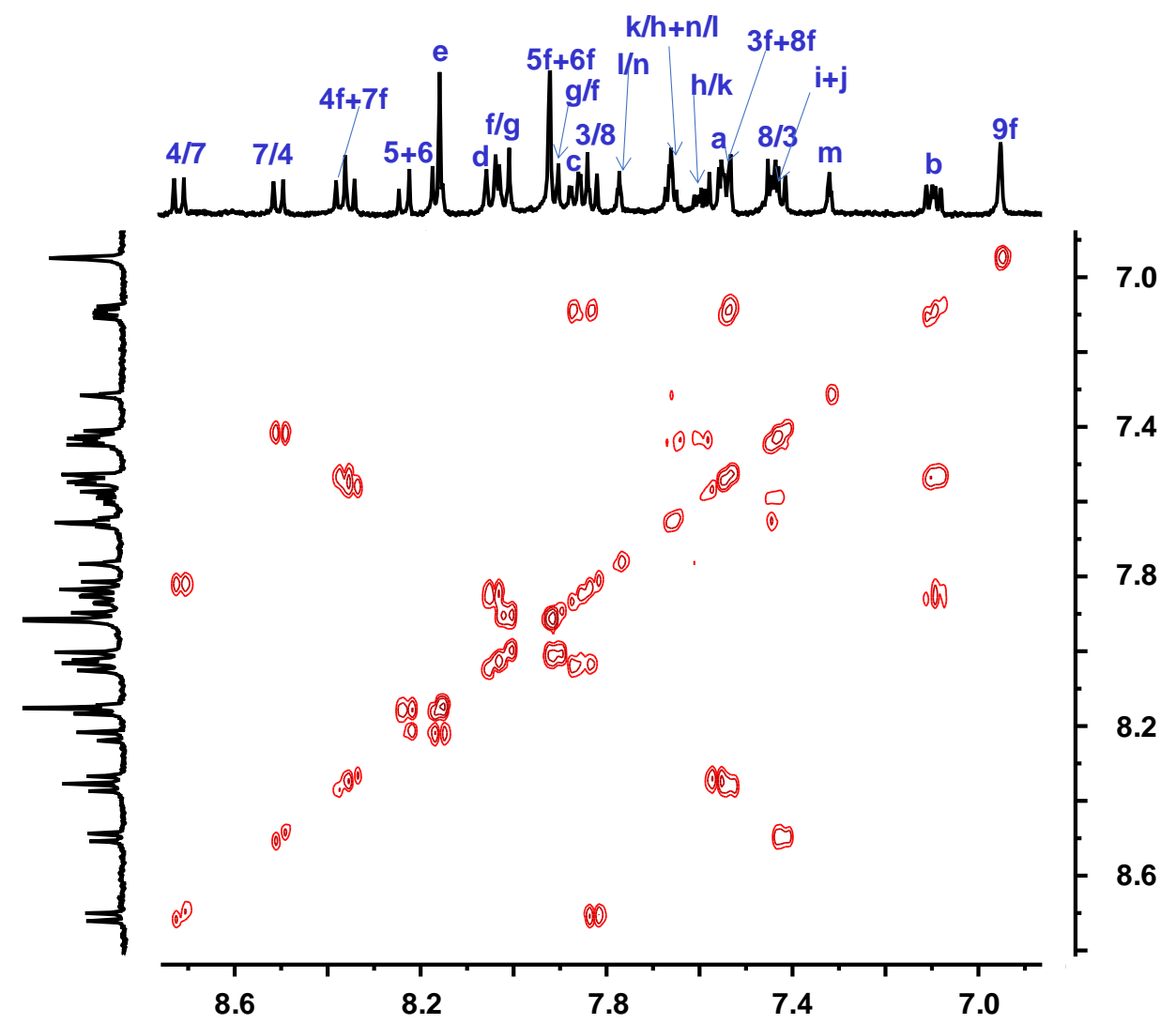

Figure 19. ${ }^{1} \mathrm{H}-{ }^{1} \mathrm{H}$ COSY spectrum of $[\mathrm{Li}(\mathbf{1})]^{+}$in $\mathrm{CD}_{2} \mathrm{Cl}_{2}: \mathrm{CD}_{3} \mathrm{CN}(5: 1)(500 \mathrm{MHz}, 298 \mathrm{~K})$. 


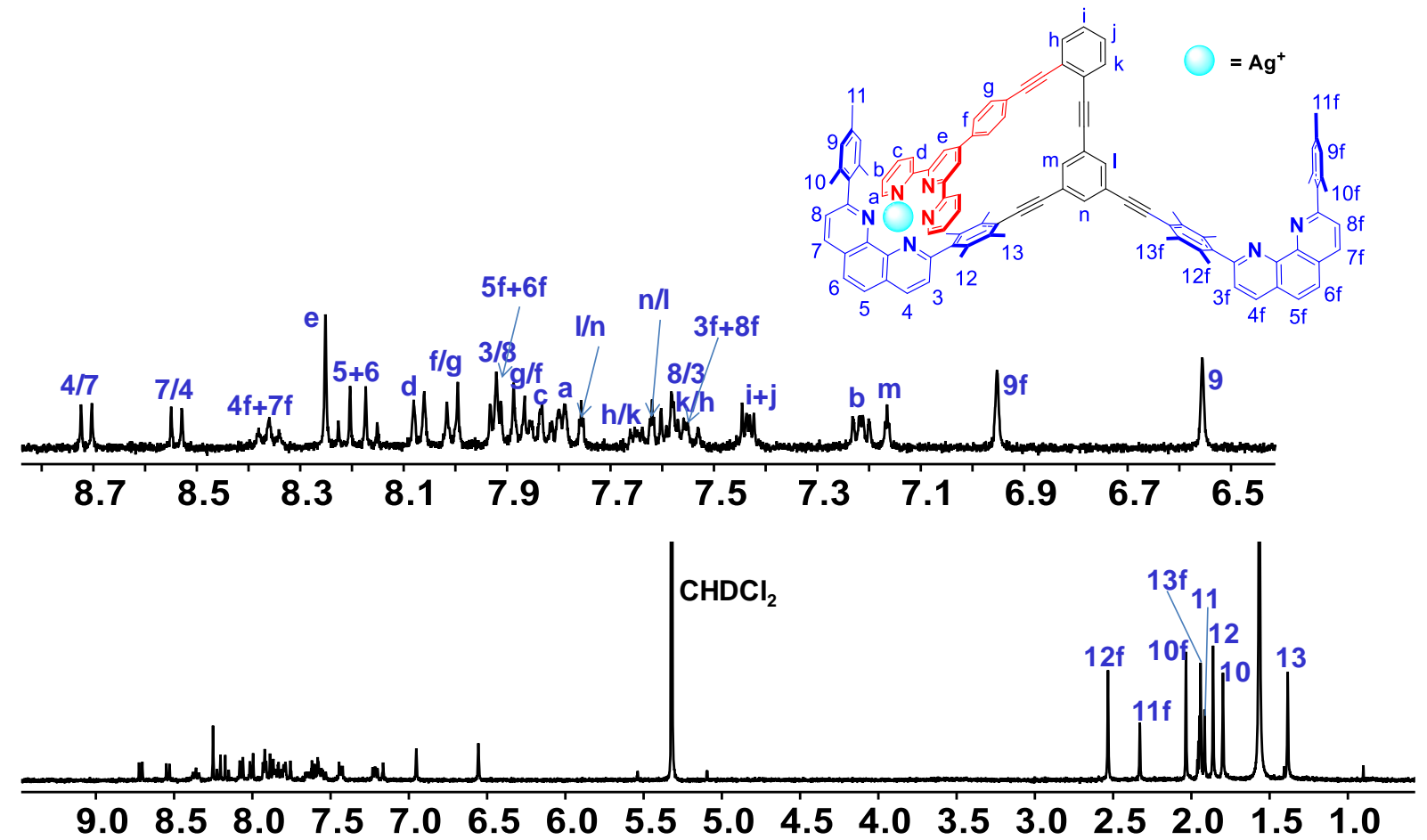

Figure S20. ${ }^{1} \mathrm{H}$ NMR spectrum of $[\mathrm{Ag}(\mathbf{1})]^{+}$in $\mathrm{CD}_{2} \mathrm{Cl}_{2}: \mathrm{CD}_{3} \mathrm{CN}(5: 1)(500 \mathrm{MHz}, 298 \mathrm{~K})$.

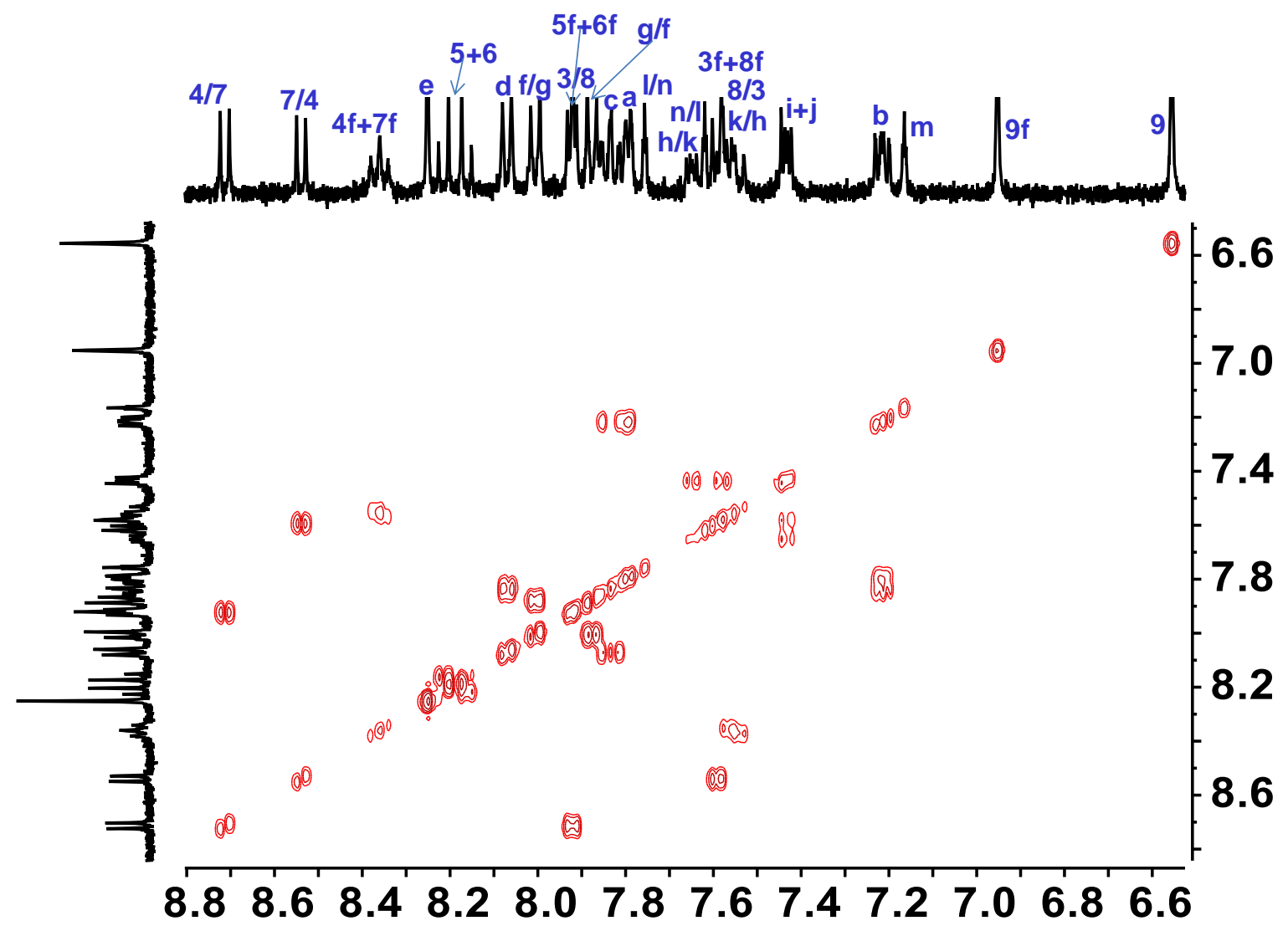

Figure S21. ${ }^{1} \mathrm{H}-{ }^{1} \mathrm{H}$ COSY spectrum of $[\mathrm{Ag}(\mathbf{1})]^{+}$in $\mathrm{CD}_{2} \mathrm{Cl}_{2}: \mathrm{CD}_{3} \mathrm{CN}(5: 1)(500 \mathrm{MHz}, 298 \mathrm{~K})$. 

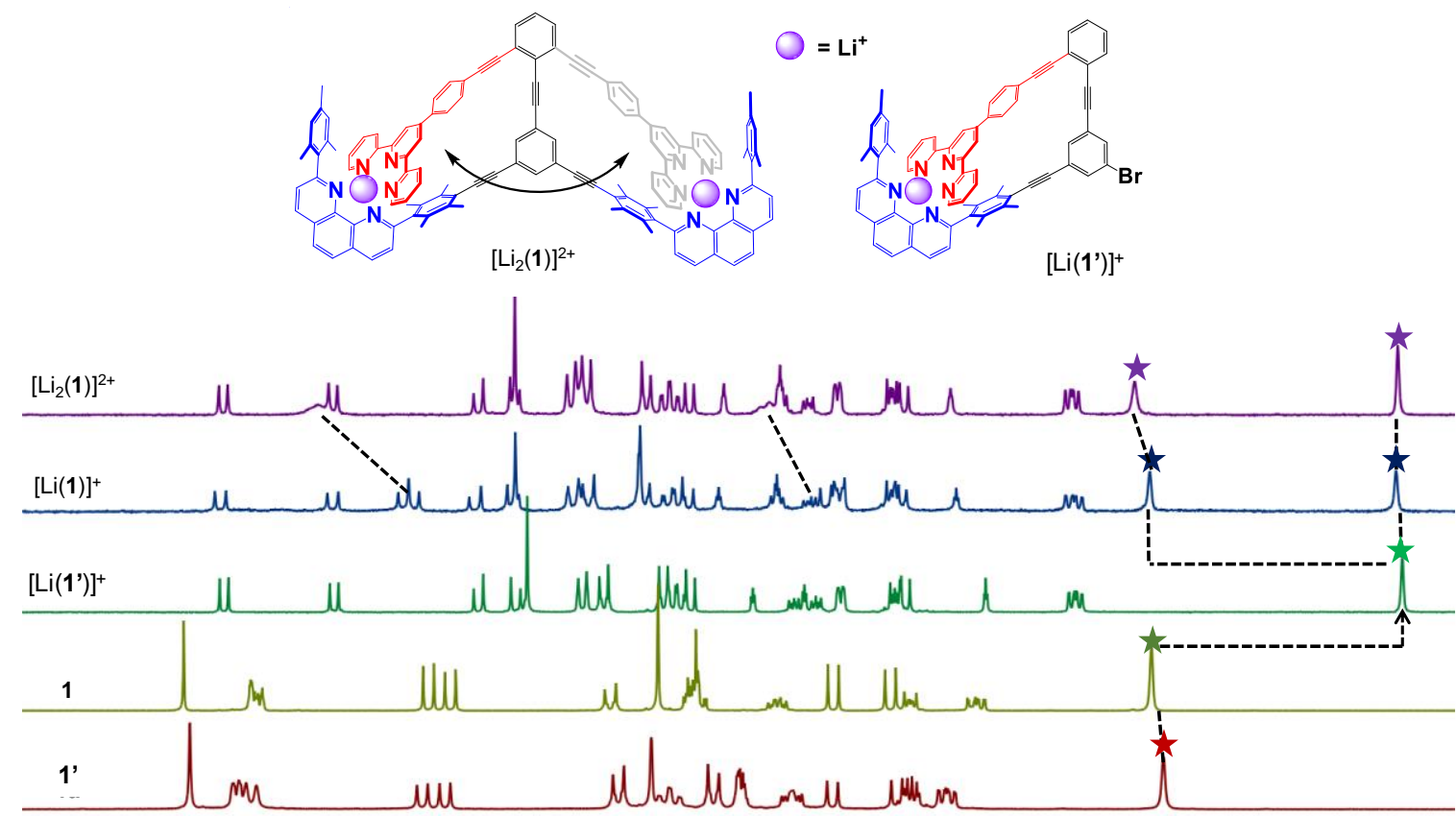

$\begin{array}{lllllllllllllllllllllllllll}9.0 & 8.9 & 8.8 & 8.7 & 8.6 & 8.5 & 8.4 & 8.3 & 8.2 & 8.1 & 8.0 & 7.9 & 7.8 & 7.7 & 7.6 & 7.5 & 7.4 & 7.3 & 7.2 & 7.1 & 7.0 & 6.9 & 6.8 & 6.7 & 6.6 & 6.5 & 6.4\end{array}$ f1 (ppm)

Figure S22. Partial ${ }^{1} \mathrm{H}$ NMR (400 MHz, $298 \mathrm{~K}$ ) of 1', 1 in $\mathrm{CD}_{2} \mathrm{Cl}_{2}$ and $\left[\operatorname{Li}\left(\mathbf{1}^{\text {' }}\right)\right]^{+},[\operatorname{Li}(\mathbf{1})]^{+}$, $\left[\mathrm{Li}_{2}(\mathbf{1})\right]^{2+}$ in $\mathrm{CD}_{2} \mathrm{Cl}_{2}: \mathrm{CD}_{3} \mathrm{CN}(5: 1)$. Due to the single set of mesityl protons (asterisk) in $\left[\operatorname{Li}\left(\mathbf{1}^{\prime}\right)\right]^{+}$there is magnetic equivalence for these protons in the HETTAP binding site. Furthermore, the two separate signals for the mesityl protons (asterisk) in $[\mathrm{Li}(\mathbf{1})]^{+}$and $\left[\mathrm{Li}_{2}(\mathbf{1})\right]^{2+}$ indicate slow rotational exchange. 

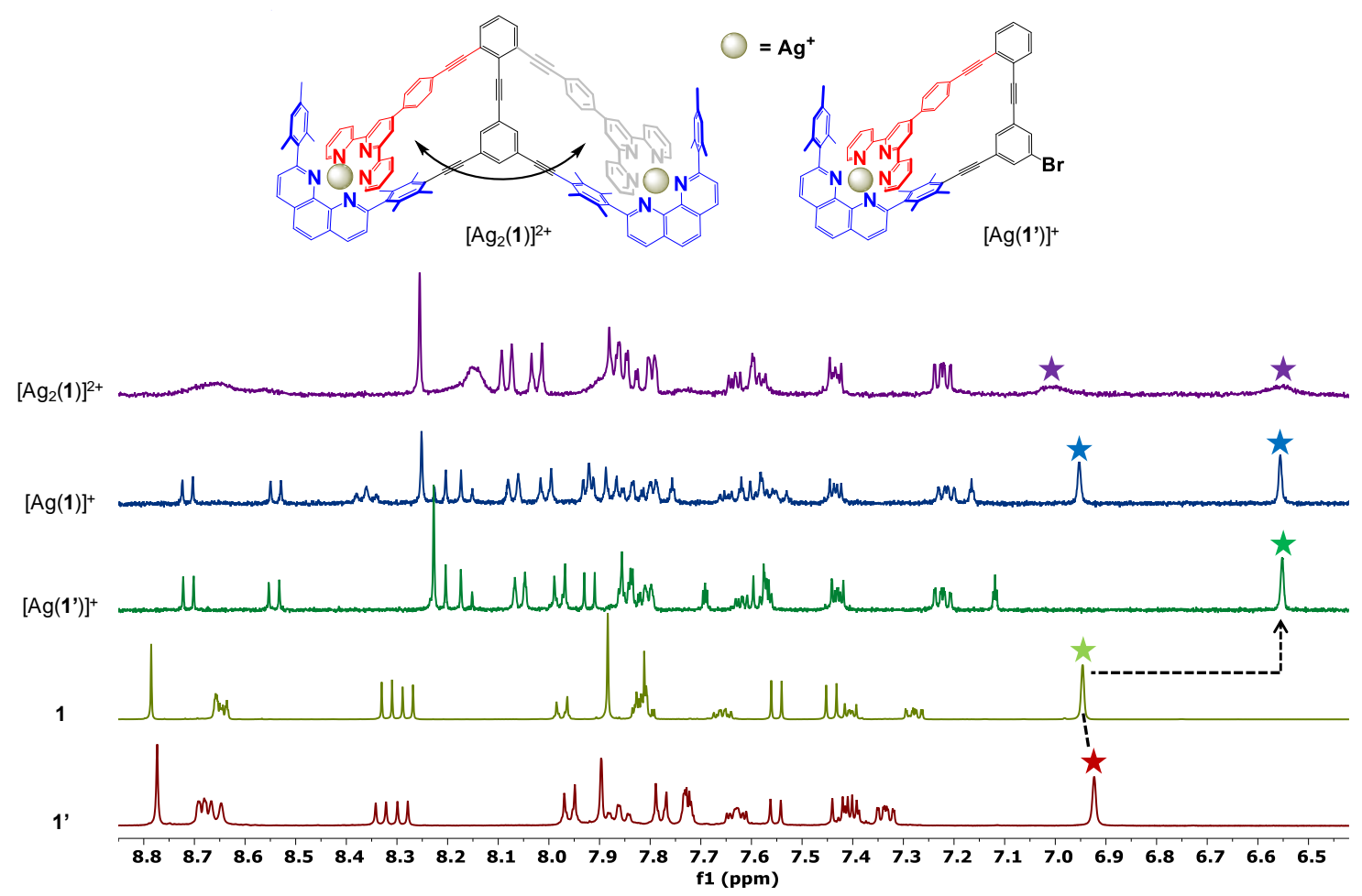

Figure S23. Partial ${ }^{1} \mathrm{H}$ NMR (400 MHz, $\left.298 \mathrm{~K}\right)$ of 1', $\mathbf{1}$ in $\mathrm{CD}_{2} \mathrm{Cl}_{2}$ and $\left[\mathrm{Ag}\left(\mathbf{1}^{\prime}\right)\right]^{+},[\mathrm{Ag}(\mathbf{1})]^{+}$, $\left[\mathrm{Ag}_{2}(\mathbf{1})\right]^{2+}$ in $\mathrm{CD}_{2} \mathrm{Cl}_{2}: \mathrm{CD}_{3} \mathrm{CN}$ (5:1). Due to the single set (asterisk) of mesityl protons in $\left[\operatorname{Ag}\left(\mathbf{1}^{\prime}\right)\right]^{+}$there is magnetic equivalence for these protons in the HETTAP binding site. Furthermore, the two separate signals for the mesityl protons (asterisk) in $[\operatorname{Ag}(\mathbf{1})]^{+}$and $\left[\mathrm{Ag}_{2}(\mathbf{1})\right]^{2+}$ indicate slow rotational exchange. 

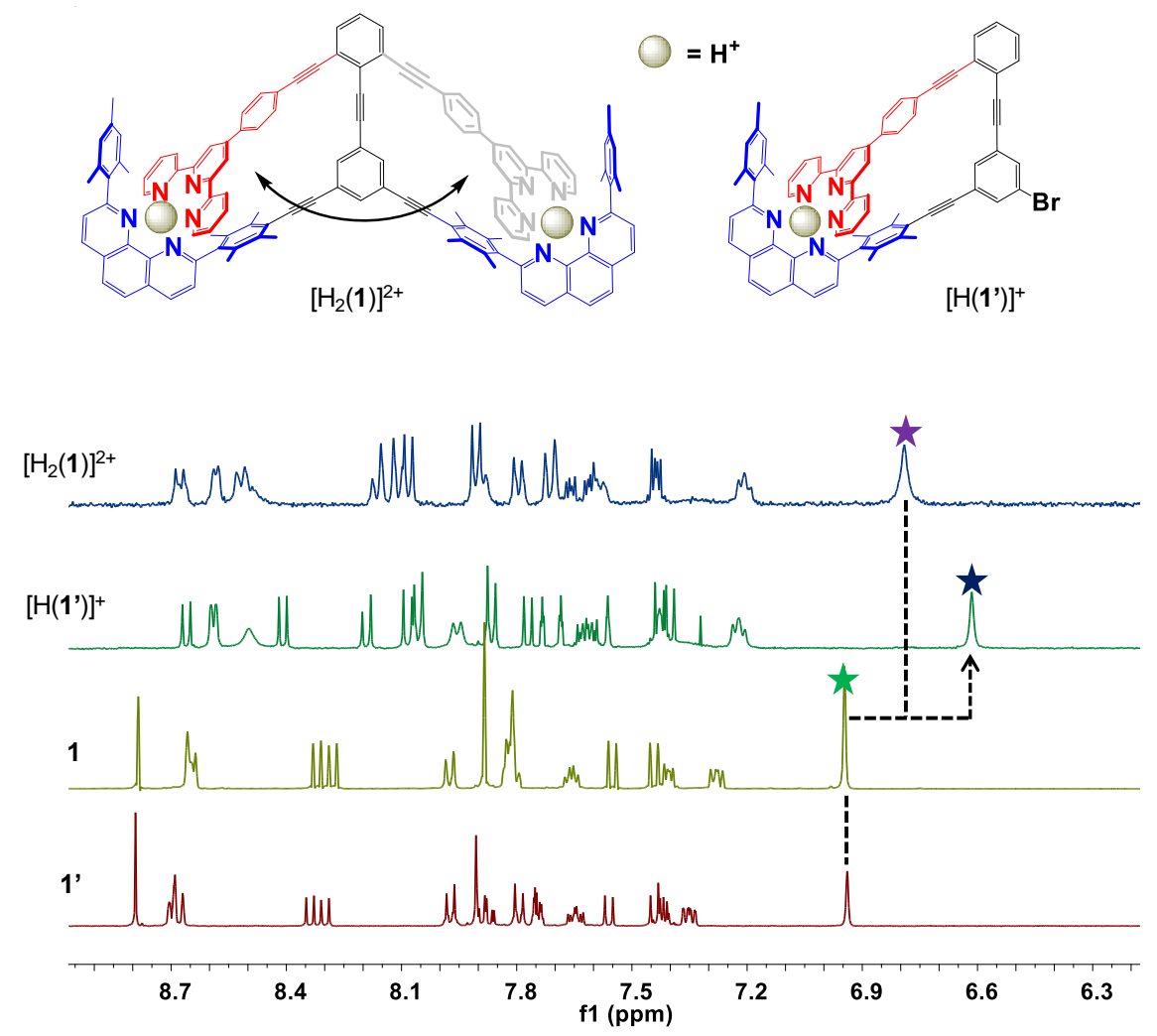

Figure S24. Partial ${ }^{1} \mathrm{H}$ NMR $(400 \mathrm{MHz}, 298 \mathrm{~K})$ of $\mathbf{1}^{\prime}, \mathbf{1},\left[\mathrm{H}\left(\mathbf{1}^{\prime}\right)\right]^{+}$and $\left[\mathrm{H}_{2}(\mathbf{1})\right]^{2+}$ in $\mathrm{CD}_{2} \mathrm{Cl}_{2}$. The single signal for the mesityl protons (asterisk) suggests fast rotation.

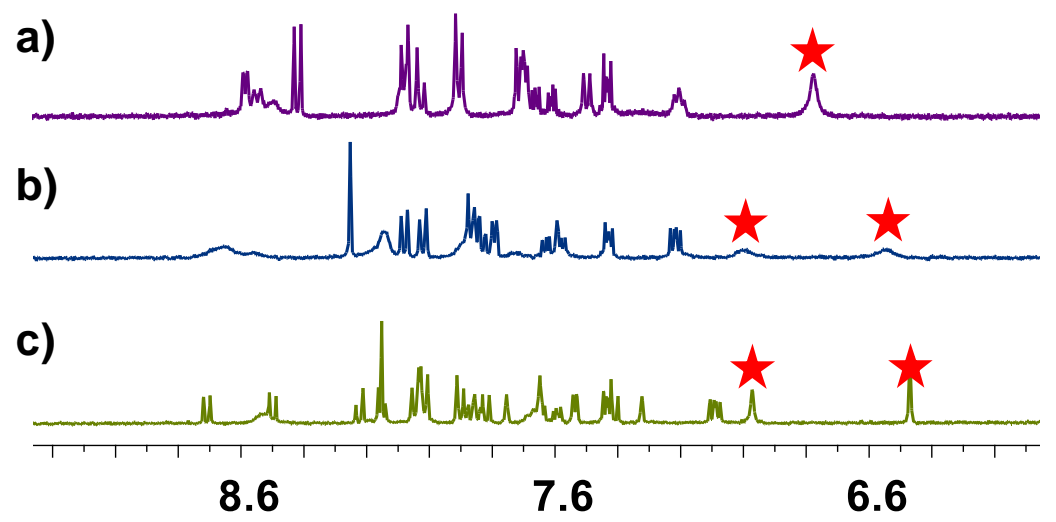

Figure S25. Partial ${ }^{1} \mathrm{H}$ NMR (400 MHz, $298 \mathrm{~K}$ ) of (a) $\left[\mathrm{H}_{2}(\mathbf{1})\right]^{2+}$ in $\mathrm{CD}_{2} \mathrm{Cl}_{2}$; (b) $\left[\mathrm{Ag}_{2}(\mathbf{1})\right]^{2+}$, (c) $\left[\mathrm{Li}_{2}(\mathbf{1})\right]^{2+}$ in $\mathrm{CD}_{2} \mathrm{Cl}_{2}: \mathrm{CD}_{3} \mathrm{CN}$ (5:1) showing either two separate signals for protons $9-\mathrm{H}, 9 \mathrm{u}-\mathrm{H}$ in case of slow rotation or a single signal in the fast rotor $\left[\mathrm{H}_{2}(\mathbf{1})\right]^{2+}$. 


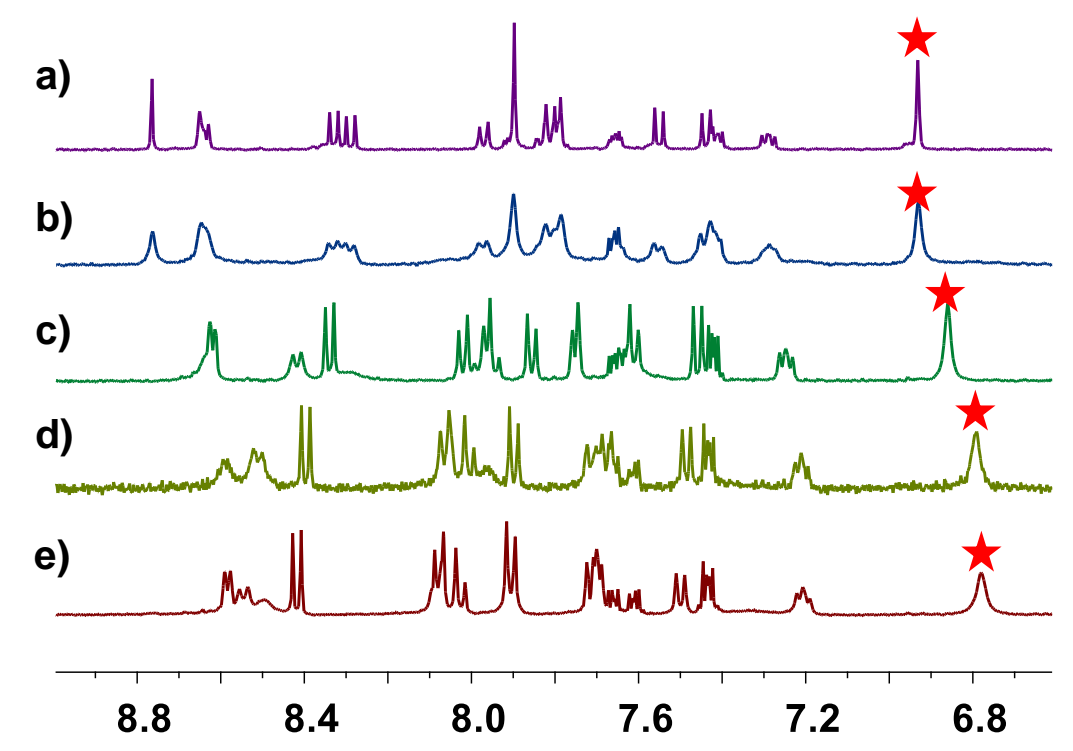

Figure S26. Partial ${ }^{1} \mathrm{H}$ NMR (400 MHz, $298 \mathrm{~K}$ ) in $\mathrm{CD}_{2} \mathrm{Cl}_{2}$ of (a) 1, (b) after addition of 0.5 equiv of TFA to 1 , (c) after addition of 0.5 equiv of more TFA to solution $b$, (d) after addition of 0.5 equiv of more TFA to solution c, (e) after addition of 0.5 equiv of more TFA to solution d.

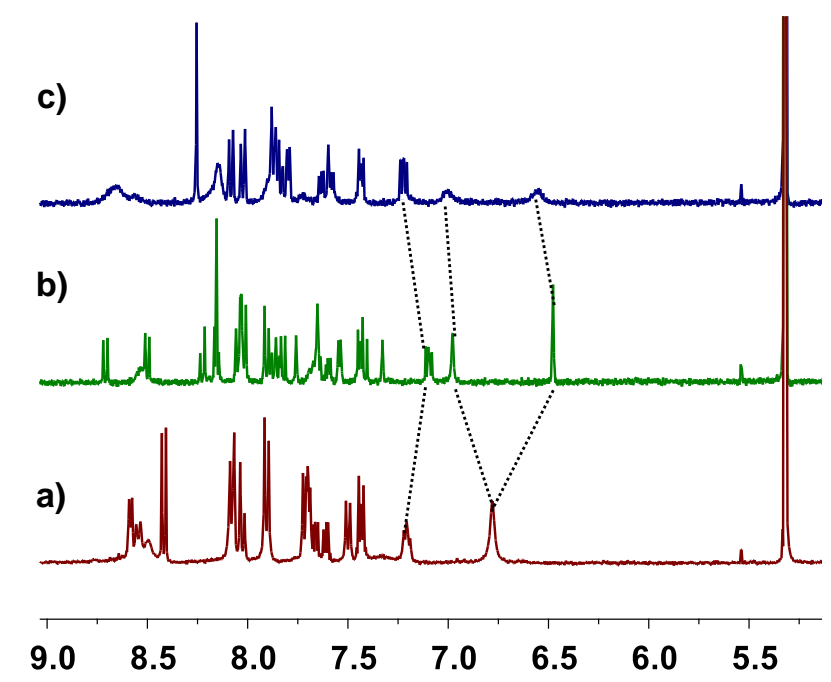

Figure S27. Partial ${ }^{1} \mathrm{H}$ NMR (400 MHz, $298 \mathrm{~K}$ ) in $\mathrm{CD}_{2} \mathrm{Cl}_{2}$ of (a) $\left[\mathrm{H}_{2}(\mathbf{1})\right]^{2+}$, (b) after addition of 2.0 equiv of $\mathrm{LiPF}_{6}$ as a standard solution in $\mathrm{CD}_{3} \mathrm{CN}$ to the solution a, (c) after addition of 2.0 equiv of $\mathrm{AgBF}_{4}$ as a standard solution in $\mathrm{CD}_{3} \mathrm{CN}$ to solution $\mathrm{b}$. 


\section{Fueling experiments}

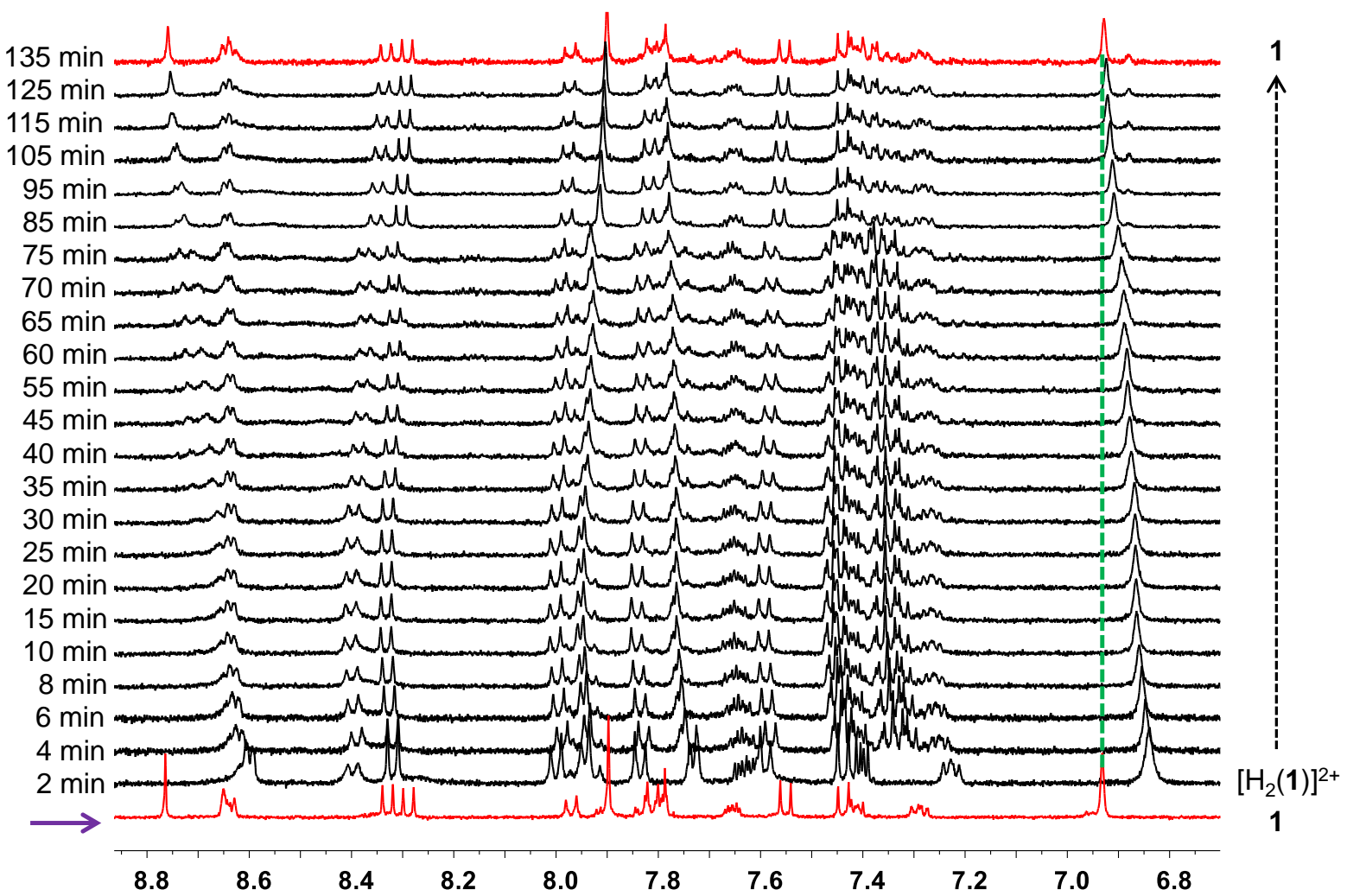

Figure S28. Partial ${ }^{1} \mathrm{H}$ NMR $(400 \mathrm{MHz}, 298 \mathrm{~K})$ of $\mathbf{1}(0.9 \mathrm{mM})$ in $\mathrm{CD}_{2} \mathrm{Cl}_{2}$ (bottom in red).

Changes in the ${ }^{1} \mathrm{H}$ NMR after addition of two equiv of acid $\mathbf{2}$ showing the formation of $\mathbf{1}$ from $\left[\mathrm{H}_{2}(\mathbf{1})\right]^{2+}$ with time.

B)

A)

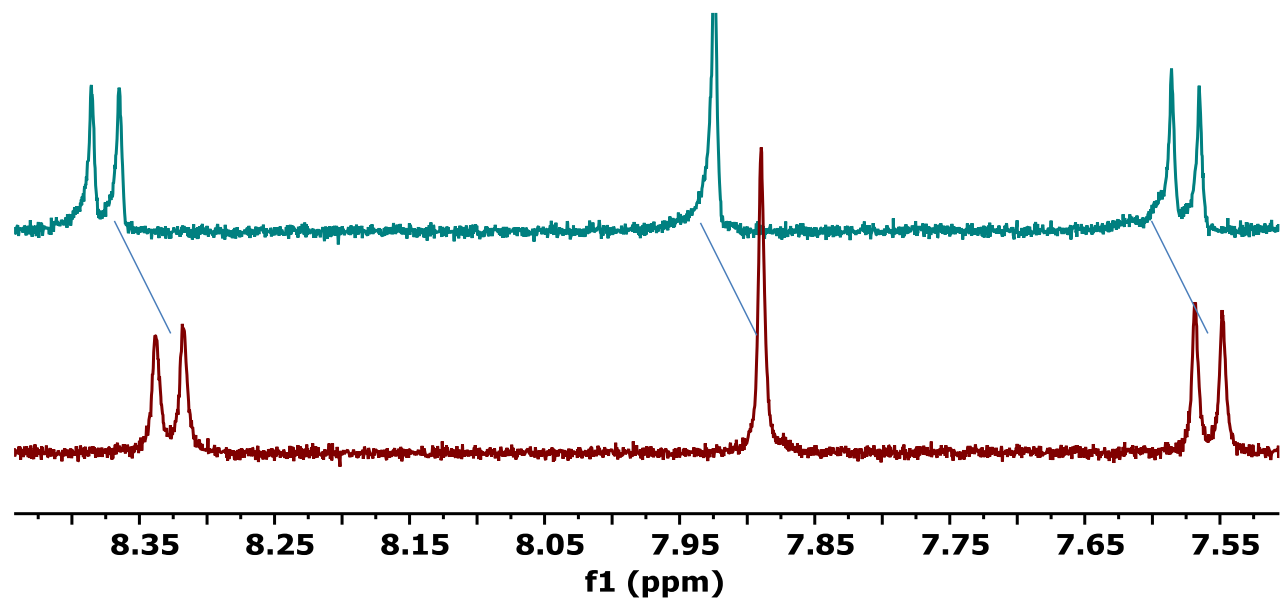

Figure S29. (A) Partial ${ }^{1} \mathrm{H}$ NMR (400 MHz, 298 K) of 2,9-dimesityl-1,10-phenanthroline,

(B) after addition of 1.0 equiv. of acid 2 to A. 


\section{General procedure of the silver(I) fueling}

Solid reactants were transferred to the NMR tube and dissolved in $\mathrm{CD}_{2} \mathrm{Cl}_{2}: \mathrm{CD}_{3} \mathrm{CN}$ (5:1), whereas liquids were taken as standard solutions. The ${ }^{1} \mathrm{H}$ NMR was measured with time and the yield of the substitution product 6 (singlet at $\delta 4.78 \mathrm{ppm}$ ) was determined using 1,1,2,2tetrachloroethane (17) as an internal standard (singlet at $\delta 6.05 \mathrm{ppm})$.
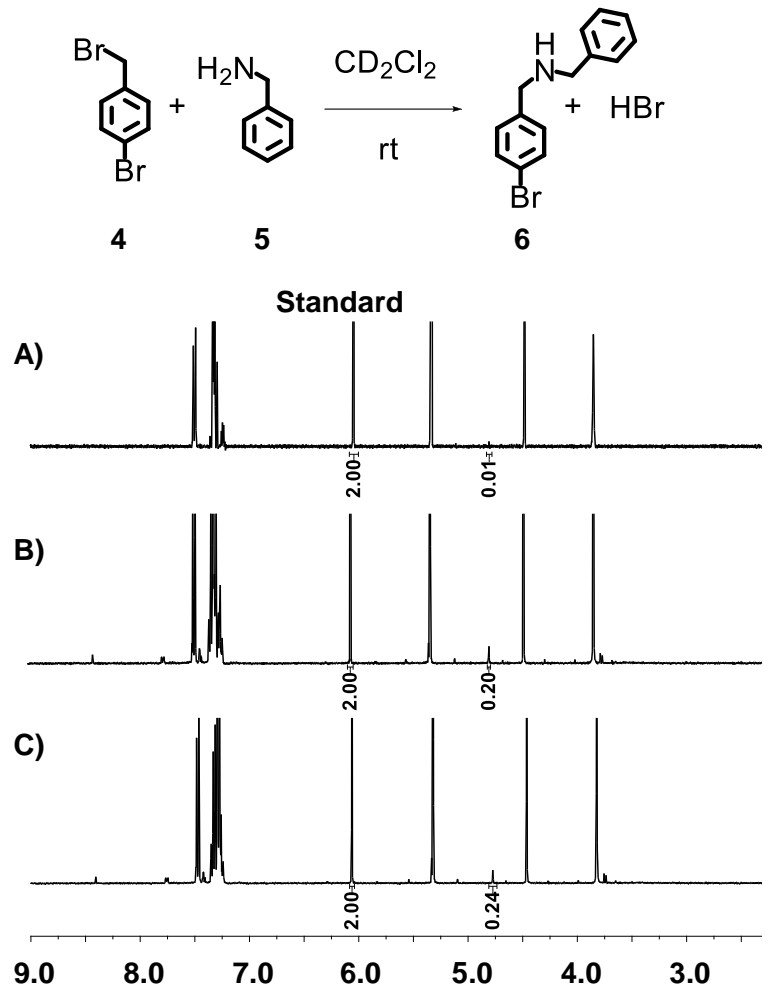

Figure S30. Partial ${ }^{1} \mathrm{H}$ NMR (400 MHz, $\mathrm{CD}_{2} \mathrm{Cl}_{2}: \mathrm{CD}_{3} \mathrm{CN}$ (5:1)). (A) After 20 min of mixing of 4, 5 and 1,1,2,2-tetrachloroethane $(17)\left(4.0 \times 10^{-3} \mathrm{M}\right)$ showing $<1 \%$ of 6 formation. (B) After 20 min of mixing of $\mathrm{AgBF}_{4}, \mathbf{4}, \mathbf{5}$ and $\mathbf{1 7}\left(4.0 \times 10^{-3} \mathrm{M}\right)$ in 1:10:10:10 ratio showing 10\% of product 6. (C) Mixture B after 160 min showing $12 \%$ of product 6. 


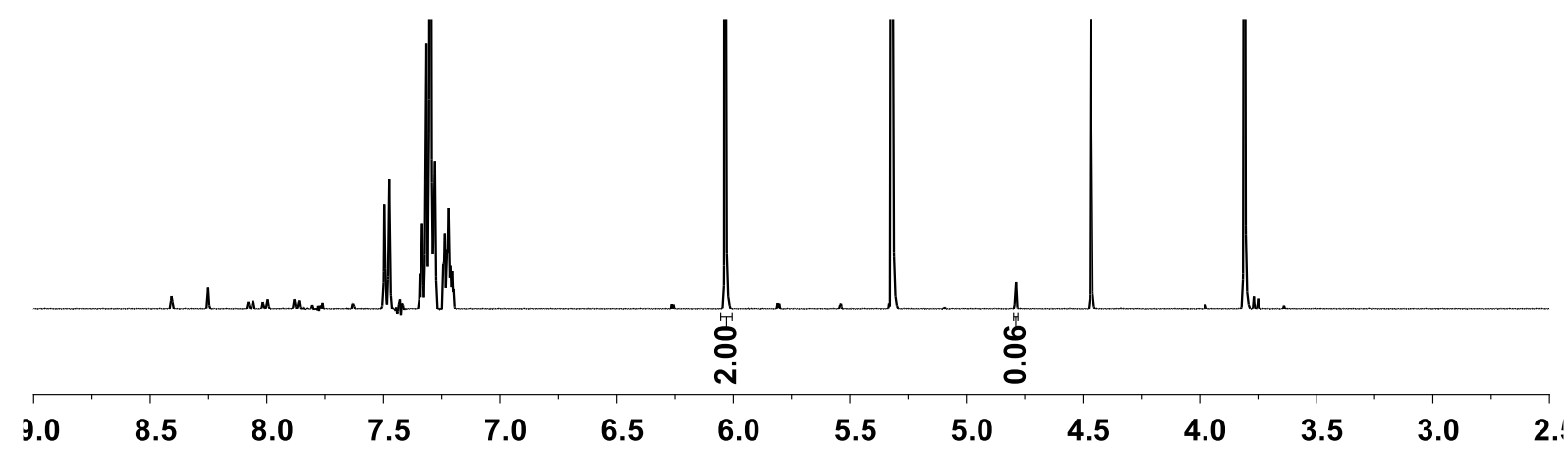

Figure S31. Partial ${ }^{1} \mathrm{H}$ NMR (400 MHz, $\left.\mathrm{CD}_{2} \mathrm{Cl}_{2}: \mathrm{CD}_{3} \mathrm{CN}(5: 1)\right)$ after 20 min of mixing of 1 $(125 \mu \mathrm{M}), \mathrm{AgBF}_{4}(250 \mu \mathrm{M}), \mathbf{4}(4.0 \mathrm{mM}), \mathbf{5}(4.0 \mathrm{mM})$ and $17(4.0 \mathrm{mM})$ showing formation of $3 \%$ of 6.
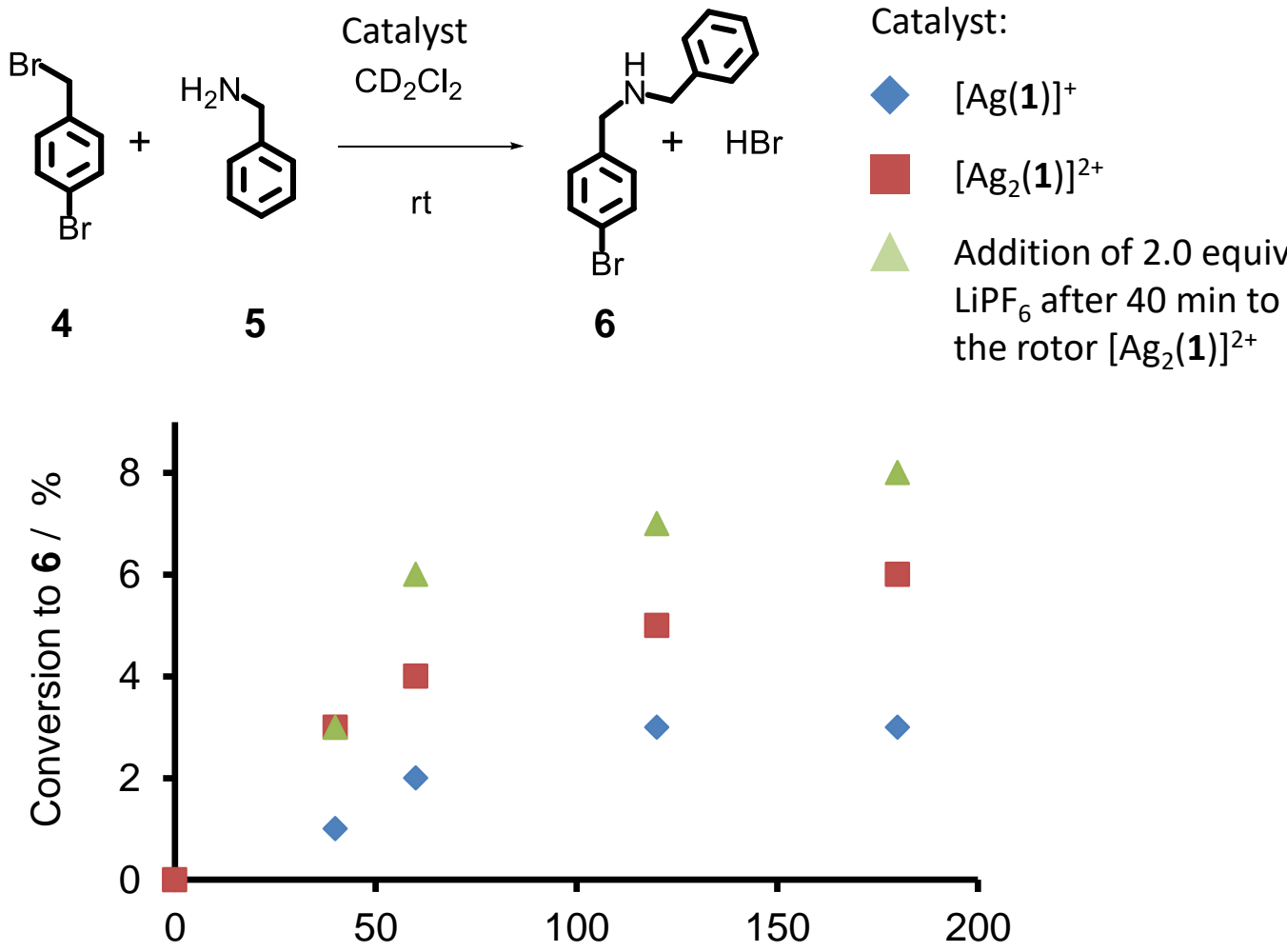

Time / $\min$

Figure S32. Reaction profile of the substitution reaction between $\mathbf{4}$ and $\mathbf{5}$ (both $4.0 \mathrm{mM}$ ) in presence of different silver(I) salts $\left(125 \mu \mathrm{M}\right.$ of $[\operatorname{Ag}(\mathbf{1})]^{+}$or $\left.\left[\operatorname{Ag}_{2}(\mathbf{1})\right]^{2+}\right)$. Addition of 2.0 equivalent of $\mathrm{LiPF}_{6}$ after $40 \mathrm{~min}$ to the rotor $\left[\mathrm{Ag}_{2}(\mathbf{1})\right]^{2+}$ increased the rate of the substitution. The reactions were monitored through ${ }^{1} \mathrm{H}$ NMR spectroscopy using $17(4.0 \mathrm{mM})$ as an internal standard. 

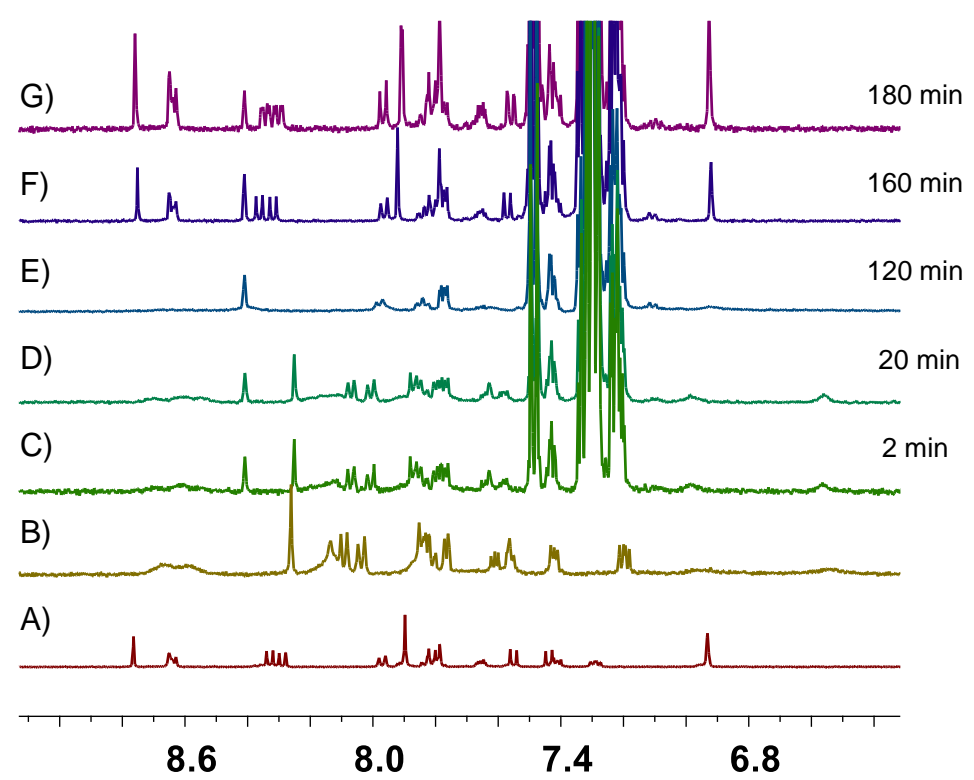

Figure S33. Partial ${ }^{1} \mathrm{H}$ NMR (400 MHz, $\left.\mathrm{CD}_{2} \mathrm{Cl}_{2}: \mathrm{CD}_{3} \mathrm{CN}(5: 1)\right)$ of (A) 1, (B) $\left[\mathrm{Ag}_{2}(\mathbf{1})\right]^{2+},(\mathrm{C})$ at $2 \mathrm{~min}$ after mixing of $\mathbf{1}(125 \mu \mathrm{M}), \mathrm{AgBF}_{4}(250 \mu \mathrm{M}), \mathbf{4}(4.0 \mathrm{mM}), \mathbf{5}(4.0 \mathrm{mM})$ and $17(4.0$ $\mathrm{mM}$ ) at room temperature, (D) after $20 \mathrm{~min},(\mathrm{E})$ after $120 \mathrm{~min},(\mathrm{~F})$ after $160 \mathrm{~min},(\mathrm{G})$ after 180 min.

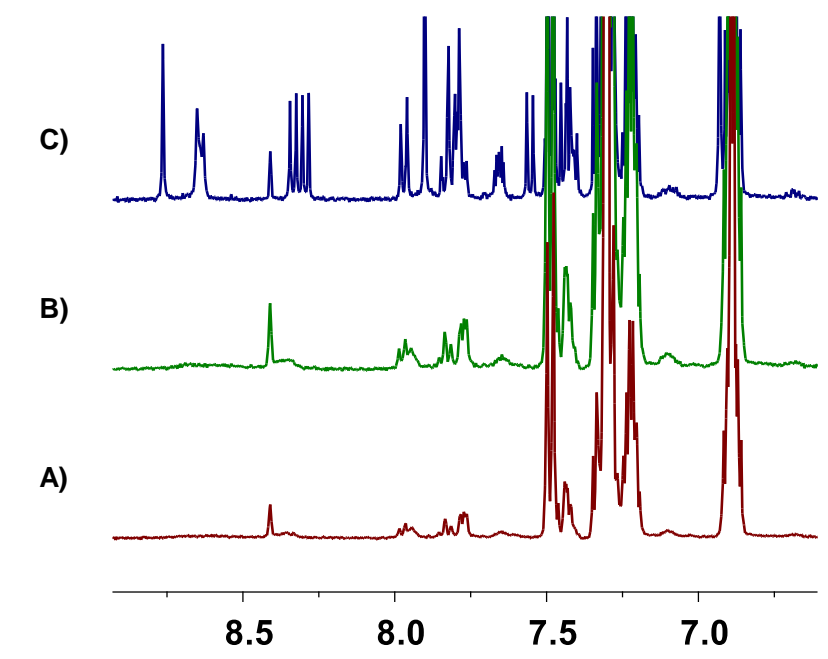

Figure S34. Partial ${ }^{1} \mathrm{H}$ NMR (400 MHz, $\left.\mathrm{CD}_{2} \mathrm{Cl}_{2}: \mathrm{CD}_{3} \mathrm{CN}(5: 1)\right)$ of the mixture of $1(125 \mu \mathrm{M})$, $\mathrm{AgBF}_{4}(250 \mu \mathrm{M}), \mathbf{4}(4.0 \mathrm{mM}), \mathbf{5}(4.0 \mathrm{mM}), 17(4.0 \mathrm{mM})$ and crown 7 (4.0 mM) (A) after 30 min, (B) after $60 \mathrm{~min},(\mathrm{C})$ after $90 \mathrm{~min}$. It shows the completion of $\mathrm{Ag}^{+}$fueling in $90 \mathrm{~min}$. 

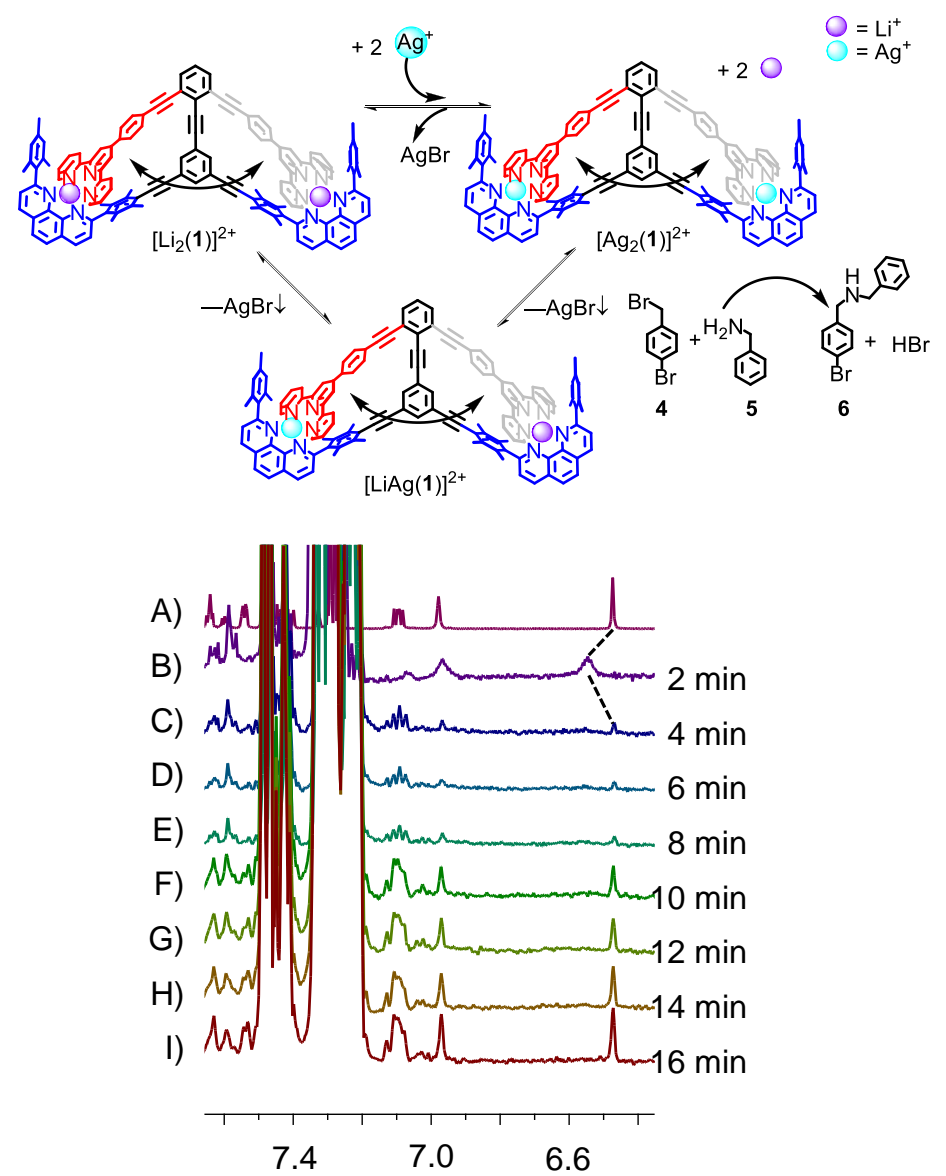

Figure S35. Partial ${ }^{1} \mathrm{H}$ NMR (400 MHz, $\mathrm{CD}_{2} \mathrm{Cl}_{2}: \mathrm{CD}_{3} \mathrm{CN}(5: 1)$ ) of a mixture of (A) 1 (125 $\mu \mathrm{M}), \mathrm{Li}^{+}(250 \mu \mathrm{M}), \mathbf{4}(4.0 \mathrm{mM}), \mathbf{5}(4.0 \mathrm{mM})$ and $17(4.0 \mathrm{mM})$. (B-I) after addition of 2.0 equiv of $\mathrm{AgBF}_{4}$ (with respect to 1) to the mixture $\mathrm{A}$. The spectra show that no complex $\left[\operatorname{Ag}_{2}(\mathbf{1})\right]^{2+}$ is left after $16 \mathrm{~min}$ and rotor $\left[\operatorname{Li}_{2}(\mathbf{1})\right]^{2+}$ is formed.

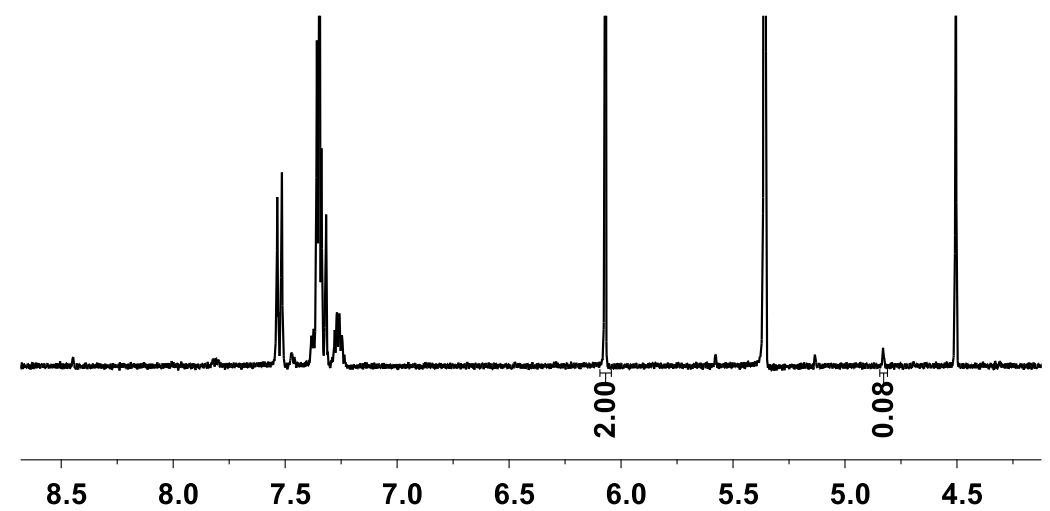

Figure S36. Partial ${ }^{1} \mathrm{H}$ NMR (400 MHz, $\mathrm{CD}_{2} \mathrm{Cl}_{2}: \mathrm{CD}_{3} \mathrm{CN}(5: 1)$ ) after 20 min of mixing of $\mathrm{LiPF}_{6}, \mathbf{4}, 5(4.0 \mathrm{mM})$ and $17(4.0 \mathrm{mM})$ in 1:10:10:10 ratio, showing the formation of $4 \%$ of 6 after $20 \mathrm{~min}$. 


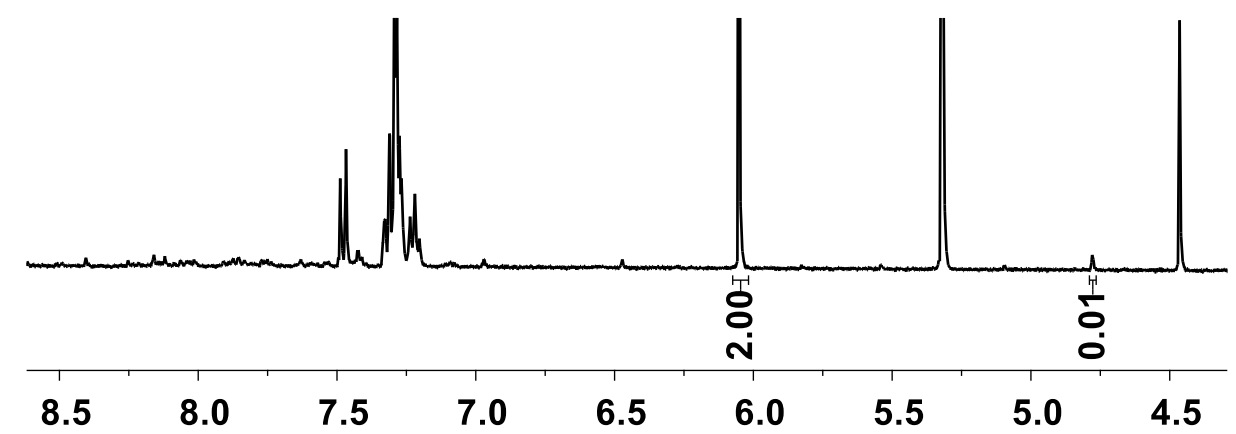

Figure S37. Partial ${ }^{1} \mathrm{H}$ NMR (400 MHz, $\left.\mathrm{CD}_{2} \mathrm{Cl}_{2}: \mathrm{CD}_{3} \mathrm{CN}(5: 1)\right)$ after 20 min of mixing of $\left[\operatorname{Li}_{2}(\mathbf{1})\right]^{2+}, \mathbf{4}, \mathbf{5}(4.0 \mathrm{mM})$ and $\mathbf{1 7}(4.0 \mathrm{mM})$ in 1:10:10:10 ratio, showing the formation of $<1 \%$ of 6 after 20 min.
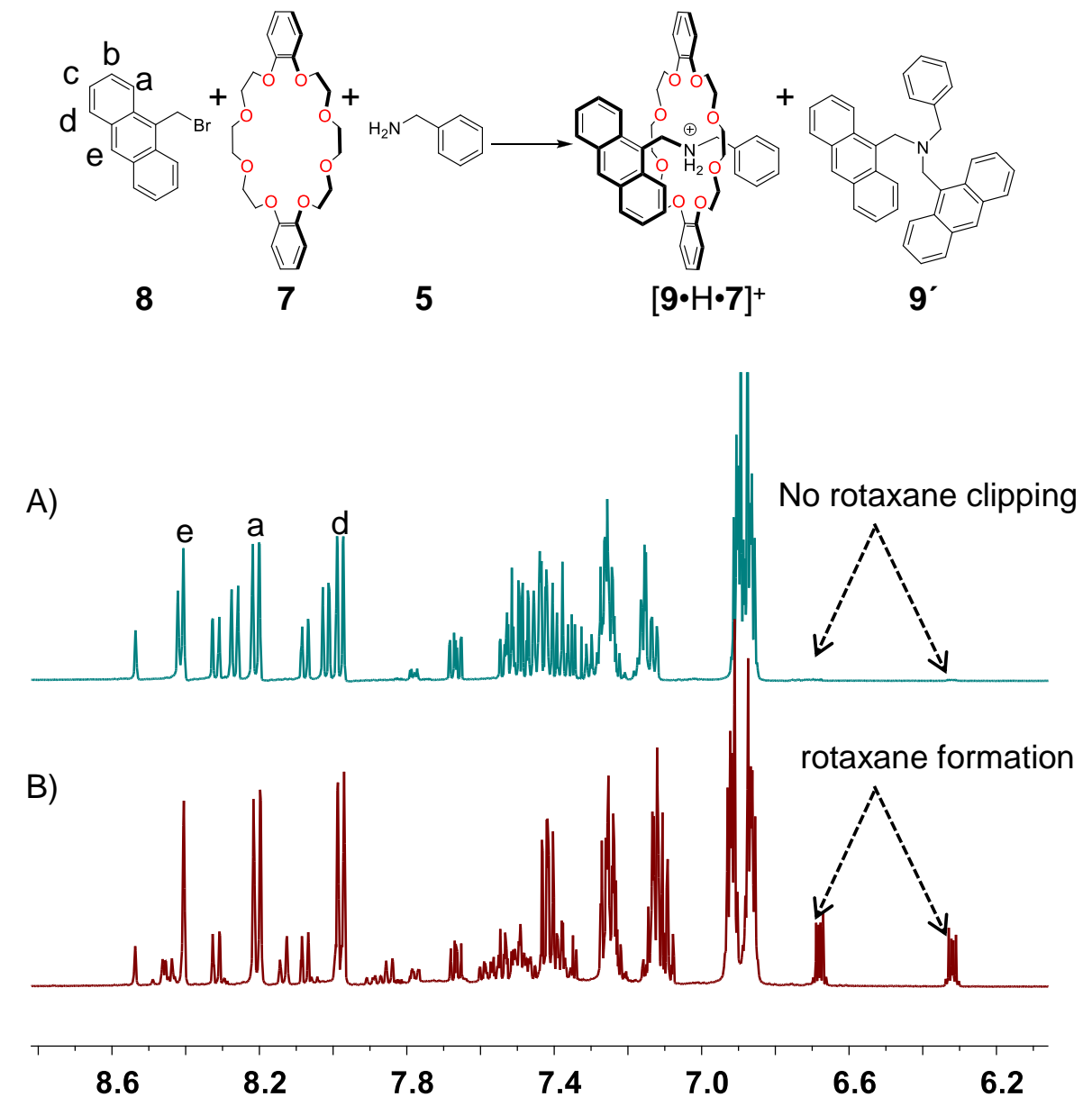

Figure S38. Partial ${ }^{1} \mathrm{H}$ NMR (400 MHz, $\left.\mathrm{CD}_{2} \mathrm{Cl}_{2}\right)$ of a mixture of (A) 5 (4.0 mM), 7 and 8 in 1:1:1 ratio after heating at $50{ }^{\circ} \mathrm{C}$ for $2 \mathrm{~h}$. No rotaxane clipping is yet detectable but significant 
amounts of $\mathrm{HBr}$ as a side product $(\sim 50 \%)$, indicating that $\mathrm{HBr}$ is not a strong enough acid for in situ rotaxane clipping. (B) 15 min after addition of $\mathrm{AgBF}_{4}$ (as solid) to the mixture of 5 (4.0 mM), 7 and $\mathbf{8}$ in 1:5:5:5 ratio at room temperature, it shows ( 9\%) rotaxane clipping, ${ }^{5}$ i.e. formation of $[\mathbf{9} \cdot \mathrm{H} \cdot \mathbf{7}]^{+}$, due to the presence of the strong acid $\mathrm{HBF}_{4}$. The yield was calculated considering 7 as standard.

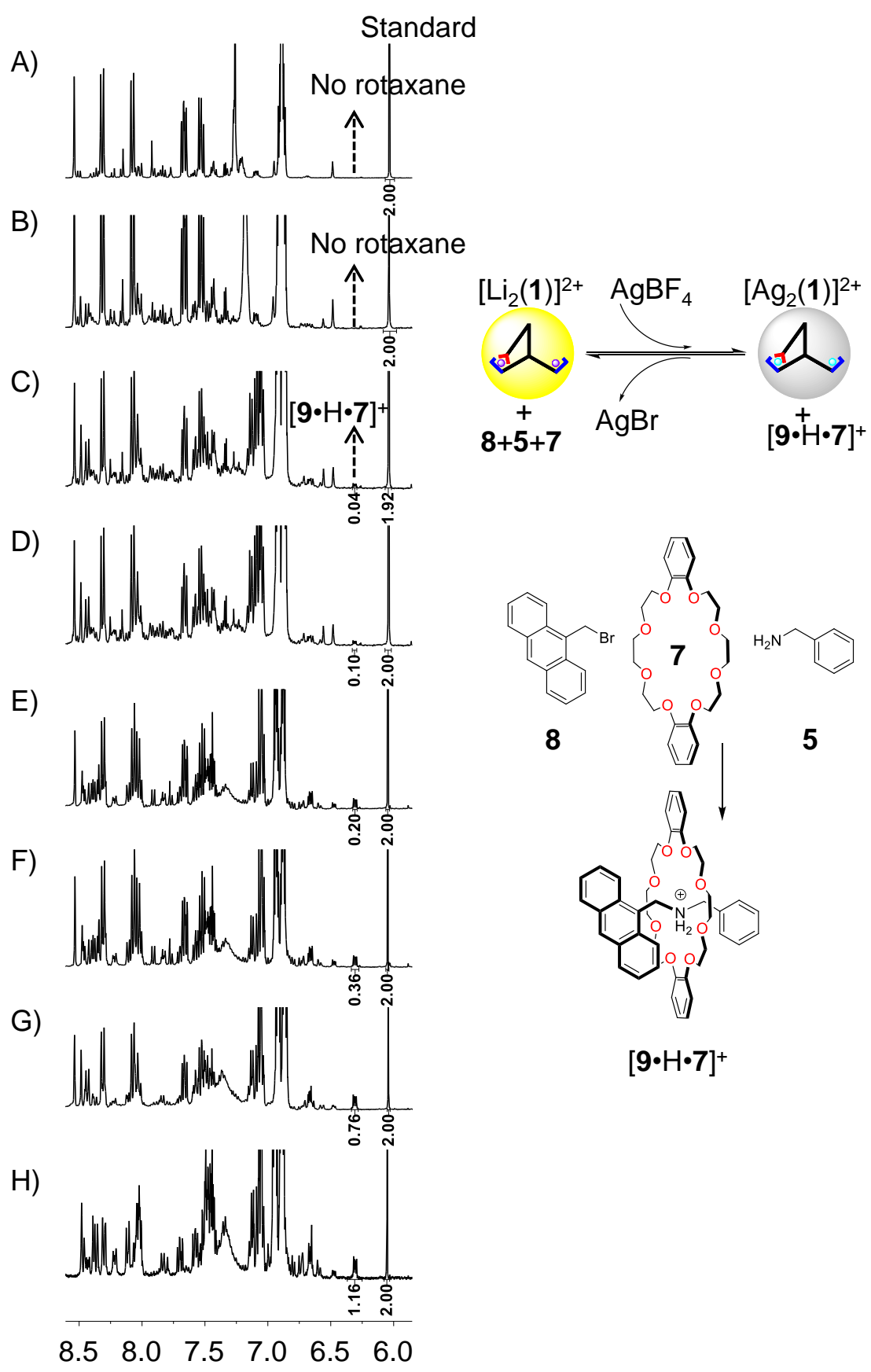

Figure S39. Partial ${ }^{1} \mathrm{H}$ NMR (400 MHz, $\left.\mathrm{CD}_{2} \mathrm{Cl}_{2}: \mathrm{CD}_{3} \mathrm{CN}(5: 1)\right)$ of a mixture of (A) 1 (125 $\mu \mathrm{M}), \mathrm{Li}^{+}(250 \mu \mathrm{M}), 5(4.0 \mathrm{mM})$ and $7(4.0 \mathrm{mM}), 8(4.0 \mathrm{mM})$ and $17(4.0 \mathrm{mM})$; (B) $15 \mathrm{~min}$ 
after the addition of 2.0 equiv of $\mathrm{AgBF}_{4}$ (with respect to 1) to the mixture $\mathrm{A}$, it shows no rotaxane formation ( $1^{\text {st }}$ cycle); (C) 15 min after the addition of 2.0 equiv of $\mathrm{AgBF}_{4}$ (with respect to 1) to the mixture $\mathrm{B}$, only $1 \%$ of rotaxane $[9 \cdot \mathrm{H} \cdot 7]^{+}$was furnished at the end of the $2^{\text {nd }}$ cycle; (D) 15 min after another addition of 2.0 equiv of $\mathrm{AgBF}_{4}$ (with respect to $\mathbf{1}$ ) to that mixture, $2.5 \%$ of rotaxane $[9 \cdot \mathrm{H} \cdot \mathbf{7}]^{+}$was furnished at the end of the $3^{\text {rd }}$ cycle; (E) 20 min after another addition of 2.0 equiv of $\mathrm{AgBF}_{4}$ (with respect to 1 ) to that mixture, $5 \%$ of rotaxane $[9 \cdot \mathrm{H} \cdot 7]^{+}$was furnished at the end of the $4^{\text {th }}$ cycle; (F) 20 min after another addition of 2.0 equiv of $\mathrm{AgBF}_{4}$ (with respect to $\mathbf{1}$ ) to that mixture, $9 \%$ of rotaxane $[\mathbf{9} \cdot \mathrm{H} \cdot \mathbf{7}]^{+}$was furnished at the end of the $5^{\text {th }}$ cycle; $(\mathrm{G}) 25 \mathrm{~min}$ after another addition of 2.0 equiv of $\mathrm{AgBF}_{4}$ (with respect to 1 ) to that mixture, $19 \%$ of rotaxane $[9 \cdot \mathrm{H} \cdot \mathbf{7}]^{+}$was furnished at the end of the $6^{\text {th }}$ cycle; $(\mathrm{H})$ 30 min after another addition of 2.0 equiv of $\mathrm{AgBF}_{4}$ (with respect to 1) to that mixture, 29\% of rotaxane $[\mathbf{9} \cdot \mathrm{H} \cdot \mathbf{7}]^{+}$was furnished at the end of the $7^{\text {th }}$ cycle. 


\section{Variable temperature studies, ROESY and determination of kinetic parameters}

The rotational rate constants of the proton rotor $\left[\mathrm{H}_{2}(\mathbf{1})\right]^{2+}$ at various temperatures was analyzed using the software WinDNMR ${ }^{6}$ through simulation of the experimental ${ }^{1} \mathrm{H}$ NMR spectra using the model of a 2-spin system undergoing mutual exchange. Activation parameters were determined from an Eyring plot.
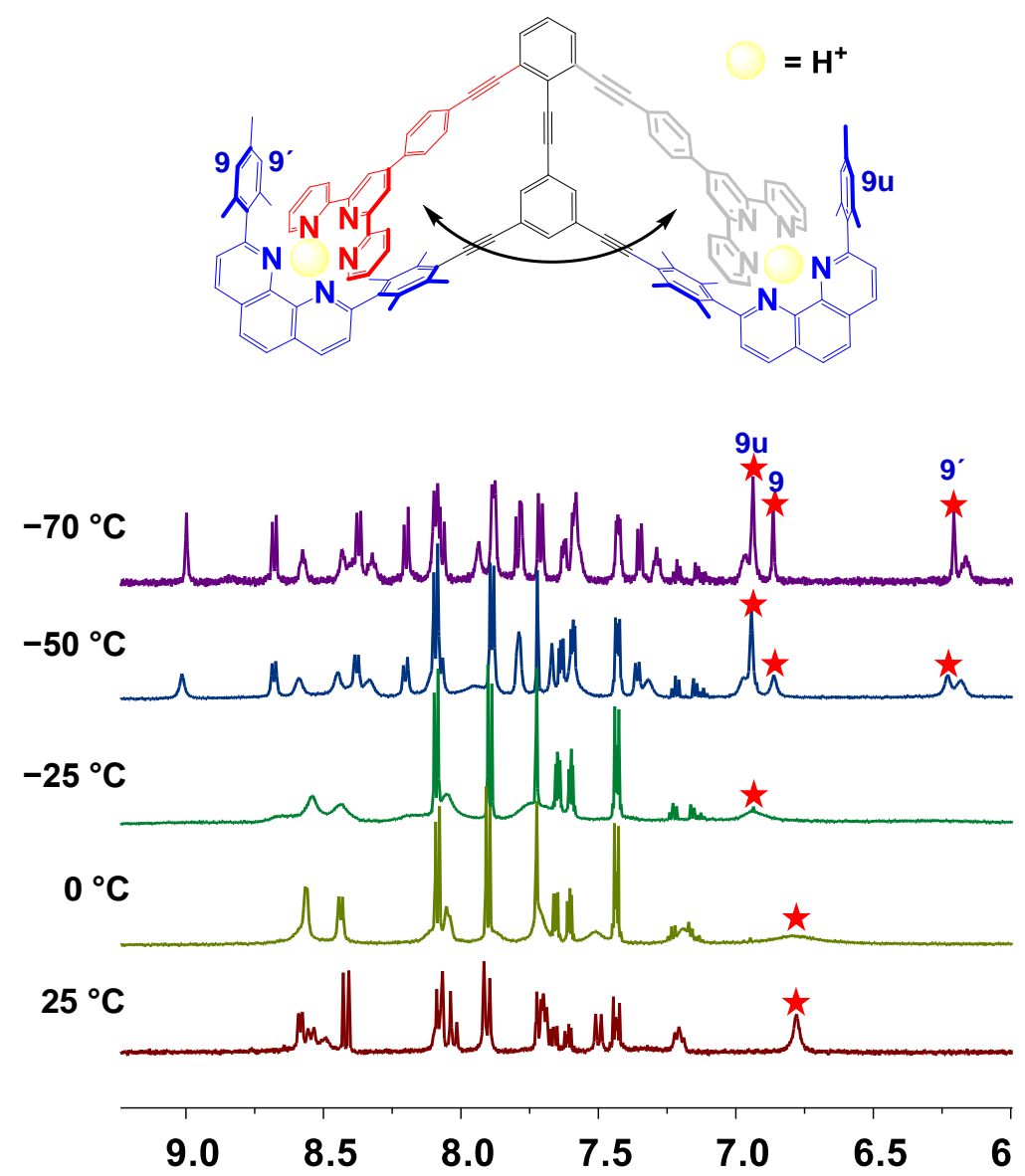

Figure S40. Partial ${ }^{1} \mathrm{H}$ VT-NMR spectra $\left(\mathrm{CD}_{2} \mathrm{Cl}_{2}, 600 \mathrm{MHz}\right)$ of $\left[\mathrm{H}_{2}(\mathbf{1})\right]^{2+}$ at different temperatures. At room temperature $\left(25^{\circ} \mathrm{C}\right)$, the mesityl proton $9-\mathrm{H}$ shows an averaged peak at $6.78 \mathrm{ppm}$ but is separated into three sets in 2:1:1 ratio at $6.20 \mathrm{ppm}\left(9^{\prime}-\mathrm{H}\right), 6.86 \mathrm{ppm}(9-\mathrm{H})$, $6.95 \mathrm{ppm}(9 \mathrm{u}-\mathrm{H})$ at $-70{ }^{\circ} \mathrm{C}$. 


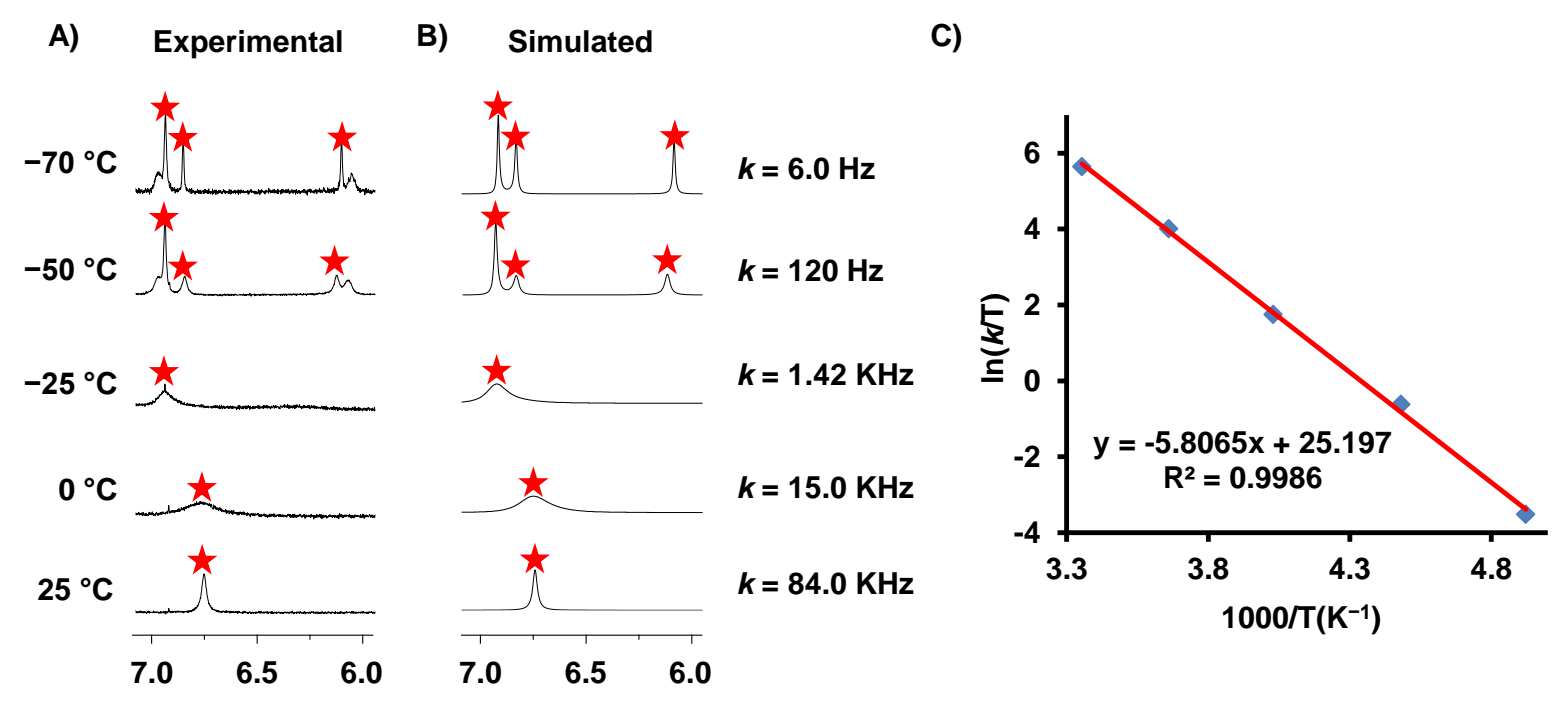

Figure S41. Partial ${ }^{1} \mathrm{H}$ VT-NMR $\left(\mathrm{CD}_{2} \mathrm{Cl}_{2}, 600 \mathrm{MHz}\right)$ of $\left[\mathrm{H}_{2}(\mathbf{1})\right]^{2+}$ showing (A) experimental and (B) theoretical splitting of proton 9-H at various temperatures. Simulation provided the corresponding rate constants. (C) Eyring plot for the rotational dynamics in $\left[\mathrm{H}_{2}(\mathbf{1})\right]^{2+}$, the kinetic parameters were determined as $\Delta H^{\ddagger}=48.3 \pm 0.6 \mathrm{~kJ} \mathrm{~mol}^{-1}, \Delta S^{\ddagger}=11.9 \pm 1.4 \mathrm{~J} \mathrm{~mol}^{-1} \mathrm{~K}^{-1}$ and $\Delta G^{\ddagger} 298=44.7 \pm 0.2 \mathrm{~kJ} \mathrm{~mol}^{-1}$.

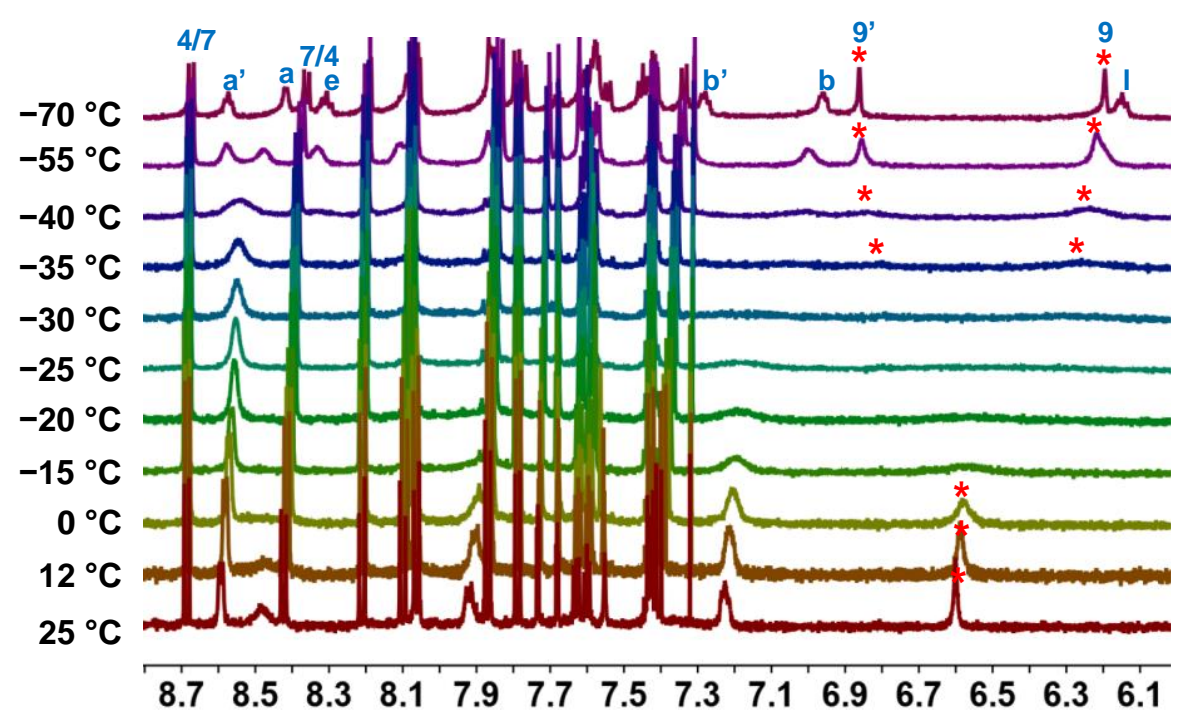

Figure S42. Partial VT ${ }^{1} \mathrm{H}-\mathrm{NMR}$ spectra $\left(\mathrm{CD}_{2} \mathrm{Cl}_{2}, 600 \mathrm{MHz}\right)$ of $\left[\mathrm{H}\left(\mathbf{1}^{\prime}\right)\right]^{+}$. Due to the $\pi$-cloud of the terpyridine, the triplet for $1-\mathrm{H}$ of $\left[\mathrm{H}\left(\mathbf{1}^{\prime}\right)\right]^{+}$is drastically shifted upfield $(6.14 \mathrm{ppm})$ at $-70{ }^{\circ} \mathrm{C}$. Notably, terpyridine protons $(\mathrm{a}-\mathrm{H}$ and $\mathrm{b}-\mathrm{H})$ are also split in 1:1 ratio, suggesting restricted rotation within the terpyridine unit. 
$\begin{array}{ll}\text { A) Experimental } & \text { B) Simulated }\end{array}$

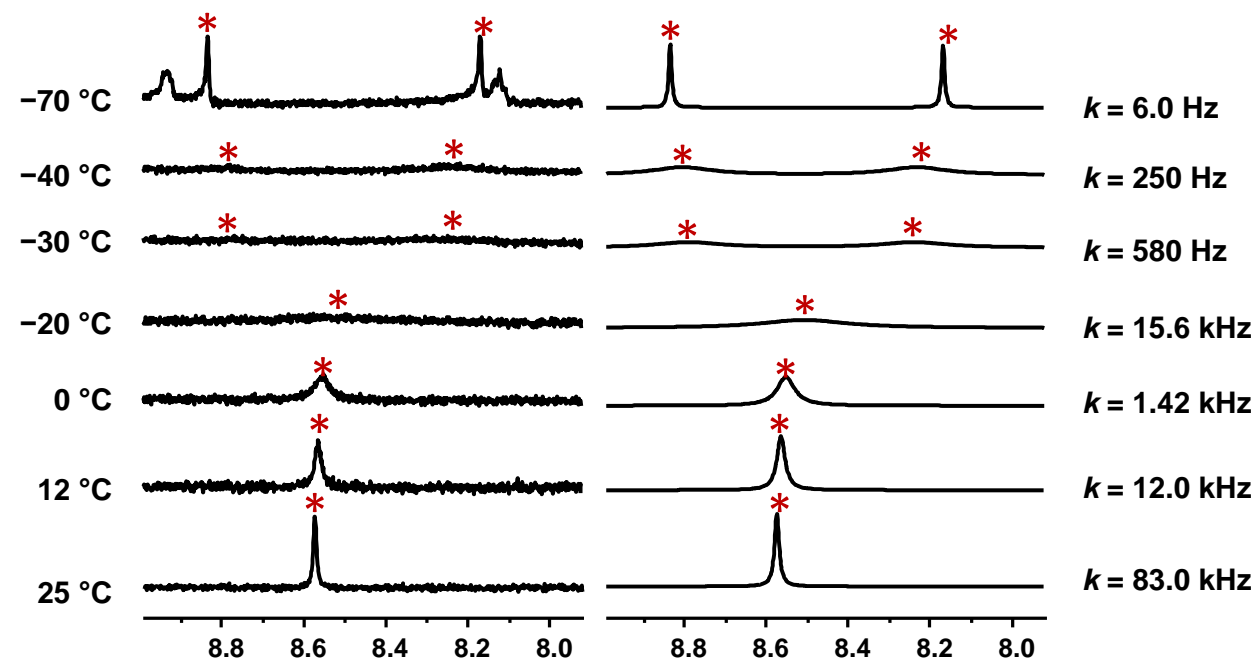

C)

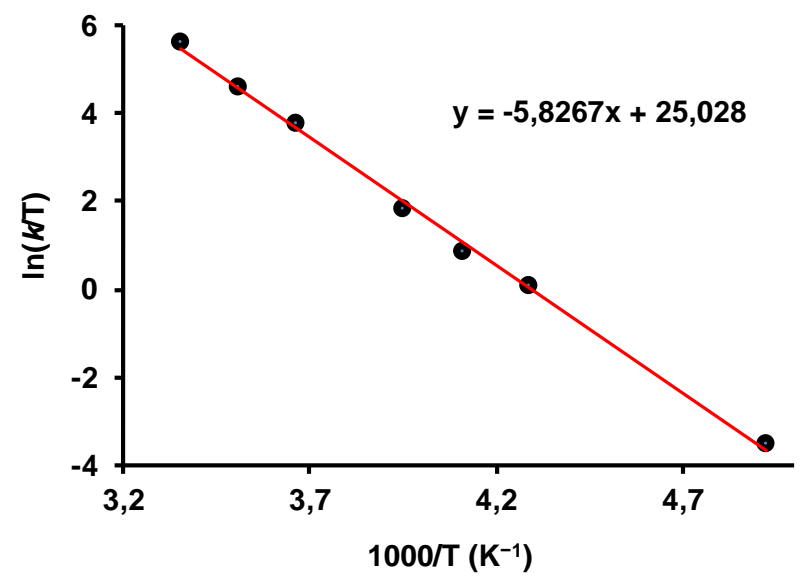

Figure S43. Partial ${ }^{1} \mathrm{H}$ VT-NMR $\left(\mathrm{CD}_{2} \mathrm{Cl}_{2}, 600 \mathrm{MHz}\right)$ of $\left[\mathrm{H}\left(\mathbf{1}^{\text {'}}\right)\right]^{+}$showing (A) experimental and (B) simulated splitting of proton $9-\mathrm{H}$ at different temperatures. Simulation provided the corresponding rate constants. (C) Eyring plot for the rotational dynamics in $\left[\mathrm{H}\left(\mathbf{1}^{\prime}\right)\right]^{+}$: The activation data were determined as $\Delta H^{\ddagger}=48.4 \pm 0.7 \mathrm{~kJ} \mathrm{~mol}^{-1}, \Delta S^{\ddagger}=10.5 \pm 2.1 \mathrm{~J} \mathrm{~mol}^{-1} \mathrm{~K}^{-1}$ and $\Delta G^{\ddagger} 298=45.3 \pm 0.6 \mathrm{~kJ} \mathrm{~mol}^{-1}$. 


\section{Rate constant calculation by volume intensity of the ROESY cross peaks}

The slow exchange of metal rotors was determined by ${ }^{1} \mathrm{H}-{ }^{1} \mathrm{H}$ ROESY NMR (mixing time $=$ $0.3 \mathrm{~s}$ ) experiment with volume intensity of cross and diagonal peaks. The rotational exchange frequency was calculated using the equation: $I_{\mathrm{D}} / I_{\mathrm{C}}=\left(1-k \mathrm{t}_{\mathrm{M}}\right) / k \mathrm{t}_{\mathrm{M}} \quad$ or $\quad k=1 /\left[\mathrm{t}_{\mathrm{M}}\left(I_{\mathrm{D}} / I_{\mathrm{C}}+1\right)\right]$

$I_{\mathrm{D}}$ : intensity of diagonal peak

$I_{C}$ : intensity of cross peak

$\mathrm{t}_{\mathrm{M}}$ : mixing time $(0.3 \mathrm{~s})$

Free energy of activation was calculated using the eyring equation;

$k=\left(\mathrm{k}_{\mathrm{B}} \mathrm{T} / \mathrm{h}\right) \exp \left(-\Delta G^{\neq} / \mathrm{RT}\right)$

$\mathrm{k}_{\mathrm{B}}$ : Boltzmann constant

h: Planck's constant

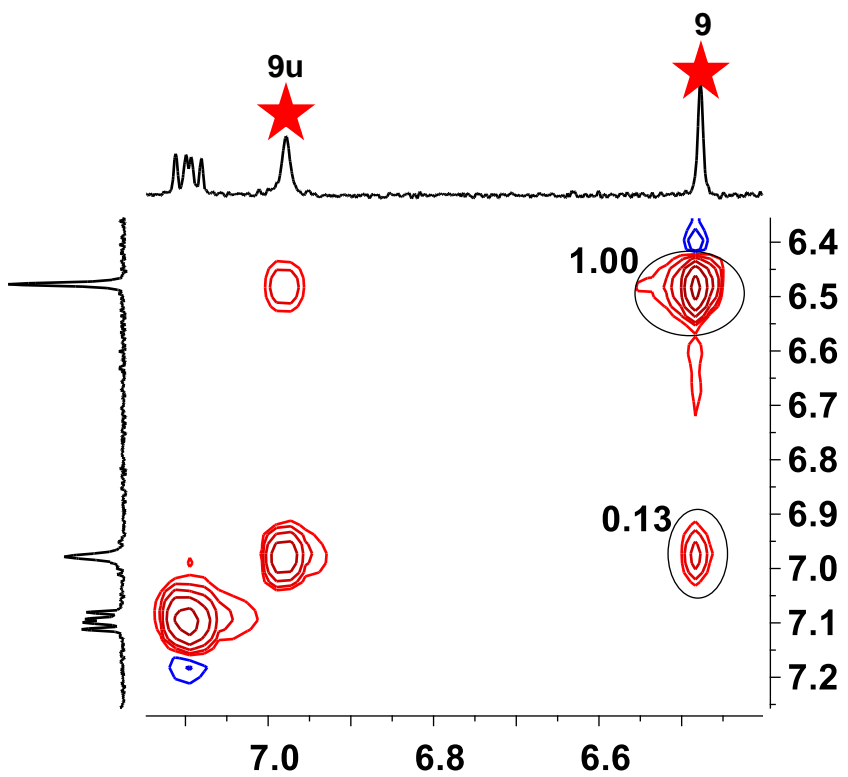

Figure S44. Partial ${ }^{1} \mathrm{H}-{ }^{1} \mathrm{H}$ ROESY $\left(400 \mathrm{MHz}, \mathrm{CD}_{2} \mathrm{Cl}_{2}: \mathrm{CD}_{3} \mathrm{CN}(5: 1)\right)$ of $\left[\mathrm{Li}_{2}(\mathbf{1})\right]^{2+}$ with volume intensity of cross and diagonal peaks. The rotational frequency is determined as $k_{298}=$ $0.38 \mathrm{~Hz}$ and the free energy of activation $\Delta G^{\ddagger} 298=75.7 \mathrm{~kJ} \mathrm{~mol}^{-1}$. 


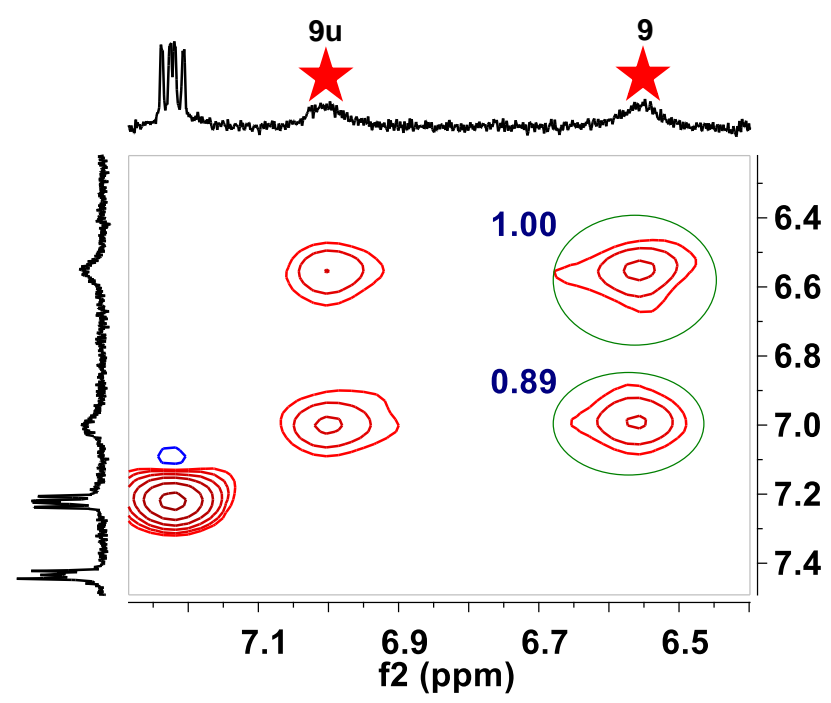

Figure S45. Partial ${ }^{1} \mathrm{H}^{-1} \mathrm{H}$ ROESY $\left(400 \mathrm{MHz}, \mathrm{CD}_{2} \mathrm{Cl}_{2}: \mathrm{CD}_{3} \mathrm{CN}(5: 1)\right)$ of $\left[\mathrm{Ag}_{2}(\mathbf{1})\right]^{2+}$ with volume intensity of cross and diagonal peaks. The rotational frequency is determined as $k_{298}$ $=1.57 \mathrm{~Hz}$ and the free energy of activation $\Delta G^{\ddagger} 298=72.2 \mathrm{~kJ} \mathrm{~mol}^{-1}$.

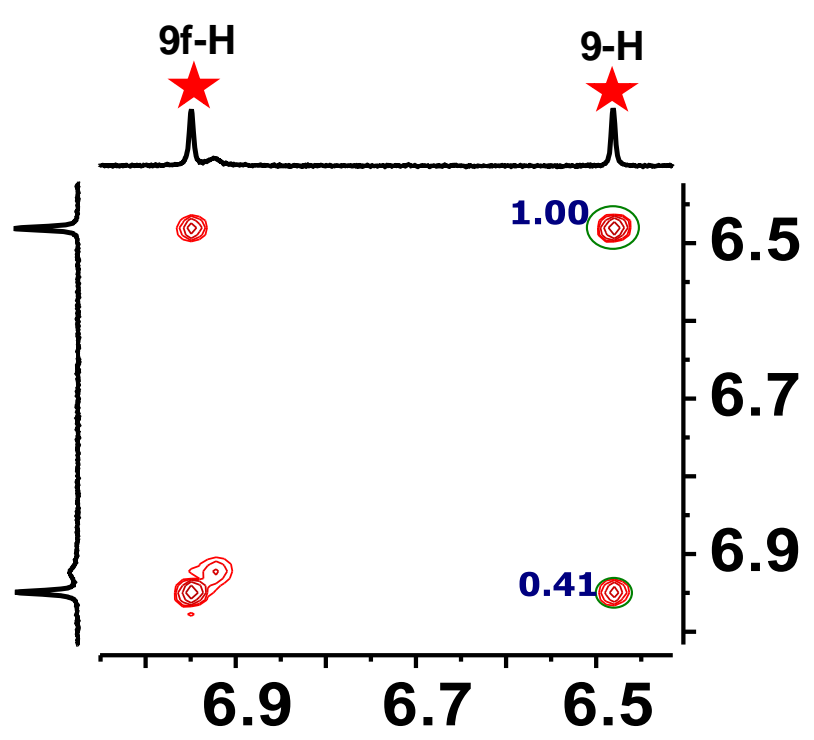

Figure S46. Partial ${ }^{1} \mathrm{H}^{-}{ }^{1} \mathrm{H}$ ROESY $\left(600 \mathrm{MHz}, \mathrm{CD}_{2} \mathrm{Cl}_{2}\right.$ : $\left.\mathrm{CD}_{3} \mathrm{CN}(5: 1)\right)$ of $[\mathrm{Li}(\mathbf{1})]^{+}$showing volume intensity of cross and diagonal peaks. The rotational frequency was determined as $k_{298}=0.97 \mathrm{~Hz}$ and the free energy of activation $\Delta G^{\neq}{ }_{298}=73.1 \mathrm{~kJ} \mathrm{~mol}^{-1}$. Rotation in $[\mathrm{Li}(\mathbf{1})]^{+}$ requires that the terpyridine head travels together with the lithium ion to the second phenanthroline site. 


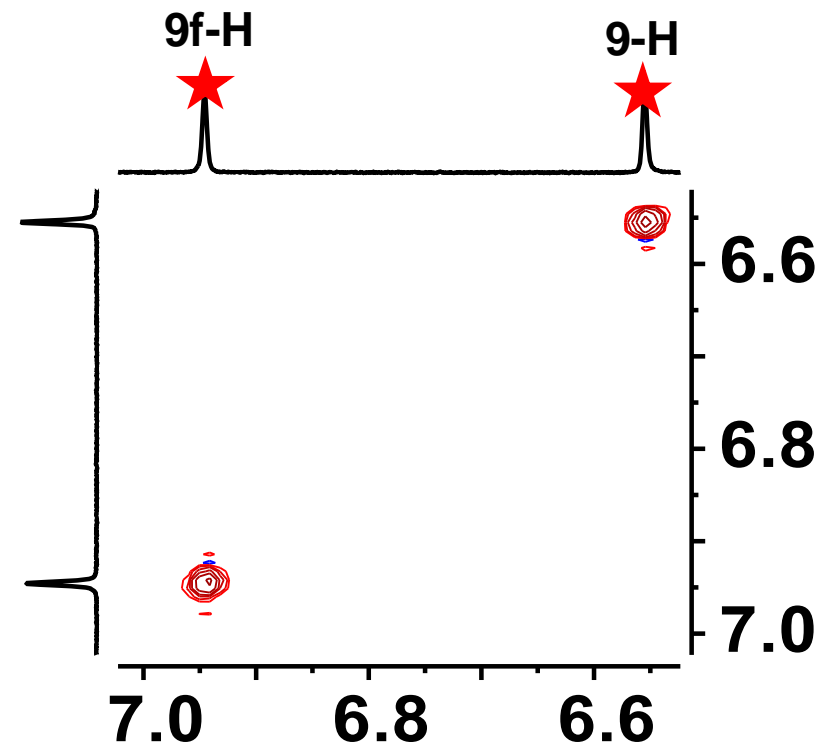

Figure S47. Partial ${ }^{1} \mathrm{H}-{ }^{1} \mathrm{H}$ ROESY $\left(600 \mathrm{MHz}, \mathrm{CD}_{2} \mathrm{Cl}_{2}\right.$ : $\left.\mathrm{CD}_{3} \mathrm{CN}(5: 1)\right)$ of $[\mathrm{Ag}(\mathbf{1})]^{+}$with volume intensity of diagonal peaks. No cross peaks were found. 


\section{ESI-MS spectra}

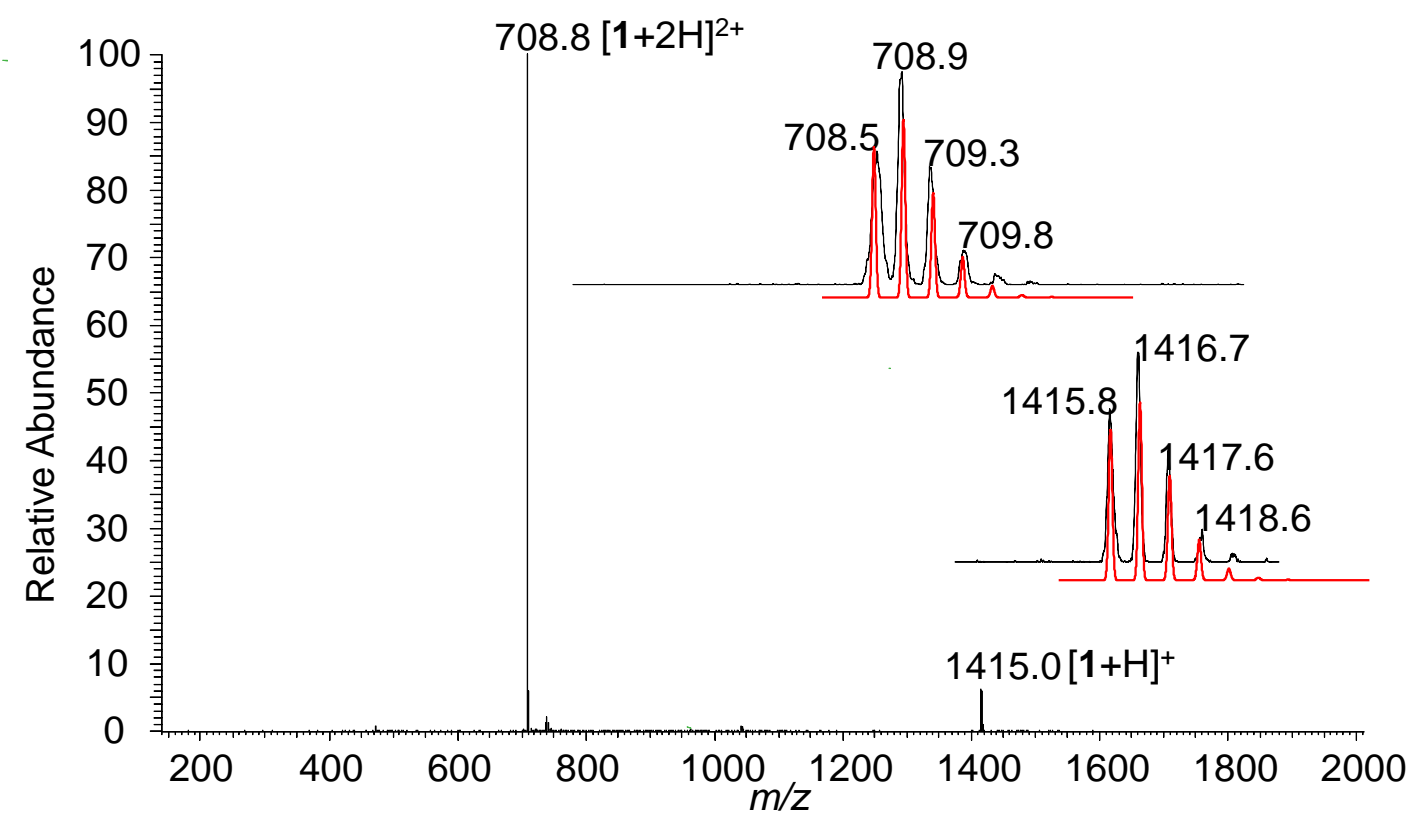

Figure S48. ESI-MS of compound 1 after protonation.

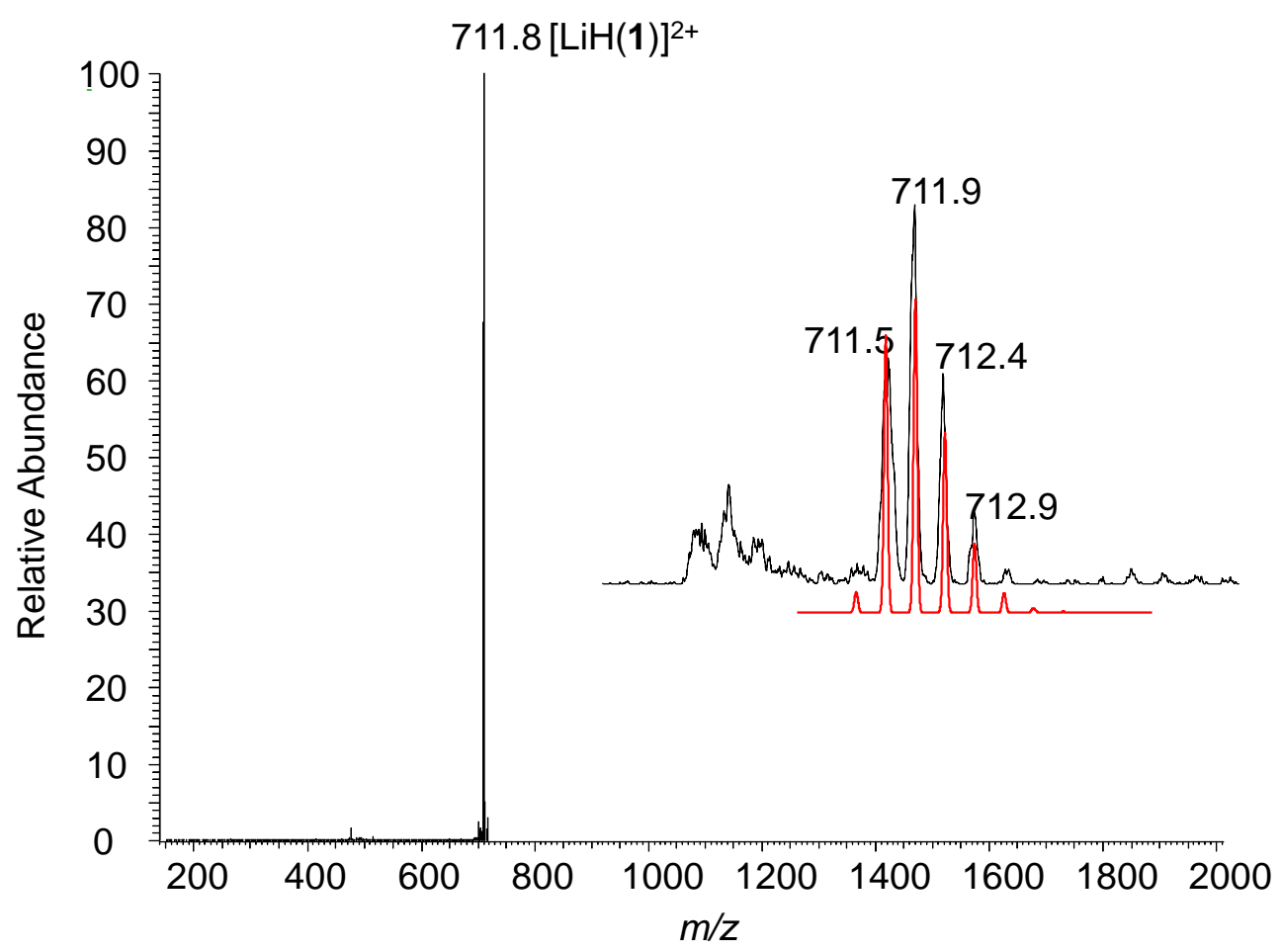

Figure S49. ESI-MS of $\left[\operatorname{Li}_{2}(\mathbf{1})\right]^{2+}$. 


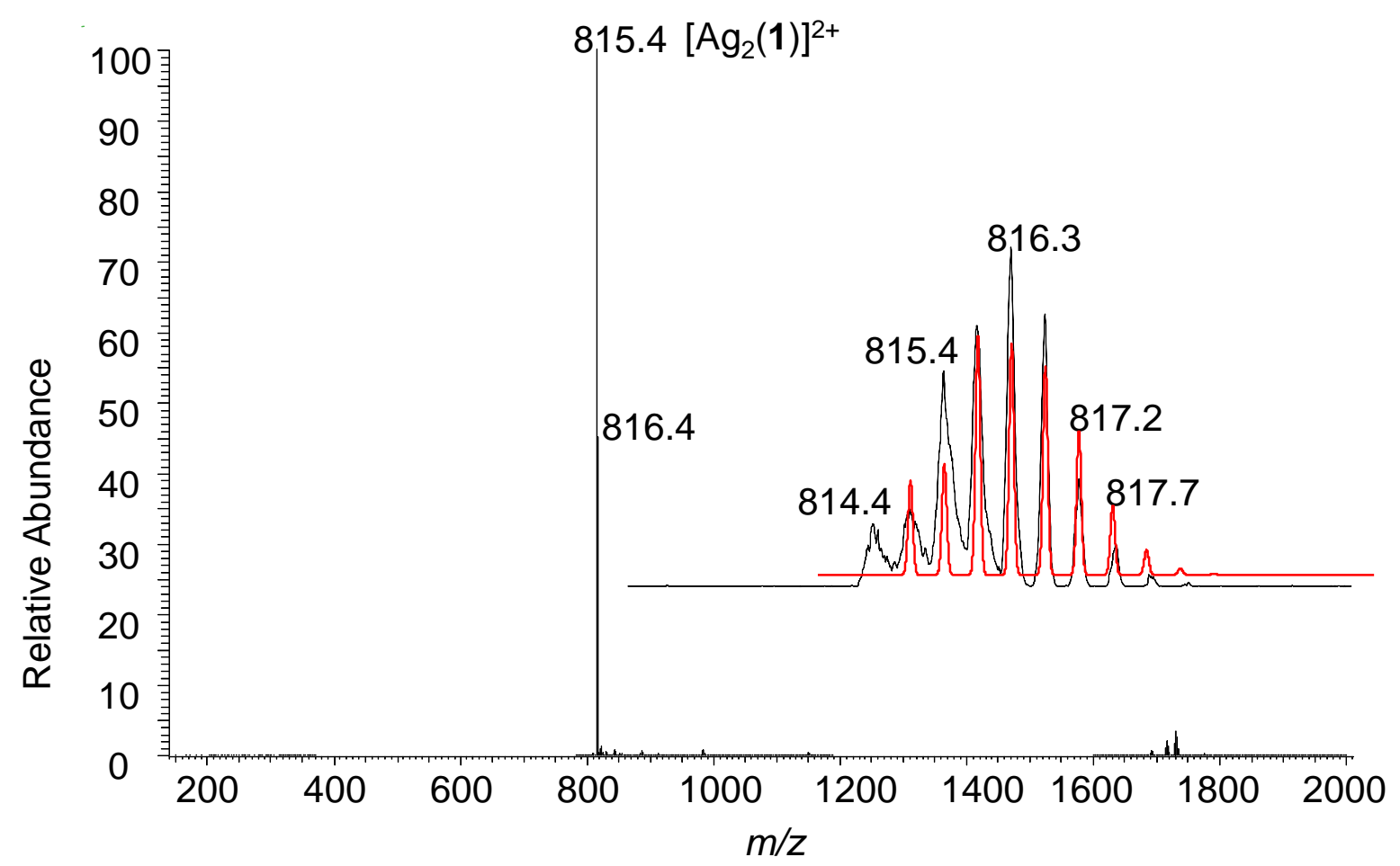

Figure S50. ESI-MS of $\left[\operatorname{Ag}_{2}(\mathbf{1})\right]^{2+}$.

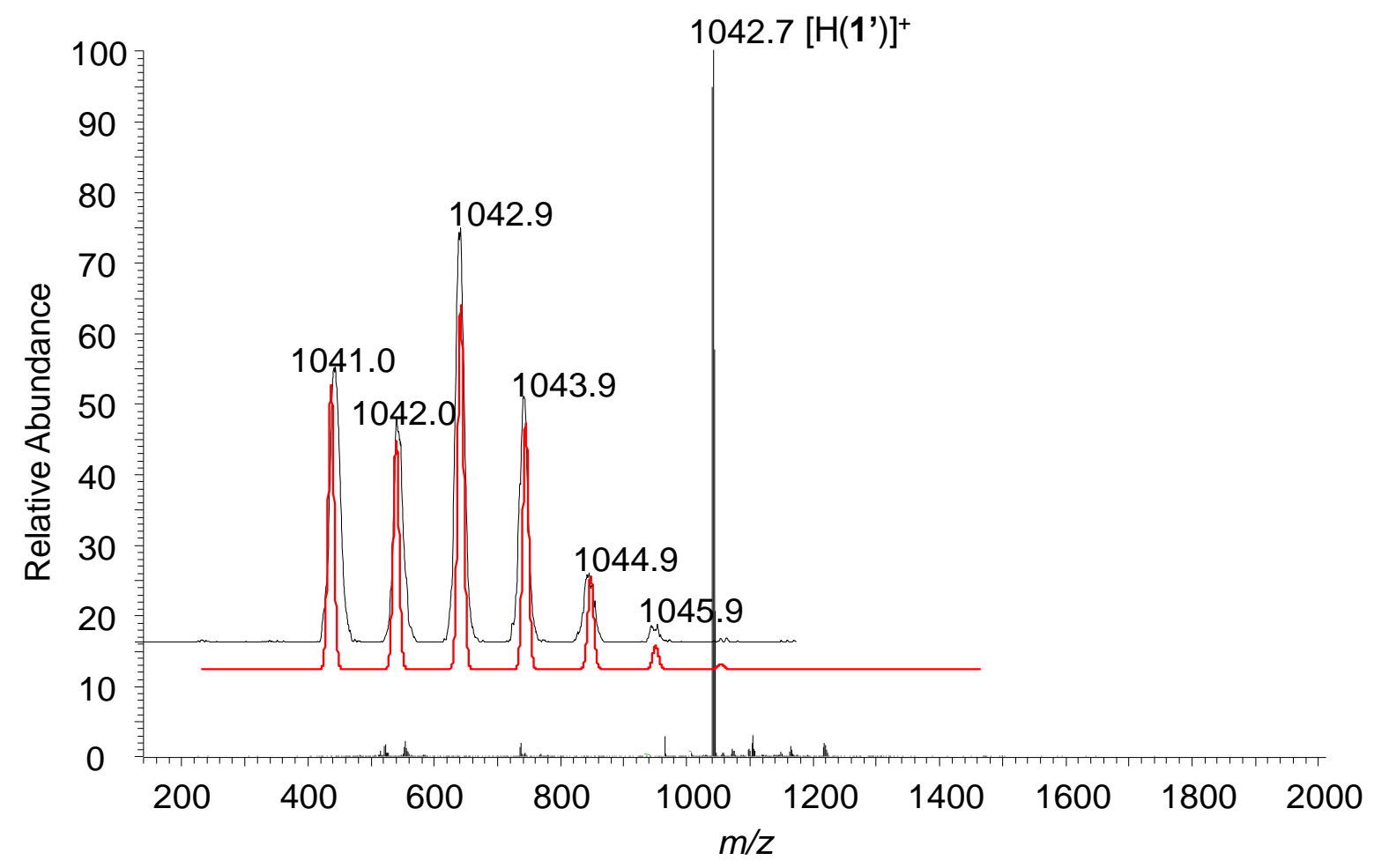

Figure S51. ESI-MS of compound 1' after protonation. 


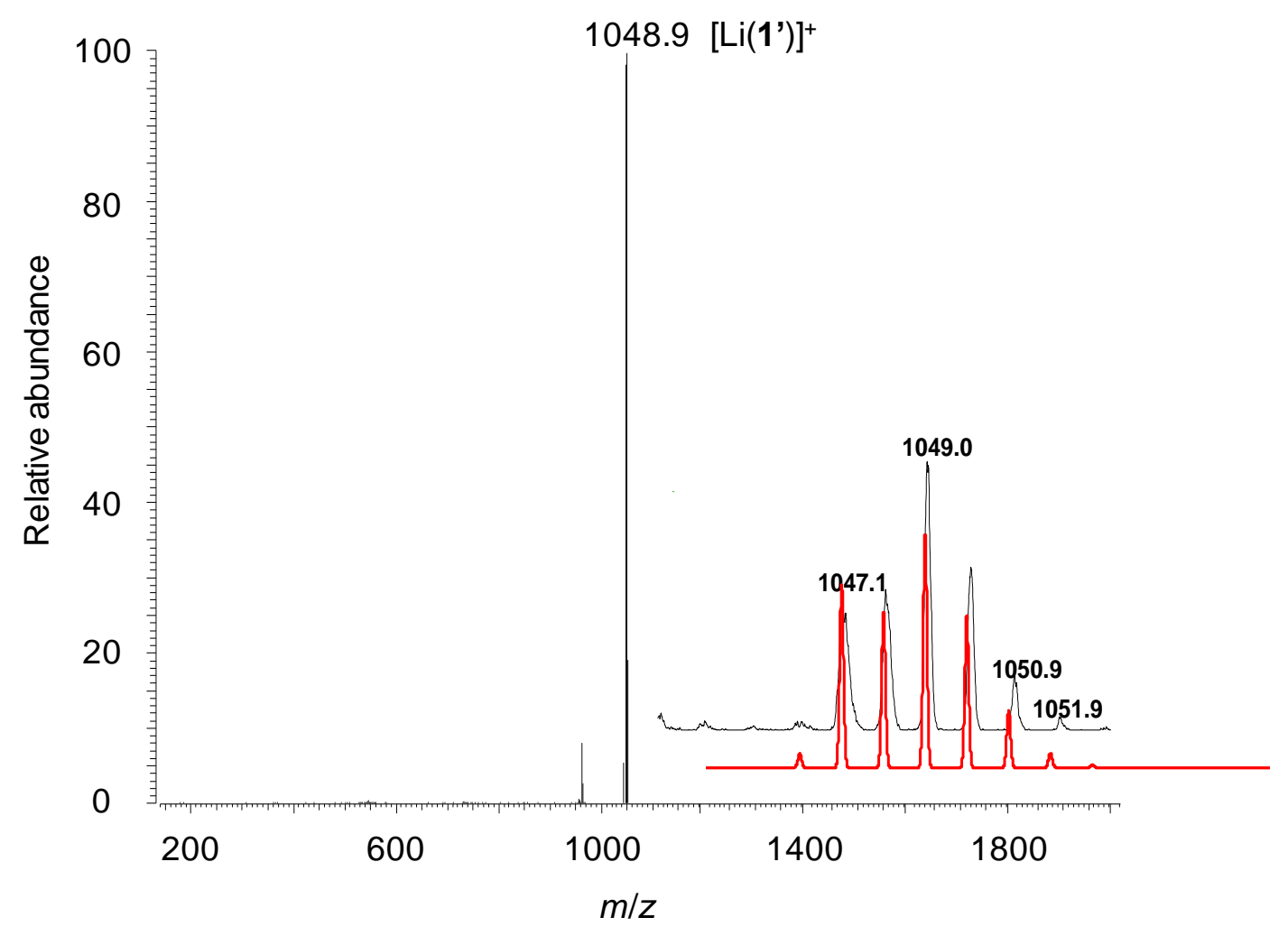

Figure S52. ESI-MS of $\left[\operatorname{Li}\left(\mathbf{1}^{\prime}\right)\right]^{+}$.

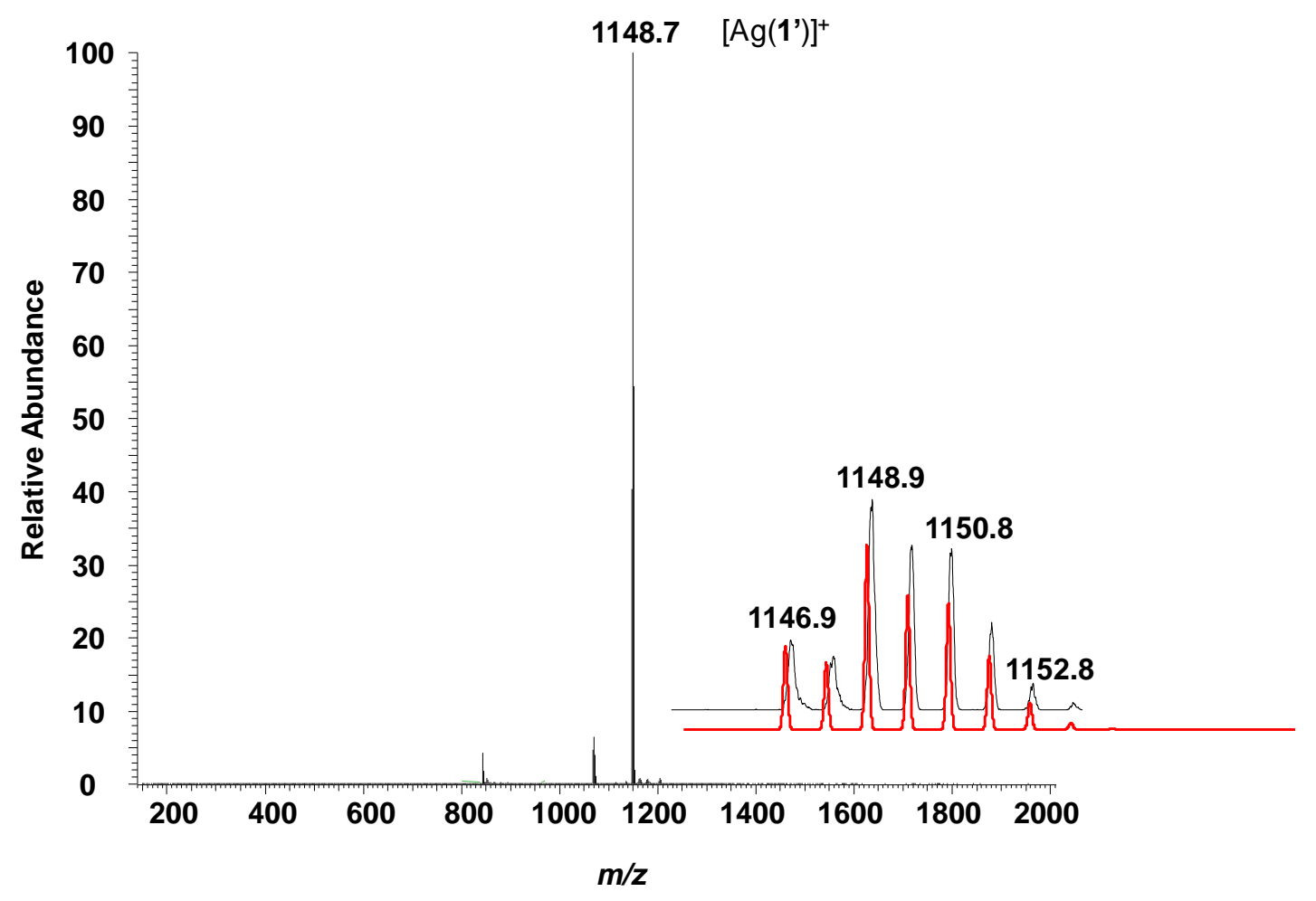

Figure S53. ESI-MS of $\left[\operatorname{Ag}\left(\mathbf{1}^{\prime}\right)\right]^{+}$. 
7. UV-Vis and fluorescence spectra

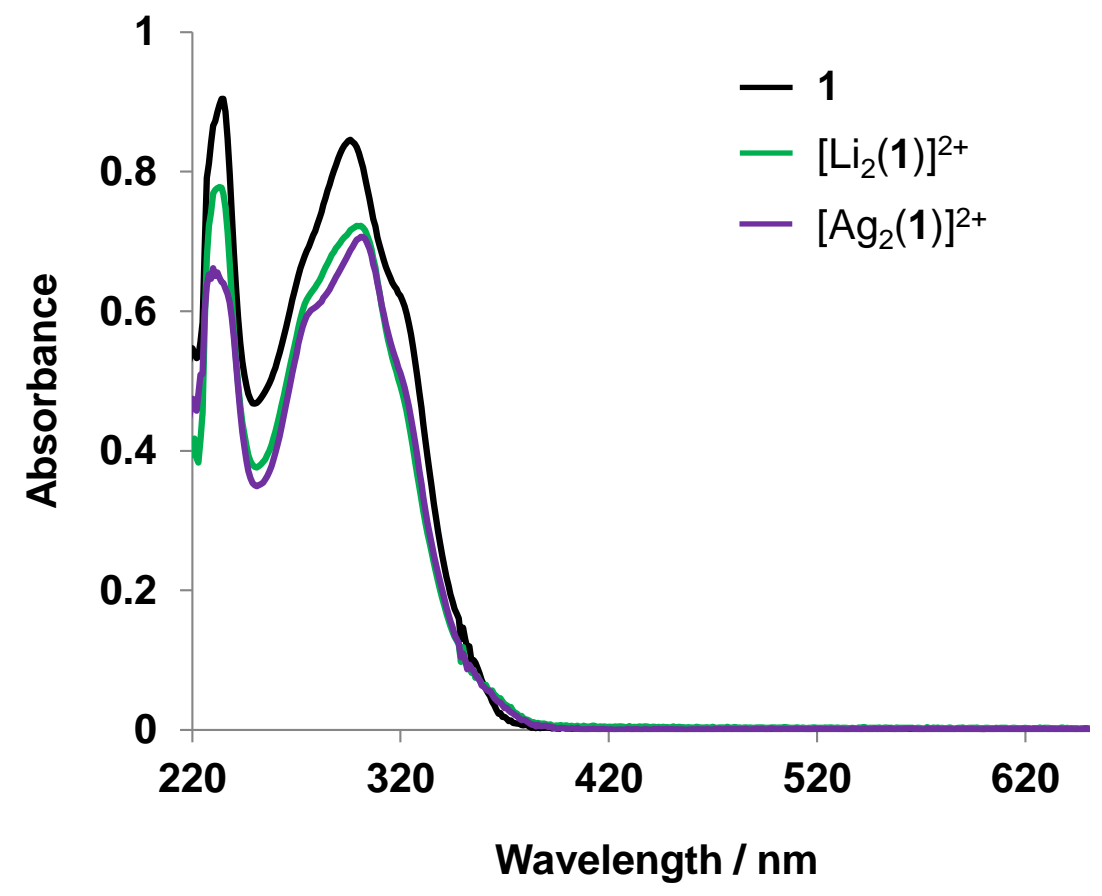

Figure S54. UV-vis spectra $\left(1.2 \times 10^{-5} \mathrm{M}\right.$ in $\left.\mathrm{CH}_{2} \mathrm{Cl}_{2}\right)$ of $\mathbf{1},\left[\mathrm{Li}_{2}(\mathbf{1})\right]^{2+}$ and $\left[\mathrm{Ag}_{2}(\mathbf{1})\right]^{2+}$ at $298 \mathrm{~K}$.

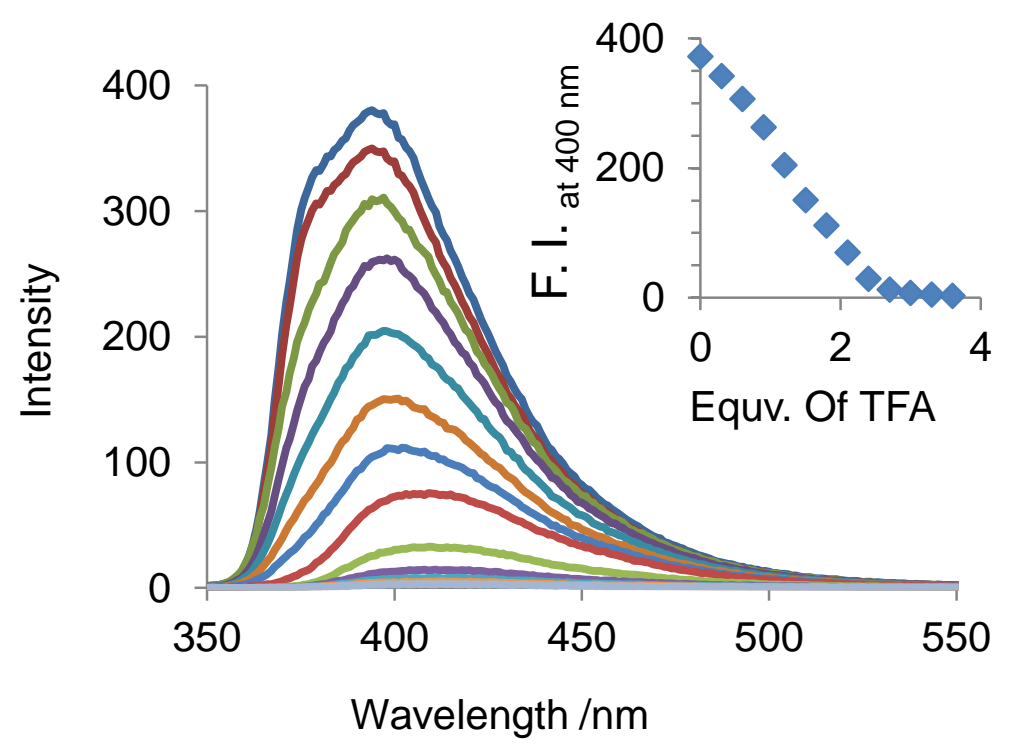

Figure S55. Fluorescence titration of rotor $1\left(1.25 \times 10^{-4} \mathrm{M}\right)$ with trifluoroacetic acid (TFA) $\left(2.0 \times 10^{-2} \mathrm{M}\right)$ in $\mathrm{CH}_{2} \mathrm{Cl}_{2}: \mathrm{CH}_{3} \mathrm{CN}(5: 1), \lambda_{\mathrm{exc}}=330 \mathrm{~nm}$. Change in fluorescence intensity at $\lambda=$ $400 \mathrm{~nm}$ (see inset). 
A)

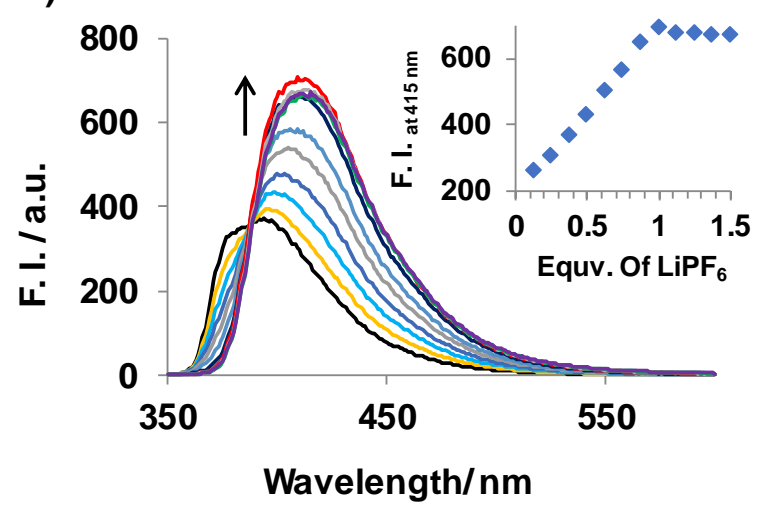

B)

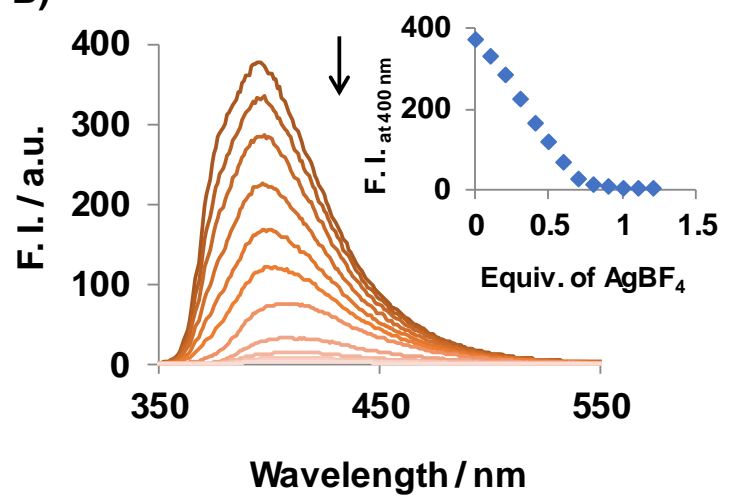

Figure S56. Fluorescence titration of rotor $1\left(1.25 \times 10^{-4} \mathrm{M}\right)$ with $(\mathrm{A}) \mathrm{LiPF}_{6}\left(1.0 \times 10^{-2} \mathrm{M}\right)$ (B) $\mathrm{AgBF}_{4}\left(1.0 \times 10^{-2} \mathrm{M}\right)$ in $\mathrm{CH}_{2} \mathrm{Cl}_{2}: \mathrm{CH}_{3} \mathrm{CN}(5: 1), \lambda_{\mathrm{exc}}=330 \mathrm{~nm}$. Change in fluorescence intensity at $\lambda=415 \mathrm{~nm}$ (for A) and $400 \mathrm{~nm}$ (for B) (see inset). In B a substantial amount of collisional quenching is involved.

Interestingly, upon addition of 1.0 equiv of $\mathrm{AgBF}_{4}$ to 1 , the single silver(I)-loading in complex $[\mathrm{Ag}(1)]^{+}$is already sufficient to fully quench the fluorescence of ligand 1 . Addition of a second equivalent of $\mathrm{AgBF}_{4}$ leads to no further change in the fluorescence intensity (see Fig. S56B). Similarly, in Fig. S56A we observe that addition of the first 1.0 equiv of $\mathrm{LiPF}_{6}$ is responsible for HETTAP complexation and for all fluorescence changes. Filling the second coordination site does not entail any further fluorescence changes. Single loading of 1 with $\mathrm{M}^{+}$leads to quantitative and selective HETTAP complexation as demonstrated by the preparation and characterization of the individual complexes $[\mathrm{Ag}(\mathbf{1})]^{+}$and $[\mathrm{Li}(\mathbf{1})]^{+}$, respectively (SI, Figs. S22 \& S23).

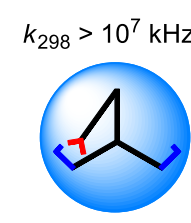

1

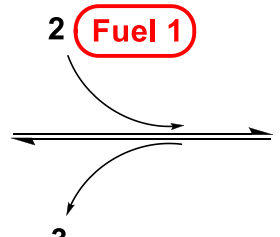

3

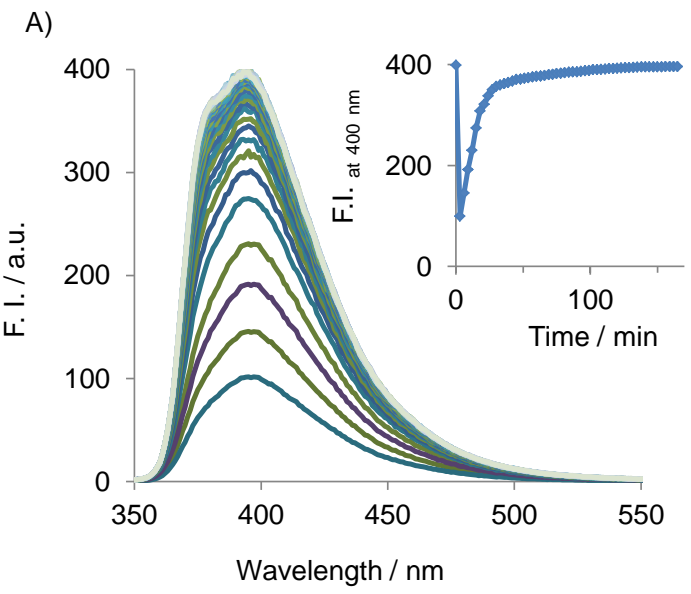

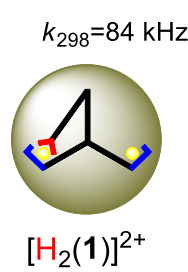

B)

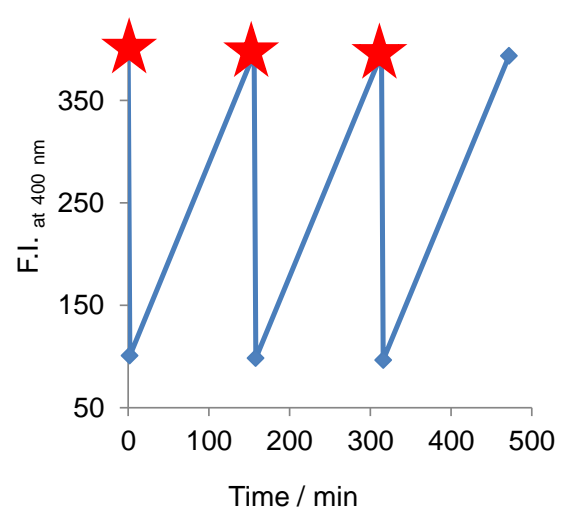

Figure S57. (A) Changes in fluorescence intensity after addition of 2.0 equiv of acid 2 to a solution of $1(150 \mu \mathrm{M})$ in $\mathrm{CH}_{2} \mathrm{Cl}_{2}\left(\lambda_{\text {exc }}=330 \mathrm{~nm}\right)$ (time dependence reported at $\lambda=400 \mathrm{~nm}$, see inset). (B) Changes in fluorescence intensity at $\lambda=400 \mathrm{~nm}$ with time for three successive cycles $\left(\lambda_{\text {exc }}=330 \mathrm{~nm}\right)$. ${ }^{*}$ Red marks indicate the repeated addition of 2.0 equiv of acid 2. 


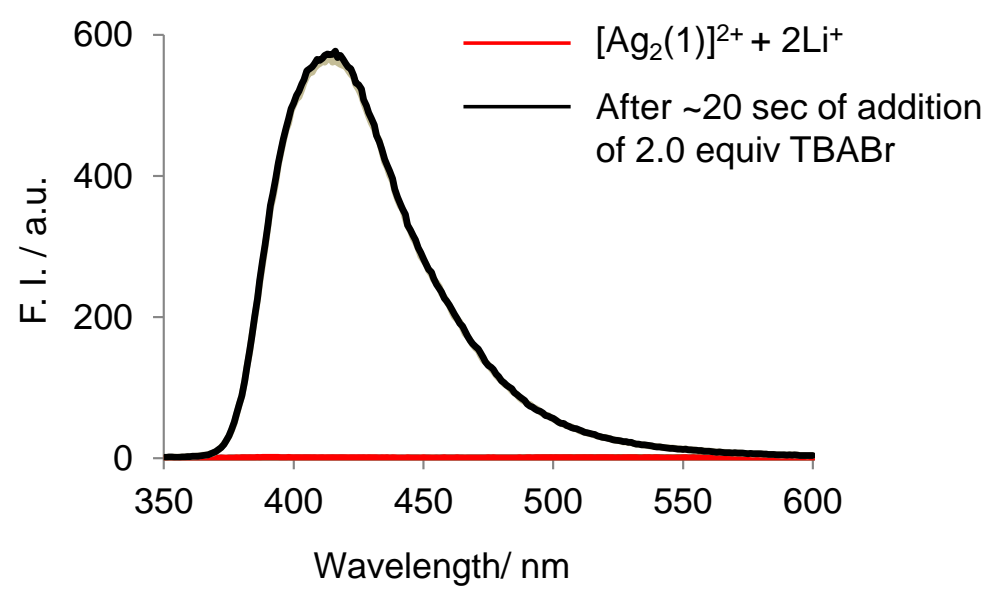

Figure S58. Addition of 2.0 equiv of $\mathrm{TBABr}$ (as solid) to the mixture of $\mathbf{1}\left(1.25 \times 10^{-4} \mathrm{M}\right.$ ), $\mathrm{Ag}^{+}, \mathrm{Li}^{+}$in 1:2:2 ratio (= mixture of $\left[\mathrm{Ag}_{2}(\mathbf{1})\right]^{2+}$ and 2.0 equiv of $\mathrm{Li}^{+}$) showing immediate increase in the emission intensity at $\lambda=415 \mathrm{~nm}$ and formation of $\left[\operatorname{Li}_{2}(\mathbf{1})\right]^{2+}\left(\lambda_{\mathrm{exc}}=330 \mathrm{~nm}\right)$.
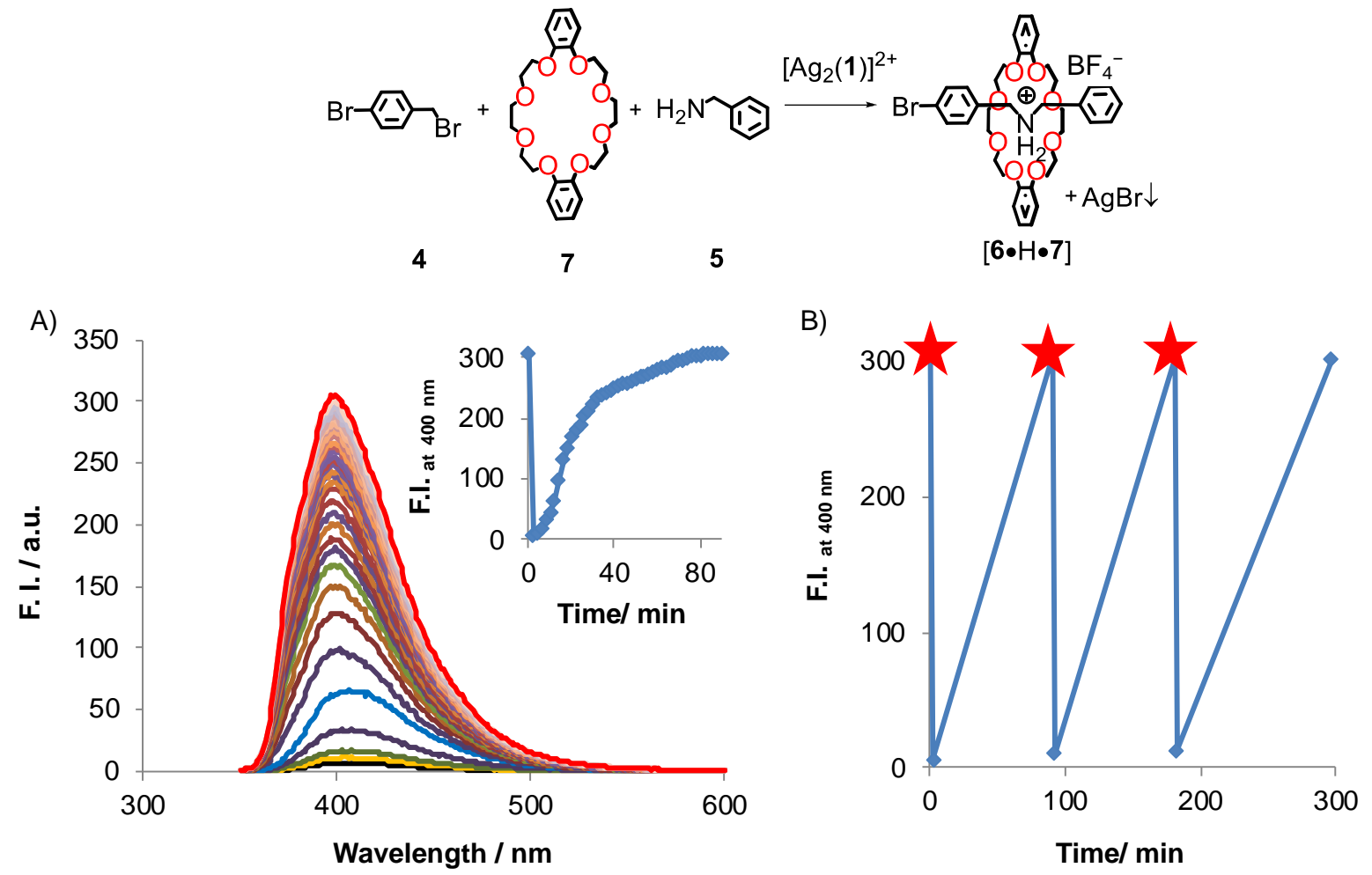

Figure S59. Changes in fluorescence intensity after addition of 2.0 equiv of $\mathrm{AgBF}_{4}$ to the mixture of $1(125 \mu \mathrm{M}), \mathbf{4}(\sim 4.0 \mathrm{mM}), \mathbf{5}(\sim 4.0 \mathrm{mM})$ and $7(\sim 4.0 \mathrm{mM})$ in $\mathrm{CH}_{2} \mathrm{Cl}_{2} / \mathrm{CH}_{3} \mathrm{CN}(5: 1)$ $\left(\lambda_{\mathrm{exc}}=330 \mathrm{~nm}\right)$. Changes in fluorescence intensity at $\lambda=400 \mathrm{~nm}\left(\lambda_{\mathrm{exc}}=330 \mathrm{~nm}\right)$ with time (see inset). * Red marks indicate the repeated addition of 2.0 equiv of $\mathrm{AgBF}_{4}$. 

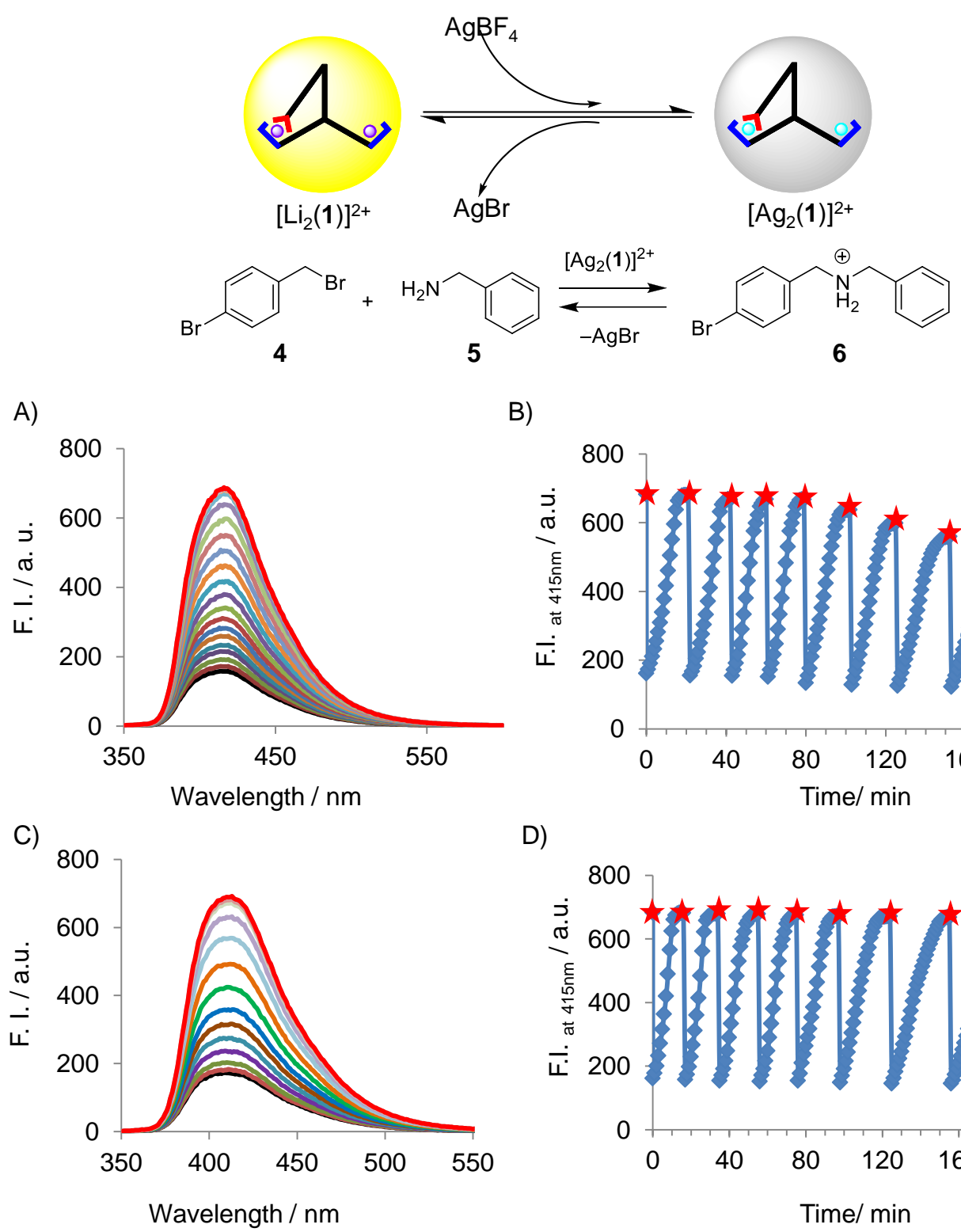

B)

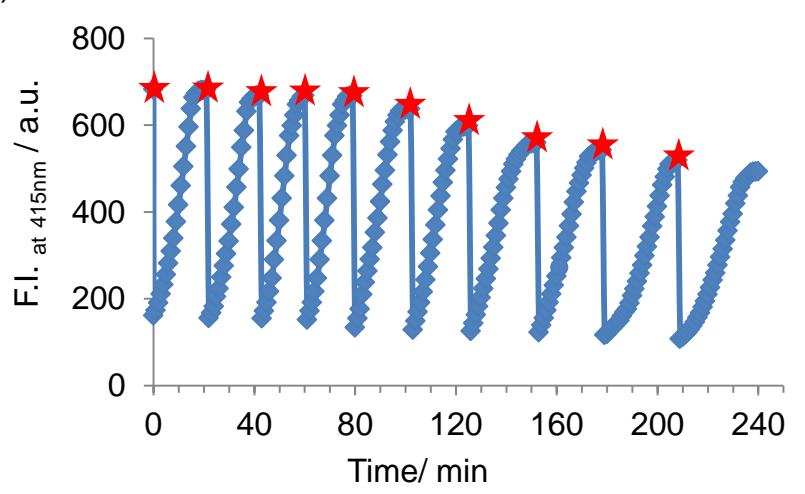

D)

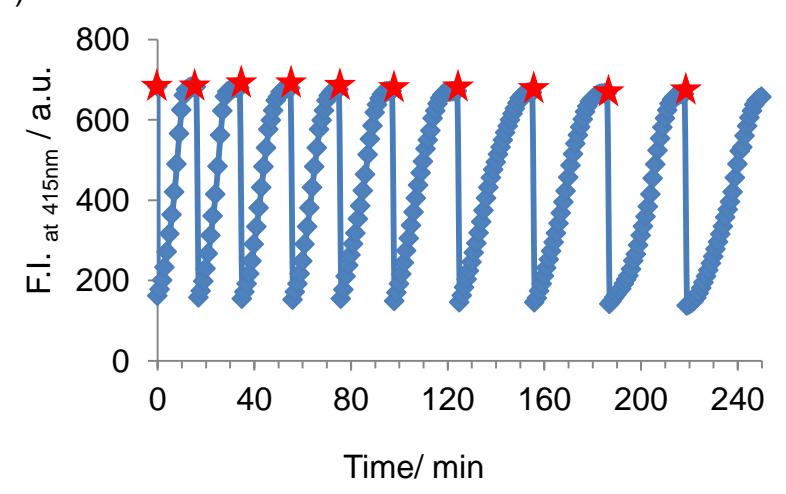

Figure S60. Changes in fluorescence intensity after addition of 2.0 equiv of $\mathrm{AgBF}_{4}$ to the mixture of $\left[\mathrm{Li}_{2}(\mathbf{1})\right]^{2+}(125 \mu \mathrm{M}), 4(\sim 4.0 \mathrm{mM})$ and $\mathbf{5}(\sim 4.0 \mathrm{mM})$ in $\mathrm{CH}_{2} \mathrm{Cl}_{2} / \mathrm{CH}_{3} \mathrm{CN}$ (5:1) $\left(\lambda_{\mathrm{exc}}=\right.$ $330 \mathrm{~nm}$ ). (B) Changes in fluorescence intensity at $\lambda=415 \mathrm{~nm}$ with time for ten successive cycles $\left(\lambda_{\text {exc }}=330 \mathrm{~nm}\right)$. (C) Changes in fluorescence intensity after addition of 2.0 equiv of $\mathrm{AgBF}_{4}$ to the mixture of $\left[\operatorname{Li}_{2}(\mathbf{1})\right]^{2+}(125 \mu \mathrm{M}), 4(\sim 4.0 \mathrm{mM}), \mathbf{5}(\sim 4.0 \mathrm{mM})$ and $7(\sim 4 \mathrm{mM})$ in $\mathrm{CH}_{2} \mathrm{Cl}_{2} / \mathrm{CH}_{3} \mathrm{CN}(5: 1)\left(\lambda_{\mathrm{exc}}=330 \mathrm{~nm}\right)$. (D) Changes in fluorescence intensity in the presence of crown 7 at $\lambda=415 \mathrm{~nm}$ for ten cycles with time after. ${ }^{*}$ Red marks indicate the repeated addition of 2.0 equiv of $\mathrm{AgBF}_{4}$. 

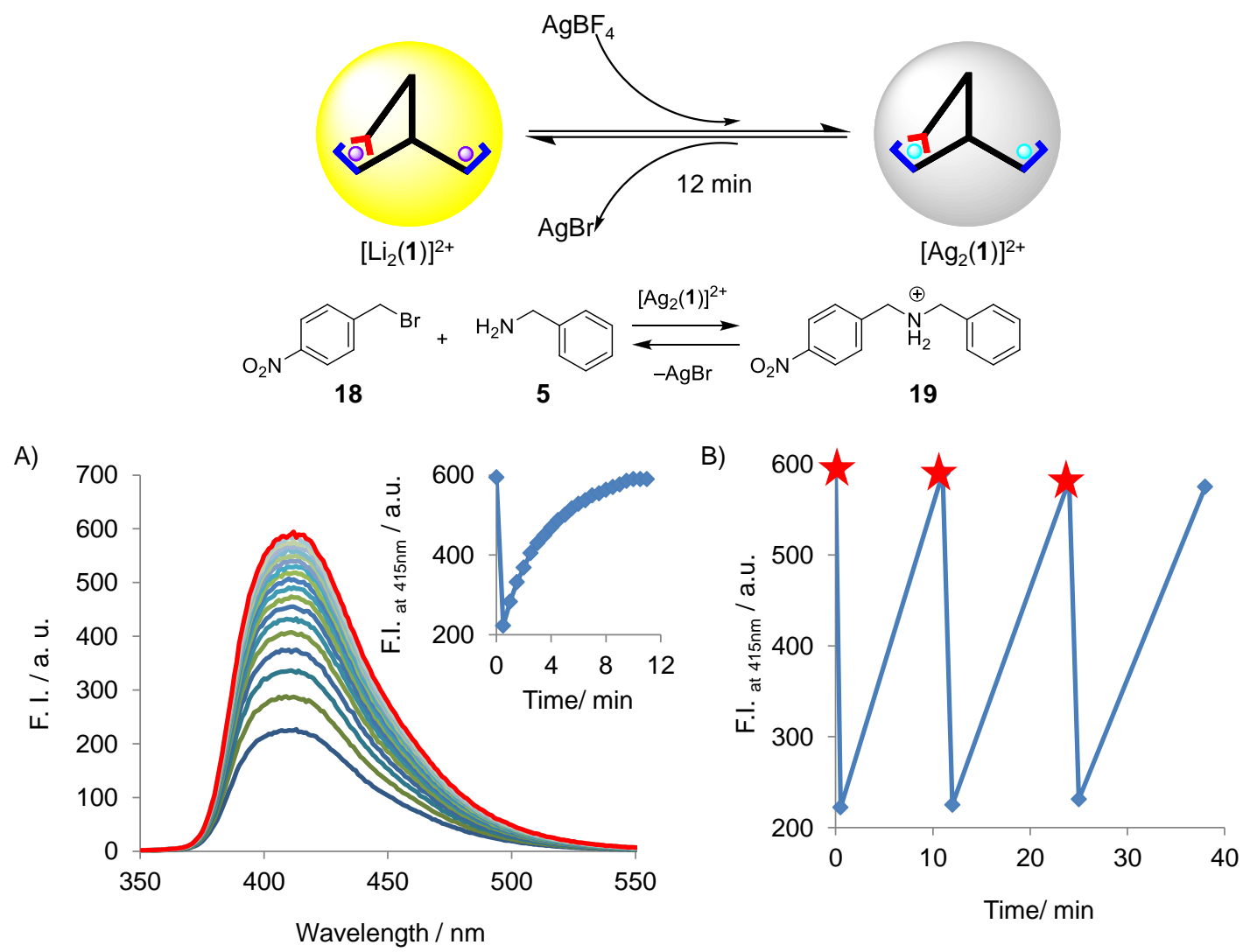

Figure S61. Changes in fluorescence intensity after addition of 2.0 equiv of $\mathrm{AgBF}_{4}$ to the mixture of $\left[\mathrm{Li}_{2}(\mathbf{1})\right]^{2+}(125 \mu \mathrm{M}), \mathbf{1 8}(\sim 4.0 \mathrm{mM})$ and $\mathbf{5}(\sim 4.0 \mathrm{mM})$ in $\mathrm{CH}_{2} \mathrm{Cl}_{2}: \mathrm{CH}_{3} \mathrm{CN}(5: 1)\left(\lambda_{\text {exc }}\right.$ $=330 \mathrm{~nm}$ ). (B) Changes in fluorescence intensity at $\lambda=415 \mathrm{~nm}$ with time for three successive cycles $\left(\lambda_{\mathrm{exc}}=330 \mathrm{~nm}\right)$. 


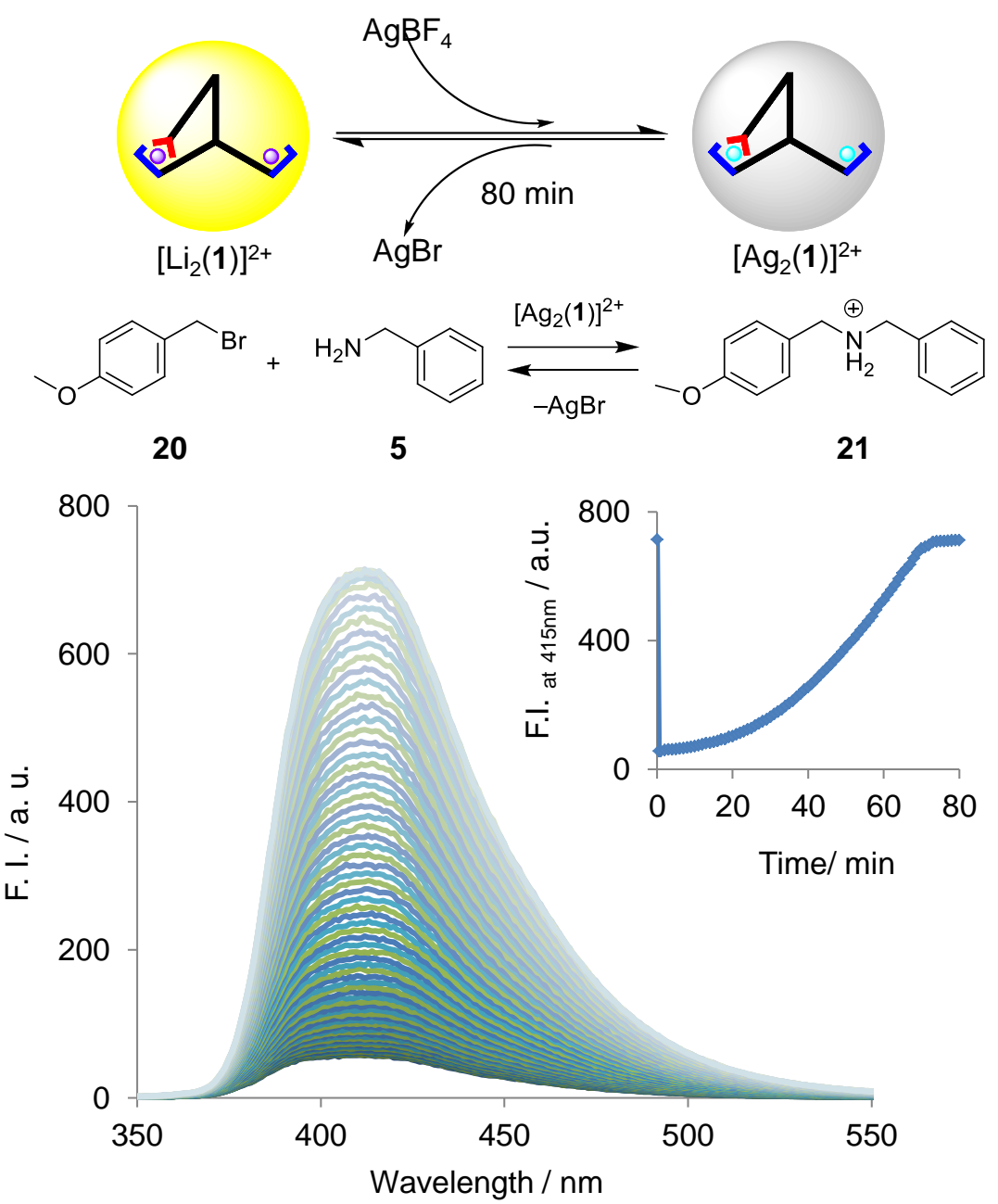

Figure S62. Change in fluorescence intensity after addition of 2.0 equiv of $\mathrm{AgBF}_{4}$ to the mixture of $\left[\mathrm{Li}_{2}(\mathbf{1})\right]^{2+}(125 \mu \mathrm{M}), \mathbf{2 0}(\sim 4.0 \mathrm{mM})$ and $\mathbf{5}(\sim 4.0 \mathrm{mM})$ in $\mathrm{CH}_{2} \mathrm{Cl}_{2}: \mathrm{CH}_{3} \mathrm{CN}(5: 1)\left(\lambda_{\text {exc }}\right.$ $=330 \mathrm{~nm})$. Changes in fluorescence intensity at $\lambda=415 \mathrm{~nm}\left(\lambda_{\mathrm{exc}}=330 \mathrm{~nm}\right)$ with time (see inset). 

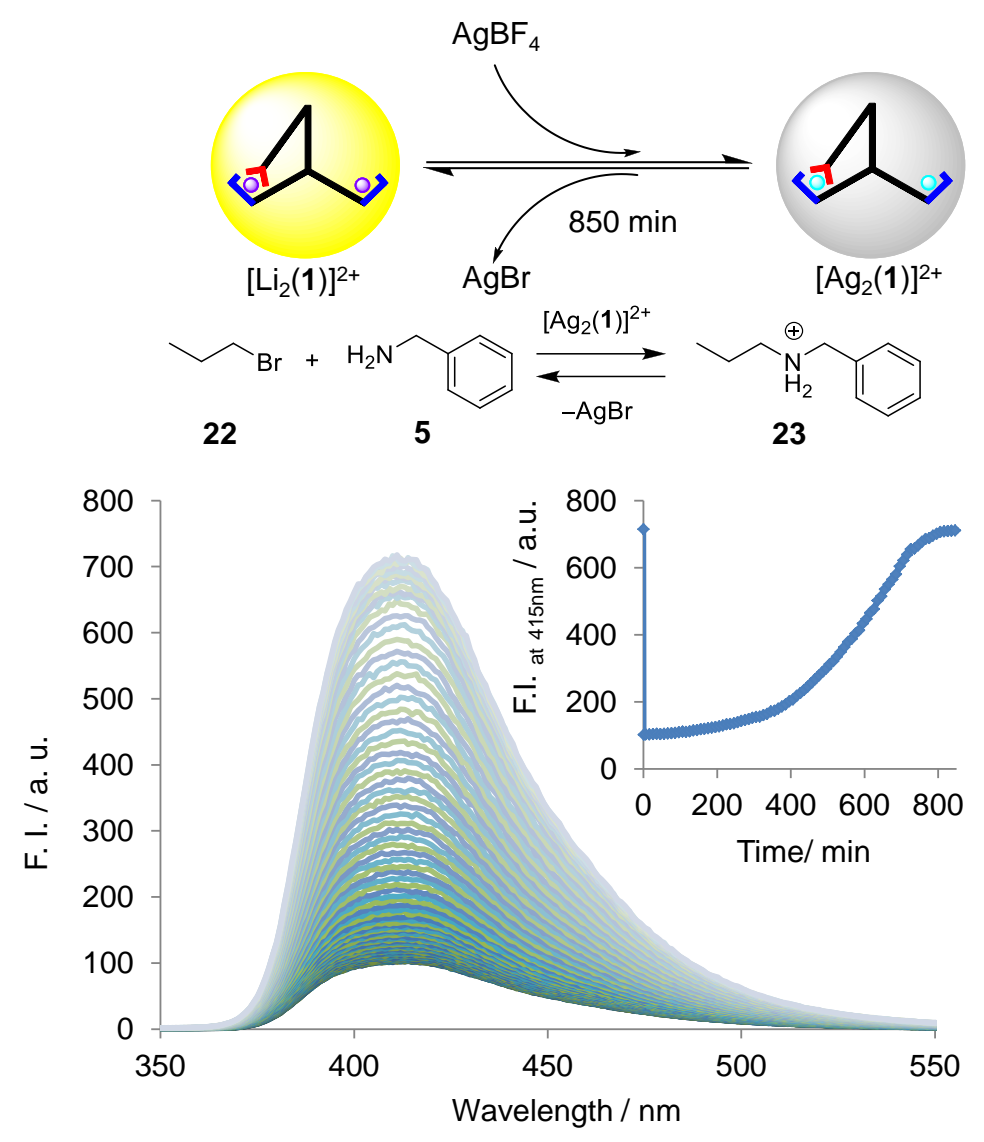

Figure S63. Changes in fluorescence intensity after addition of 2.0 equiv of $\mathrm{AgBF}_{4}$ to the mixture of $\left[\mathrm{Li}_{2}(\mathbf{1})\right]^{2+}(125 \mu \mathrm{M}), 22(\sim 4.0 \mathrm{mM})$ and $\mathbf{5}(\sim 4.0 \mathrm{mM})$ in $\mathrm{CH}_{2} \mathrm{Cl}_{2} / \mathrm{CH}_{3} \mathrm{CN}$ (5:1) $\left(\lambda_{\text {exc }}\right.$ $=330 \mathrm{~nm})$. Changes in fluorescence intensity at $\lambda=415 \mathrm{~nm}\left(\lambda_{\mathrm{exc}}=330 \mathrm{~nm}\right)$ with time (see inset).

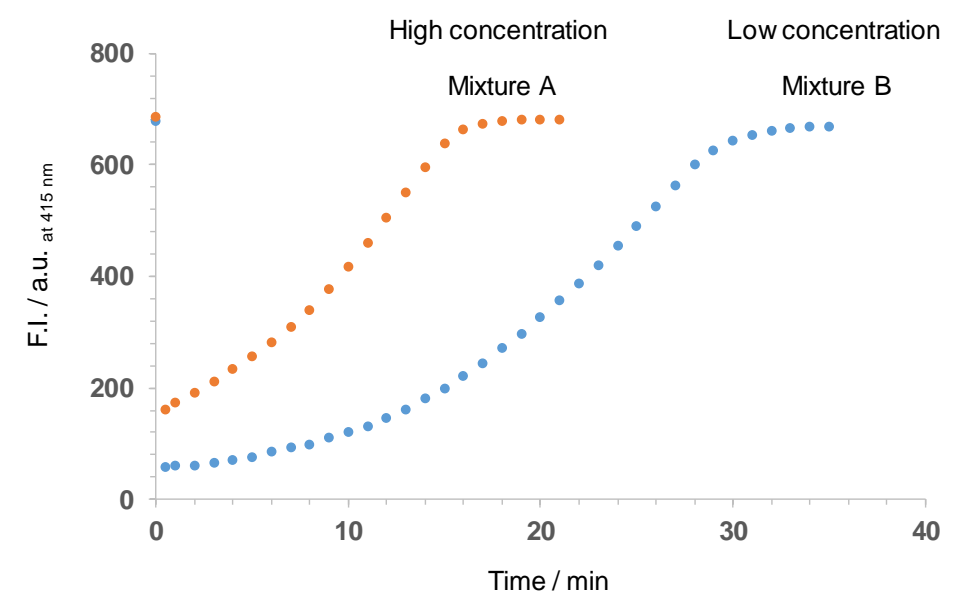

Figure S64. Duration of the silver(I) fueling cycle depends on the concentration of the substrates. Changes in fluorescence intensity at $\lambda=415 \mathrm{~nm}$ with time after addition of 2.0 equiv of $\mathrm{AgBF}_{4}$ to the mixture $\mathrm{A}$ or $\mathrm{B}$ in $\mathrm{CH}_{2} \mathrm{Cl}_{2} / \mathrm{CH}_{3} \mathrm{CN}(5: 1)\left(\lambda_{\mathrm{exc}}=330 \mathrm{~nm}\right)$. Mixture $\mathrm{A}$ : rotor $\left[\operatorname{Li}_{2}(\mathbf{1})\right]^{2+}(125 \mu \mathrm{M}), 4(\sim 4.0 \mathrm{mM})$ and $\mathbf{5}(\sim 4.0 \mathrm{mM})$. Mixture B: rotor $\left[\operatorname{Li}_{2}(\mathbf{1})\right]^{2+}(125$ $\mu \mathrm{M}), \mathbf{4}(\sim 0.8 \mathrm{mM})$ and $\mathbf{5}(\sim 0.8 \mathrm{mM})$. 


\section{Binding constant measurements}

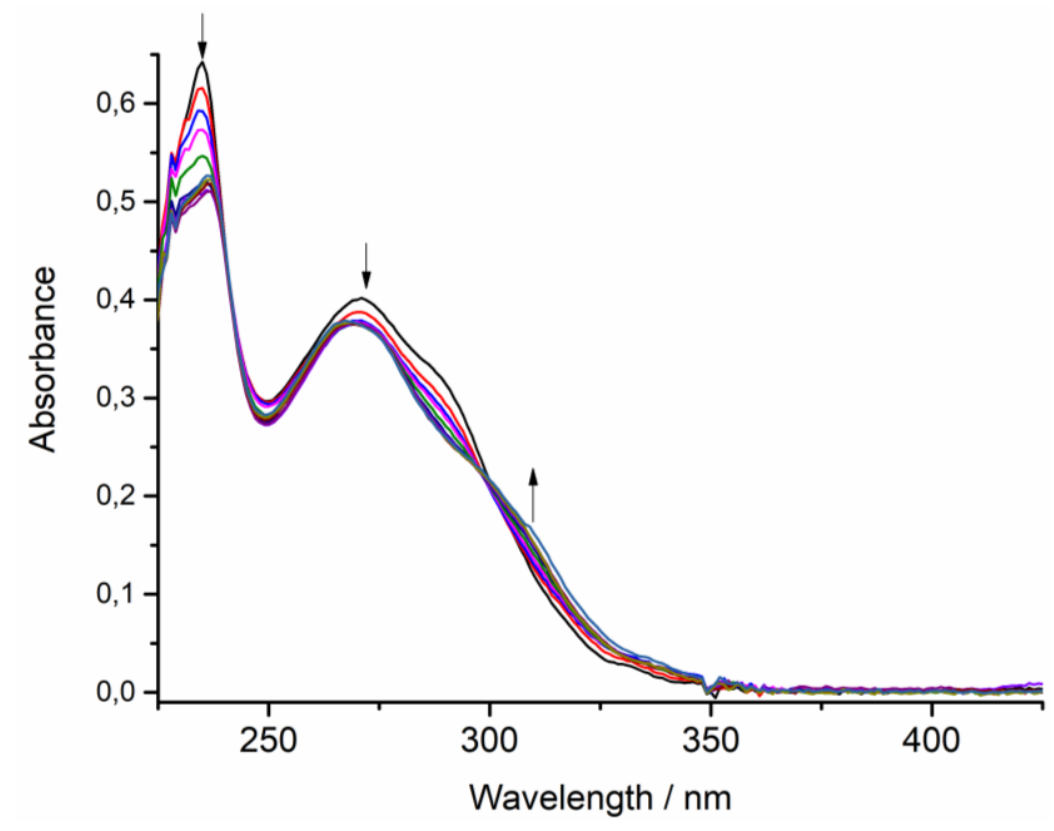

Figure S65. UV-vis titration of 2,9-dimesityl-1,10-phenanthroline $\left(\mathbf{2 4}, 1.20 \times 10^{-5} \mathrm{M}\right)$ vs. $\mathrm{AgBF}_{4}\left(2.05 \times 10^{-3} \mathrm{M}\right)$ in $\mathrm{CH}_{2} \mathrm{Cl}_{2}: \mathrm{CH}_{3} \mathrm{CN}(5: 1)$ at $298 \mathrm{~K}$. Binding constant was determined as $\log K=5.14 \pm 0.16$ using SPECFIT $^{\circledR}$ software.

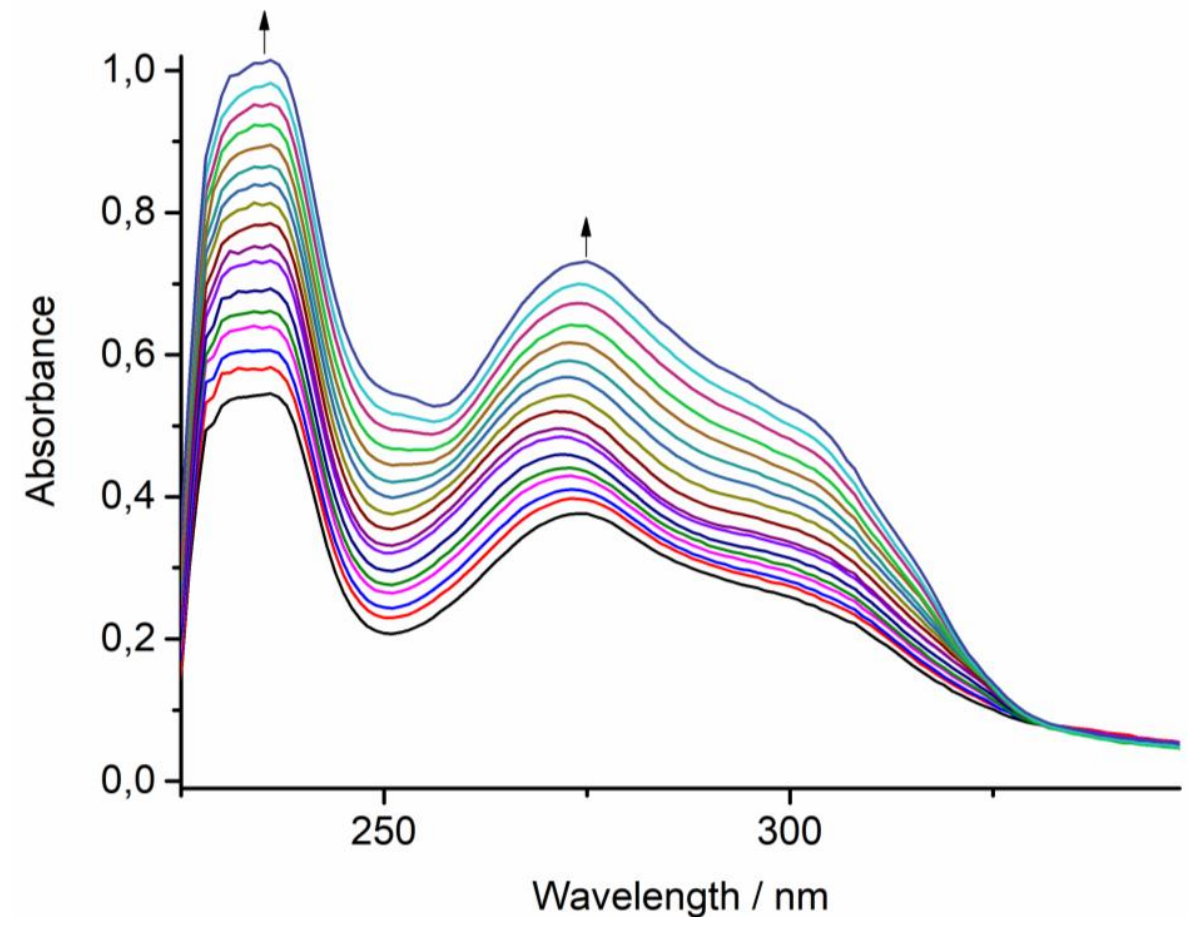

Figure S66. UV-vis titration of $[\mathrm{Ag}(\mathbf{2 4})]^{+}\left(8.64 \times 10^{-6} \mathrm{M}\right)$ vs. 2,2':6',2"'-terpyridine $(\mathbf{2 5}, 1.87$ $\left.\times 10^{-4} \mathrm{M}\right)$ in $\mathrm{CH}_{2} \mathrm{Cl}_{2}: \mathrm{CH}_{3} \mathrm{CN}(5: 1)$ at $298 \mathrm{~K}$. Binding constant was determined as $\log \mathrm{K}=$ $3.97 \pm 0.25$ using SPECFIT ${ }^{\circledR}$ software. 


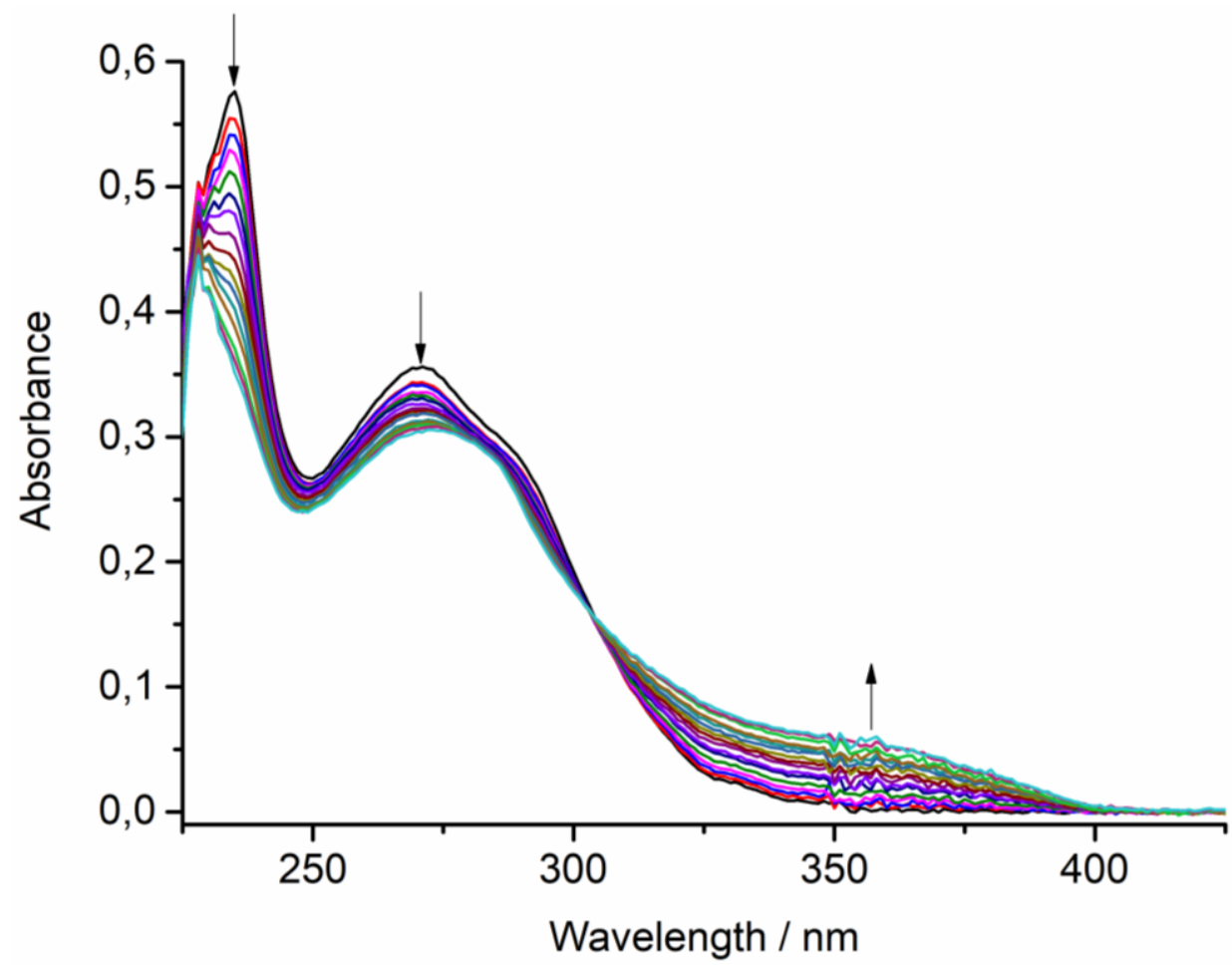

Figure S67. UV-vis titration of 2,9-dimesityl-1,10-phenanthroline $\left(\mathbf{2 4}, 1.20 \times 10^{-5} \mathrm{M}\right)$ vs. $\mathrm{LiPF}_{6}\left(3.03 \times 10^{-3} \mathrm{M}\right)$ in $\mathrm{CH}_{2} \mathrm{Cl}_{2}: \mathrm{CH}_{3} \mathrm{CN}(5: 1)$ at $298 \mathrm{~K}$. Binding constant was determined as $\log K=4.68 \pm 0.42$ using SPECFIT $^{\circledR}$ software.

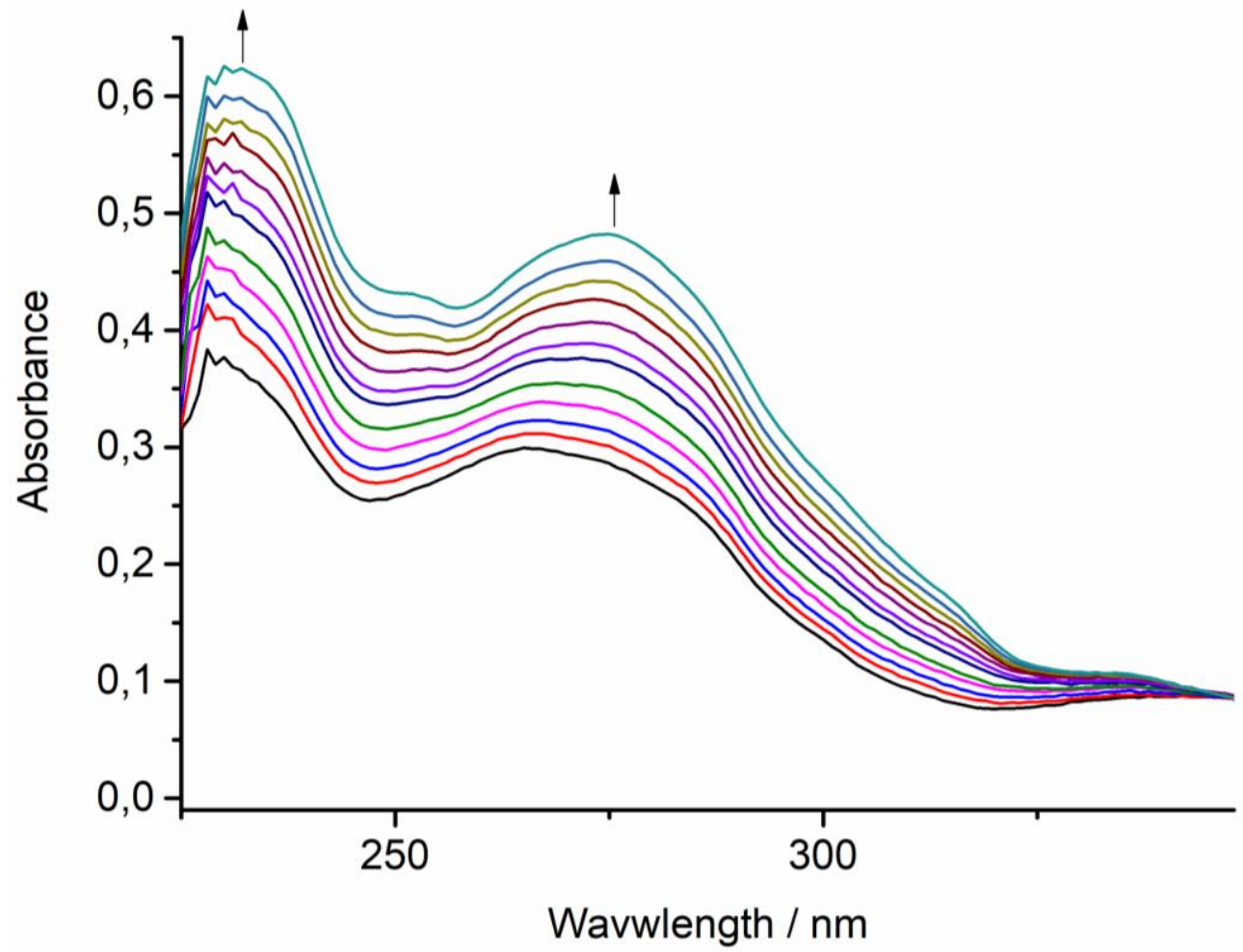

Figure 68. UV-vis titration of $[\mathrm{Li}(\mathbf{2 4})]^{+}\left(1.20 \times 10^{-5} \mathrm{M}\right)$ vs. 2,2':6', 2"'-terpyridine $(\mathbf{2 5}, 1.03 \times$ $\left.10^{-3} \mathrm{M}\right)$ in $\mathrm{CH}_{2} \mathrm{Cl}_{2}: \mathrm{CH}_{3} \mathrm{CN}(5: 1)$ at $298 \mathrm{~K}$. Binding constant was determined as $\log K=3.01$ \pm 0.07 using SPECFIT $^{\circledR}$ software. 
Table S1. Binding constants in model complexes

\begin{tabular}{|c|c|}
\hline Model Complex & Binding constant $(\log K)$ \\
\hline $\mathrm{Ag}^{+}+\mathbf{2 4} \rightarrow[\mathrm{Ag}(\mathbf{2 4})]^{+}$ & $5.14 \pm 0.16$ \\
\hline$\left.[\mathrm{Ag}(\mathbf{2 4})]^{+}+\mathbf{2 5} \rightarrow \operatorname{Ag}(\mathbf{2 4})(\mathbf{2 5})\right]^{+}$ & $3.97 \pm 0.25$ \\
\hline $\mathrm{Li}^{+}+\mathbf{2 4} \rightarrow[\mathrm{Li}(\mathbf{2 4})]^{+}$ & $4.68 \pm 0.42$ \\
\hline$[\mathrm{Li}(\mathbf{2 4})]^{+}+\mathbf{2 5} \rightarrow[\operatorname{Li}(\mathbf{2 4})(\mathbf{2 5})]^{+}$ & $3.01 \pm 0.07$ \\
\hline$\left[\operatorname{Ag}(\mathbf{2 5})_{2}\right]^{+}(\operatorname{ref} 7)$ & $6.78 \pm 0.25$ \\
\hline$\left[\operatorname{Li}(\mathbf{2 5})_{2}\right]^{+}(\operatorname{ref} 8)$ & $8.20 \pm 0.30$ \\
\hline
\end{tabular}

Compound 24 = 2,9-dimesityl-1,10-phenanthroline

Compound $25=2,2$ ':6',2”-terpyridine

\section{References}

1. Goswami, A.; Paul, I.; Schmittel, M. Chem. Commun. 2017, 53, 5186-5189.

2. Álvarez-Perez, A.; González-Rodríguez, C.; García-Yebra, C.; Varela, J. A.; Oñate, E.;

Esteruelas, M. A.; Saá, C. Angew. Chem., Int. Ed. 2015, 54, 13357-13361.

3. Goswami, A.; Gaikwad, S.; Schmittel, M. Chem. Eur. J. 10.1002/chem.202004416

4. Samanta, S. K.; Schmittel, M. J. Am. Chem. Soc. 2013, 135,18794-18797.

5. Ashton, P. R.; Ballardini, R.; Balzani, V.; Gomez-Lopez, M.; Lawrence, S. E.; Martinez-

Diaz, M. V.; Montalti, M.; Piersanti, A.; Prodi, L.; Stoddart J. F. and Williams, D. J. J. Am.

Chem. Soc. 1997, 119, 10641-10651.

6. NMR Spectrum Calculations: WinDNMR, Version 7.1.13. Hans J. Reich, Department of Chemistry, University of Wisconsin.

7. Paul, I.; Mittal, N.; De, S.; Bolte M. and Schmittel, M. J. Am. Chem. Soc. 2019, 141, 5139-5143.

8. Ghosh, A.; Paul, I. and Schmittel M. J. Am. Chem. Soc. 2019, 141, 18954-18957. 